\title{
Financial Bubble Implosion and Reverse Regression*
}

\author{
Peter C. B. Phillips \\ Yale University, University of Auckland, \\ University of Southampton \&5 Singapore Management University \\ Shu-Ping Shi \\ Macquarie University
}

March 15, 2017

\begin{abstract}
Expansion and collapse are two key features of a financial asset bubble. Bubble expansion may be modeled using a mildly explosive process. Bubble implosion may take several different forms depending on the nature of the collapse and therefore requires some flexibility in modeling. This paper first strengthens the theoretical foundation of the real time bubble monitoring strategy proposed in Phillips, Shi and Yu (2015a,b, PSY) by developing analytics and studying the performance characteristics of the testing algorithm under alternative forms of bubble implosion which capture various return paths to market normalcy. Second, we propose a new reverse sample use of the PSY procedure for detecting crises and estimating the date of market recovery. Consistency of the dating estimators is established and the limit theory addresses new complications arising from the alternative forms of bubble implosion and the endogeneity effects present in the reverse regression. A real-time version of the strategy is provided that is suited for practical implementation. Simulations explore the finite sample performance of the strategy for dating market recovery. The use of the PSY strategy for bubble monitoring and the new procedure for crisis detection are illustrated with an application to the Nasdaq stock market.
\end{abstract}

Keywords: Bubble implosion, Dating algorithm, Limit theory, Market recovery, Nasdaq market.

JEL classification: C15, C22

\section{Introduction}

Following the global financial crisis (GFC) there has been widespread recognition of the harm that financial bubbles can inflict on real economies. The slow recovery from the great recession in the USA and the continuing debt crisis in Europe has alerted central bankers and regulators to the

${ }^{*}$ Phillips acknowledges research support from the NSF under Grant No. SES 12-58258. Peter C.B. Phillips email: peter.phillips@yale.edu. Shuping Shi, email: shuping.shi@mq.edu.au. 
need for greater understanding of the mechanisms by which financial bubbles form, the dynamics of their evolution and collapse, and the process of contagion through which other markets and the real economy are affected. Few national economies have been unaffected by the fallout from the GFC. The hazards have therefore become a matter of considerable concern to central banks and policy makers. ${ }^{1}{ }^{2}$

While the potentially damaging impact of financial bubbles on the real economy is widely acknowledged, policy makers face major difficulties in designing corrective measures and timing their implementation. Recent econometric work has assisted the design and timing of policy measures by providing empirical techniques that detect mildly explosive bubble-like behaviour in asset prices. While some economists think it is impossible to see bubbles in their inflationary phase (Cooper, 2008), recent developments in the econometric bubble literature deliver real-time monitoring strategies, such as recursive right-sided unit root testing procedures (Phillips, Wu and Yu, 2011, PWY; Phillips, Shi and Yu, 2014; PSY), CUSUM monitoring techniques (Homm and Breitung, 2012; HB), and double-recursive algorithms (Phillips, Shi and Yu, 2015a,b) that enable bubble detection and consistent estimation of the origination and termination dates of bubble expansion.

The present work focuses on the real-time monitoring procedure of PSY, which is an extended version of the PWY recursive testing approach. The PSY algorithm has been applied to a wide range of markets, including foreign exchange, real estate, commodities and financial assets, and has attracted attention from policy makers and the financial press. ${ }^{3}$ The algorithm has been shown (PSY, 2015a,b; HM, 2012) to have superior real time monitoring and detection performance than other methods but nonetheless suffers from delay bias in detection.

The present paper contributes in two ways to this literature. First, it strengthens the foundation of the PSY approach to bubble monitoring by exploring its asymptotics and behavioral characteristics under alternative collapse scenarios which enable more flexible modeling of bubble implosion. Second, it proposes an alternative 'reverse regression' implementation strategy for detecting bubble implosion and estimating the origination and termination dates of bubble implosion. It is noted that while events of bubble implosion are often referred to as crises, crises are not necessarily led by bubbles. Since the focus of this paper is on bubble-led crises, we use the term "bubble implosion/collapse" and "crisis" interchangeably. The new algorithm, which is based on recursive reverse-sample regression, assists in reducing the delay bias of the PSY procedure in the detection of the bubble collapse date and by providing additional information on the market recovery date.

The PSY double recursion strategy is particularly designed for detection purposes when there are periodically collapsing bubbles (Blanchard, 1979) in the data. Its asymptotic and finite sample performance has been studied under several different bubble generating processes and

\footnotetext{
${ }^{1}$ Federal Reserve policymakers should deepen their understanding about how to combat speculative bubbles to reduce the chances of another financial crisis. -Donald Kohn, Former Vice Chairman of the Federal Reserve Board, March 2010.

${ }^{2}$ How do we know when irrational exuberance has unduly escalated asset values? - Alan Greenspan, Formal Chairman of Federal Reserve, December 1996 .

${ }^{3}$ See, for example, Bohl et al. (2013), Etienne et al. (2015), Chen and Funke (2013), Meyer (2013), Gutierrez (2013), and Yiu et al (2013).
} 
performance measures. In particular, PSY (2015b) demonstrate consistency of the strategy in estimating the origination and termination dates of bubbles when either single or multiple bubbles appear in the sample. The data generating processes considered in those exercises are based on the model proposed in PWY where asset prices follow a random walk in normal market conditions, switch to a mildly explosive process under bubble expansion, and return to martingale dynamics when the bubble implodes. In that model bubbles collapse abruptly within one sample period, an assumption made largely for analytic convenience but lacking empirical realism. Casual inspection of the trajectories of financial asset bubbles typically reveal a more complex process of market correction and reversion. For instance, Figure 1 shows monthly Nasdaq price-dividend ratio from January 1991 to December 2005 with origination and termination dates as determined in PWY. The observed trajectory shows that the collapse process of the Nasdaq in the early 2000s is a complex one that is neither immediate nor monotonic. Implosion of the famous Dot Com bubble ${ }^{4}$ does not conclude within a single time period but involves many months of realignment.

Historical episodes of collapse have been classified in the literature into 'sudden', 'disturbing', or 'smooth' crisis events (Rosser, 2000; Huang et al., 2010). 'Sudden crises' characterize precipitate declines and correspond to the PWY model of abrupt declines in prices. In 'smooth crises' (a somewhat oxymoronic description used in the literature), prices fall smoothly with a moderate but persistent decline. 'Disturbing crises' are considered to be intermediate in form between these two extremes.

Figure 1: The monthly price-dividend ratio of the NASDAQ composite index for the sample period from January 1991 to December 2005, showing the bubble period detected by the PWY algorithm.

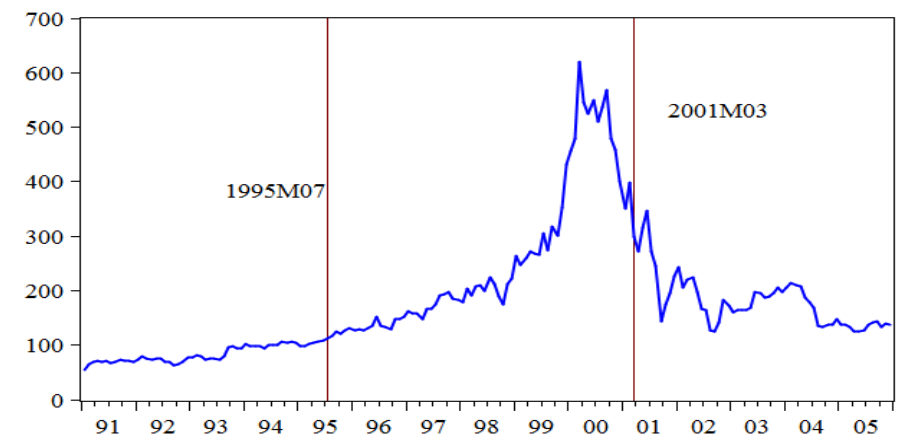

The data generating process used in PSY is limited to sudden crises and therefore lacks realism for other crisis scenarios, potentially affecting the asymptotic validity and finite sample performance of the testing algorithm under such conditions. The present paper addresses this concern by providing limit behavior and studying finite sample performance of the PSY strategy under more realistic bubble generating processes that allow for various forms of implosion that

\footnotetext{
${ }^{4}$ The presence of speculative bubble behavior in the Dot Com market around this sample period has been documented in PWY.
} 
fall into the above categories. The process considered here, given in (2) below, is an extension of the model proposed in PWY (2011) and used recently in Harvey, Leybourne, Sollis, and Taylor (2016; HLST). The model involves normal market and bubble exuberance dynamics similar to the PWY model while also allowing for drift during normalcy, whereas bubble implosion is modeled by a (stationary) mildly integrated process that is intended to capture elements of the mean reversion process that occur as prices collapse to normal market levels concordant with past and present fundamentals. This extension of the bubble collapse mechanism to allow for transitional dynamics was envisaged in the original formulation of the PWY model ${ }^{5}$ but was not pursued in that work. Figure 2 displays a typical realization of the PWY process and several realizations of the new process (2) with different collapse speeds and durations. As is evident in these graphs, the new bubble model is flexible and can produce richer dynamic trajectories in the collapse period. The PSY procedure is shown to be consistent and to have satisfactory finite sample performance in estimating the origination and termination dates of bubbles under the more realistic data generating process. This evidence provides additional support for the testing algorithm and reassurance to practioners of its suitability in bubble monitoring and detection under a variety of crisis scenarios.

Figure 2: Typical bubble collapse patterns generated by a mildly integrated process, giving sudden, disturbing, and smooth correction trajectories. Model parameters in (2) are set as $T=100, \eta=1, \alpha=0.7, d_{B T}=0.2, \beta=0.1, d_{C T}=\lfloor 0.01 T\rceil$ for sudden collapse, $\beta=0.5, d_{C T}=$ $\lfloor 0.10 T\rceil$ for disturbing collapse, and $\beta=0.9, d_{C T}=\lfloor 0.20 T\rceil$ for smooth collapse.

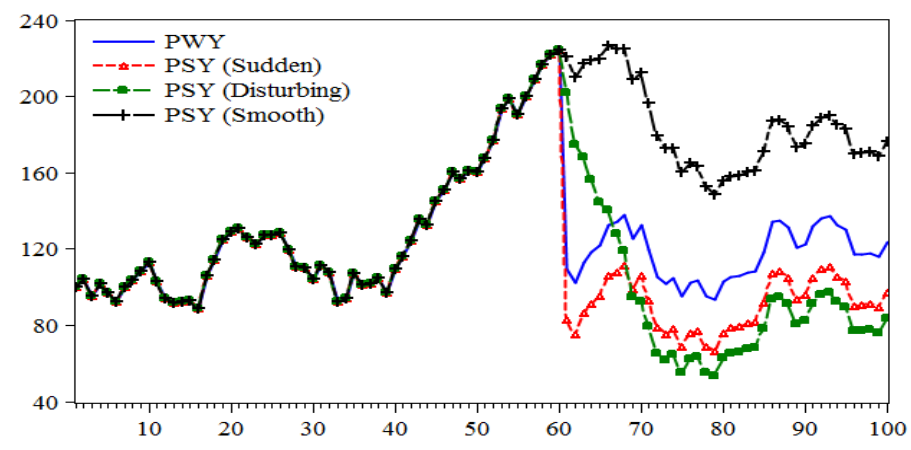

An important feature of the new model is the embodiment of a recovery date break point in the process. Market recovery is defined as the date asset prices return to their normal martingale path, effectively the switch point from the mildly integrated collapse process to the martingale path. A second aim of the paper is to address the econometric issues associated with consistent estimation of the recovery date. For the simplified PWY bubble process, market recovery coincides with bubble implosion because immediate market correction ensures that the asset price returns abruptly to its martingale path up to a term of $O_{p}(1)$. In the new model, the date of bubble implosion (the switch point from exuberant behavior to market correction behavior) differs from

\footnotetext{
${ }^{5}$ See the discussion following equation (14) of PWY.
} 
the market recovery date. By construction, the market recovery date of the new process is the conclusion point of the mildly integrated collapsing process (see Figure 3). This feature of the model leads to new practical issues such as the existence and nature of the collapsing regime and the econometric estimation of the crisis recovery date.

Figure 3: Turning points of different bubble generating processes showing abrupt correction (PWY) and mildly integrated correction with a separate market recovery date.

(a) PWY

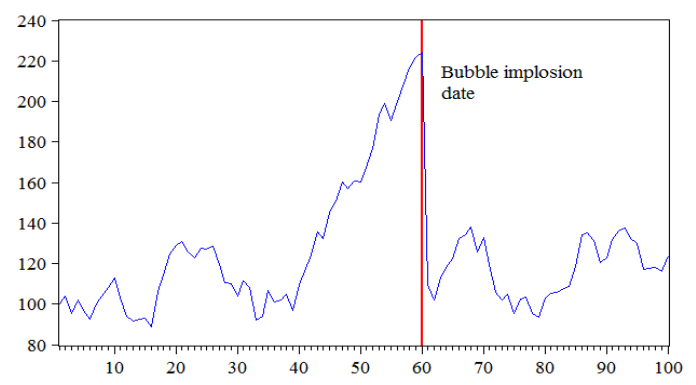

(b) PSY

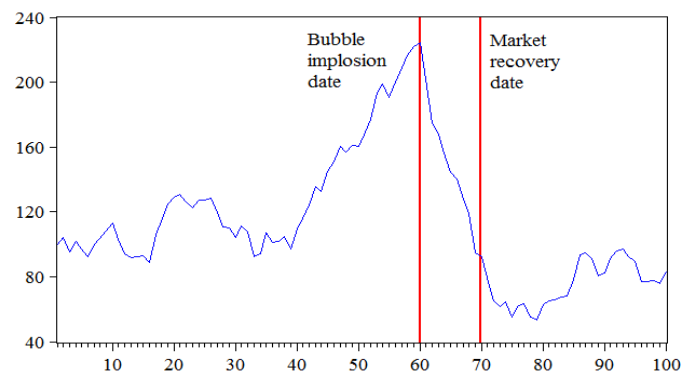

Econometric detection of the crisis regime is possible because the collapse process, modeled here by a mildly integrated process, is embedded in a long sample period that includes multiple regimes of martingale and explosive behavior. Doubly-recursive tests like those in PSY are well suited to deal with such break analysis. In an important related literature on testing for stationarity Leybourne, Kim and Taylor (2007) suggested doubly-recursive tests for unit roots against stationary alternatives. In their work on bubble break point testing HLS (2015) perform sequential procedures based on PWY tests, HB (2012) Chow tests, and a union of rejections strategy (combining PWY and HB tests) to identify bubble behavior. The HLS (2015) model uses a simple collapse process like that of PWY - either an abrupt collapse (like PWY) or an immediate transition to normal market behavior (a unit root process initialized at the last period of the explosive regime). In work that is much closer in spirit to that of the present paper and the extension suggested in PWY, HLST (2016) use a 4-regime model that incorporates an intermediate stationary regime to model a collapsing bubble.

A second contribution of the paper is the development of a new algorithm for detecting crises or bubble implosion and estimating their associated multiple turning points. Specifically, we recommend applying the recursive (or doubly-recursive) PSY test to data that is arranged in reverse order to the original series. This reverse regression strategy is suited to detecting either a single market crash or multiple crashes, included those most commonly occuring cases where the number of crashes is unknown. In contrast to PSY and PWY (2011) but similar to HLST (2016), the procedure is primarily designed for ex post analysis rather than real-time monitoring.

A challenging aspect with the approach proposed by HLST (2016) is feasibility. Their procedure requires that hypothesis tests be conducted between turning points. But in practical work, bubble expansions and contractions are often short-lived, producing insufficient observations for 
unit root tests to be conducted or to have good performance (particularly against stationary alternatives). This limitation of the HLST procedure is expected to be most apparent in cases of sudden and disturbing crises where changes can occur rapidly. The double recursive procedure used in the present work is not as affected by this limitation. The second major difference between the HLST and present procedure relates to prior knowledge concerning the number of bubble episodes. HSLT requires the number of bubble episodes to be known beforehand so that one can estimate the turning points before conducting sequential tests, whereas the method proposed in the present paper estimates the number of bubble/crisis episodes and turning points simultaneously. This is a useful advance for practical work as the number of bubble (crisis) episodes are usually unknown in advance. Assuming the wrong number of bubble episodes will result in miscalculation of the turning points and hence incorrect hypothesis testing results.

We demonstrate that the new procedure consistently estimates the origination and termination/recovery dates of crises. It has good finite sample performance in simulations and helps to reduce bias in some of the date estimates. A real-time version of the strategy is provided that is suited for practical implementation and ongoing policy analysis. In view of the technical complications arising from multiple collapse processes, from the presence of a unknown recovery date, and from the endogeneity introduced by reverse regression, the limit theory of the date stamping procedures involves substantial extension of earlier work in PWY (2011) and PSY (2015b).

Reverse recursive regression, like the forward regression approach of PSY, is a reduced form methodology. The collapse phase of a bubble is modeled as a transient mechanism in terms of a mildly integrated autoregression whose duration is data-determined. This mechanism accords with the mildly explosive process that captures the data-determined expansionary phase of the bubble. Both mechanisms may therefore be interpreted as mild deviations from martingale behavior and efficient market modeling. The reduced form specification facilitates estimation and testing. But it also enables some connections to be built with work that has been done in economics on modeling bubbles.

While there is no general theory or consensus on bubbles in economics or finance that explains their emergence and termination and that provides a structural model for estimation and testing, some recent work has explored behavioral, game theoretic, and learning mechanisms to capture certain elements of bubbles. Akerlof and Shiller (2009), for instance, advance a behavioralistic description of how psychological forces such as confidence, fear and greed can drive financial and real estate markets into regions where market prices are no longer supported by fundamentals. While Akerlof and Shiller provide no modeling apparatus, the divergences from efficient markets that they describe may be well represented by mildly explosive and mildly integrated departures from normal martingale behavior where all the driver elements are in balance with fundamentals. An alternative Bayesian perspective that also embodies investor attitudes has recently been advanced by $\mathrm{Li}$ and Xue (2009) to explain the 1990s Nasdaq bubble. They suggest "new economy thinking" as a primary driver of "rational investor" exuberance. Using a model of economic growth in which total factor productivity undergoes a structural break in the mid 1990s, these authors explain investor exuberance in terms of the "belief evolution" that occurs with Bayesian updating in response to the structural break. Such a structural break in productivity and the learning and uncertainty that it induces may be represented by a structural 
break in the reduced form that leads again to a mild departure from martingale behavior that can be modeled as in our approach. In other work, Abreu and Brunnemeier (2003) provide a dynamic gaming explanation for investors formulated under the assumption of a known terminal date to justify sustained departures from fundamentals. According to these authors, "rational investors" engage in gaming strategies that can lead to longlasting departures from fundamentals even though there is widespread recognition that prices are too high and must ultimately fall in line with fundamentals by the terminal date. This structural explanation of departures from normal market behavior can again be captured in terms of mild (reduced form) departures from the martingale behavior which links prices to fundamentals, thereby leading to both expansionary and contractionary phases of a bubble.

While none of the work just described is formulated in a way that is well suited to econometric implementation and structural model testing, all of these ideas may be captured in a reduced form framework where evolution in the coefficients embodies the various behavioralistic, learning, or gaming elements that underlie the suggested structural mechanisms. The absence of an agreed structural theory suited to econometric implementation is precisely the reason we work with reduced form models. Such models provide a natural mechanism for detecting change points that signify emergence and termination of bubble behavior. These models represent the (reduced form) outcome of many different structural models, and they have the powerful advantage, in view of their parsimony, of detecting and dating origination and termination.

We illustrate the use of the new strategy for crisis identification, along with the PSY strategy for bubble detection, in an application to the Nasdaq stock market over 1973M01-2013M08. While the PSY test suggests that the Dot Com bubble originated in December 1996, the reverse procedure finds that implosion occurred in February 2000 and the market recovered from December 2000, with a further correction in 2004M02-M04. Over this long historical series, the PSY procedure identifies two other bubble incidents (the 1983 bubble episode and the subsequent 1980s bubble leading up to the famous 'black Monday' crash of 1987). The reverse procedure also detects the 1973 stock market crash.

The rest of the paper is organized as follows. Section 2 introduces the PSY procedure for bubble monitoring and the reverse procedure of crisis detection. Section 3 derives the limit theory for the PSY strategy and the reverse procedure under the new bubble generating process that allows for flexibility in the collapse mechanism. Finite sample performance is studied in Section 4. An empirical application to long historical Nasdaq series is conducted in Section 5. Section 6 concludes. Two appendices contain supporting lemmas and derivations for the limit theory, which deals with both forward and reverse regression asymptotics. Complete details of the derivations and supporting lemmas are given in a technical supplement to the paper (Phillips and Shi, 2016) which is available online at Cambridge Journals Online (journals.cambridge.org/ect).

\section{Econometric Methods}

The following development uses models in which a single bubble occurs. Extension of the methods to cases where there are multiple bubbles may be established using PSY (2015a,b) and for brevity these are not provided here. 
We denote the bubble origination and collapse dates by $T_{e}$ and $T_{c}$, so that $B=\left[T_{e}, T_{c}\right]$ is the bubble period and $N_{0}=\left[1, T_{e}\right)$ and $N_{1}=\left(T_{c}, T\right]$ represent normal periods before and after the bubble episode. In this change point framework, the PWY bubble model has the form

$$
X_{t}=\left\{\begin{array}{ll}
X_{t-1}+\varepsilon_{t}, & t \in N_{0} \\
\delta_{T} X_{t-1}+\varepsilon_{t}, & t \in B \\
X_{T_{c}}^{*}+\sum_{i=T_{c}+1}^{t} \varepsilon_{i}, & t \in N_{1}
\end{array}, \varepsilon_{t} \sim i i d\left(0, \sigma^{2}\right)\right.
$$

where $t=1,2, \ldots, T, \delta_{T}=1+c_{1} T^{-\alpha}$ with $c_{1}>0$ and $\alpha \in[0,1), X_{T_{c}}^{*}=X_{T_{e}}+X^{*}$ with $X^{*}=O_{p}(1)$, and $X_{0}=o_{p}(1)$. Asset prices are assumed to be a pure random walk during normal periods. During market exuberance, asset prices follow a mildly explosive process. An abrupt collapse occurs at $T_{c}$, which brings the asset price back to the level when the bubble originated (i.e. $X_{T_{e}}$ ) plus a random perturbation $X^{*}$. The asset price then continues its martingale path towards the end of the sample period.

The new generating process considered here differs from the PWY model in three respects. First, it includes an asymptotically negligible drift in the martingale path during normal periods. Second, the collapse process is modeled directly as a transient mildly integrated process (Phillips and Magdalinos, 2007) that covers an explicit period of market collapse. Third, a market recovery date is introduced to capture the return to normal market behavior. The idea of using a transient process for the collapse period was suggested, but not pursued, in PWY (2011). The model has the following specification

$$
X_{t}=\left\{\begin{array}{ll}
c T^{-\eta}+X_{t-1}+\varepsilon_{t}, & t \in N_{0} \cup N_{1} \\
\delta_{T} X_{t-1}+\varepsilon_{t}, & t \in B \\
\gamma_{T} X_{t-1}+\varepsilon_{t}, & t \in C
\end{array},\right.
$$

where $B=\left[T_{e}, T_{c}\right]$ is the bubble episode as before, $C=\left(T_{c}, T_{r}\right]$ is the collapse period, $T_{r}$ is the date of market recovery, and $N_{0} \cup N_{1}=\left[1, T_{e}\right) \cup\left(T_{r}, T\right]$ are the normal market periods. Following PSY (2015a), the asset price process during $N_{0} \cup N_{1}$ involves an asymptotically negligible deterministic trend $\left(c T^{-\eta} t\right.$ with constant $c$ and some $\left.\eta>1 / 2\right)$ which adds a small drift to the normal martingale path. ${ }^{6}$ Both autoregressive coefficients $\delta_{T}=1+c_{1} T^{-\alpha}$ (with $c_{1}>0$ and $\alpha \in\left[0,1\right.$ )) and $\gamma_{T}=$ $1-c_{2} T^{-\beta}$ (with $c_{2}>0$ and $\beta \in[0,1)$ ) involve mild deviations from unity in the sense of Phillips and Magdalinos (2007), one $\left(\delta_{T}\right)$ in the explosive direction and the other $\left(\gamma_{T}\right)$ in the stationary direction. For given $c_{2}>0$, the speed of collapse is controlled by the parameter $\beta$. The smaller is $\beta$, the faster is the implosion rate during the collapse period $C$.

Similar to (2), the model in HLS (2012) contains four regimes. Instead of using local to unity specifications, HLS assume that the bubble and crisis regimes have fixed coefficients, modeling $X_{t}=\mu+u_{t}$ with

$$
u_{t}=\left\{\begin{array}{cc}
u_{t-1}+\varepsilon_{t} & t \in N_{0} \cup N_{1} \\
\left(1+\delta_{1}\right) u_{t-1}+\varepsilon_{t} & t \in B \\
\left(1-\delta_{2}\right) u_{t-1}+\varepsilon_{t} & t \in C
\end{array},\right.
$$

\footnotetext{
${ }^{6}$ See Phillips, Shi and Yu (2014) for detailed discussions of the specification and properties of the drift term. For ease of notation, we assume the drift term to be the same for $N_{0}$ and $N_{1}$. Provided that the condition $\eta>1 / 2$ is satisfied, allowing different drift values for $N_{0}$ and $N_{1}$ will not affect the analysis.
} 
so that regime $B$ is purely explosive with coefficient $1+\delta_{1}>1$ and the collapse regime $C$ is stationary with $1-\delta_{2}<1$. As in $(2)$, this formulation captures the idea suggested in PWY of a transient adjustment from regime $B$ back to normalcy, but the coefficients are not localized to unity and the model lacks a drift in the martingale regime.

It is noted that while the autoregressive coefficients in (2) depend on sample size, they do not in the HLS model. Empirical considerations often indicate that the explosive (collapse) rate is sample size or frequency dependent. As an example, we take the NASDAQ price index during the Dot Com bubble period (1995 -1999 for bubble expansion and 2000 for bubble collapse) at both monthly and daily frequency. ${ }^{7}$ For the bubble expansion phase, we have 60 observations for monthly and 1300 observations for daily data. The estimated autoregressive coefficients for the monthly and daily NASDAQ index are 1.042 and 1.001. For the bubble collapsing period, there are 12 and 260 observations, respectively, for the monthly and daily data. The estimated autoregressive coefficient for 2000 is 0.721 using monthly data and 0.993 with daily data. It is obvious that both the explosive and reverting elements (0.042 and 0.279 for monthly and 0.001 and 0.007 for daily) are inversely related to the sample size. The new DGP makes the link explicit by allowing the explosive and collapse specification rates to depend on $T$.

In (2) the localized specification $\gamma_{T}=1-c_{2} T^{-\beta}$ for the autoregressive coefficient during the collapse period allows for flexibility in possible collapse trajectories while retaining a vicinity of unity or near martingale flavor. In particular, the data generating process (2) is capable of generating abrupt collapses like the PWY process (1) if the value of $\beta$ is small and the collapse duration (i.e. $T_{r}-T_{c}$ ) is short. But the model can also generate smooth, slow or turbulent trajectories of correction for which the market collapse duration lasts longer, corresponding to quite different collapse processes in practice. Figure 2 shows a realization of the PWY process against some typical realizations of the mechanism (2) for various values of the parameters that indicate some of these possibilities.

\subsection{The PSY strategy for bubble origination and collapse dates}

The null hypothesis of the PSY (2015a) test is a unit root process with an asymptotically negligible drift, namely

$$
X_{t}=k T^{-\gamma}+X_{t-1}+\varepsilon_{t}, \text { with constant } k \text { and } \gamma>1 / 2 .
$$

The PSY strategy conducts a backward sup Dickey Fuller (BSDF) test for each observation of interest, which we briefly explain here for completeness. Let $f_{1}$ and $f_{2}$ be the (fractional) starting and ending points of the DF regression. The regression model includes an intercept but no time trend ${ }^{8}$ such that

$$
\Delta X_{t}=\mu+\rho X_{t-1}+\varepsilon_{t}, \varepsilon_{t} \stackrel{i . i . d}{\sim}\left(0, \sigma^{2}\right)
$$

\footnotetext{
${ }^{7}$ The Dot Com bubble is one of most famous bubble episodes in recent history and the rate of explosive behavior is usefully representative of historical bubble episodes. The selected sample period is based on the findings of Phillips, Wu and $\mathrm{Yu}$ (2011) and the current paper. We estimate an AR(1) model for monthly data and an AR(1)$\operatorname{GARCH}(1,1)$ for daily data.

${ }^{8}$ See Phillips, Shi and Yu (2014) for a detailed discussion of regression model specification for right-tailed unit root tests.
} 
where $t=\left\lfloor f_{1} T\right\rfloor, \ldots,\left\lfloor f_{2} T\right\rfloor$. The corresponding DF statistic sequence is $D F_{f_{1}}^{f_{2}}$.

Suppose interest focuses on the properties of the generating process at observation $t:=\lfloor f T\rfloor$ where $f$ is the sample fraction corresponding to $t$. We are particularly interested in whether there is a unit or explosive root in the process at this observation, therefore focusing attention on the upper tail (right side) of the distribution. The backward sup DF test (denoted BSDF) therefore calculates the sup of the DF statistics computed recursively over a sample sequence whose end point (expressed in fractional form) $f_{2}$ is fixed at $f$ and whose start point $f_{1}$ runs backwards from $f-f_{0}$ to 0 , where $f_{0}$ is the smallest window size in these regressions. Specifically, we define

$$
B S D F_{f}\left(f_{0}\right)=\sup _{f_{1} \in\left[0, f-f_{0}\right], f_{2}=f}\left\{D F_{f_{1}}^{f_{2}}\right\}, \text { with } f \in\left[f_{0}, 1\right],
$$

giving the statistic at $t$ (or sample fraction $f$ ) using a minimum window of size $f_{0}$.

To identify a mildly explosive bubble episode, the BSDF statistic is compared to its corresponding right-tail critical value. Let $s c v\left(\beta_{T}\right)$ be the $\left(1-\beta_{T}\right) 100 \%$ critical value of the $B S D F_{f}$ statistic and assume that $\operatorname{scv}\left(\beta_{T}\right) \rightarrow \infty$ as $\beta_{T} \rightarrow 0$. For practical implementation, $\beta_{T}$ is often fixed at $0.01,0.05$ or 0.1 . The origination (termination) date of bubble expansion is then calculated as the first chronological observation whose BSDF statistic exceeds (falls below) its corresponding critical value. The (fractional) dates of bubble emergence (origination) and collapse are denoted by $f_{e}$ and $f_{c}$ with corresponding estimates $\hat{f}_{e}$ and $\hat{f}_{c}$ which are defined in terms of first crossing times. Specifically,

$$
\begin{aligned}
& \hat{f}_{e}=\inf _{f \in\left[f_{0}, 1\right]}\left\{f: B S D F_{f}\left(f_{0}\right)>\operatorname{scv}\left(\beta_{T}\right)\right\} \\
& \hat{f}_{c}=\inf _{f \in\left[\hat{f}_{e}+L_{T}, 1\right]}\left\{f: B S D F_{f}\left(f_{0}\right)<\operatorname{scv}\left(\beta_{T}\right)\right\} .
\end{aligned}
$$

It is often useful to define a bubble as having a required minimum duration to eliminate potentially misleading information from short term blips in the data. PSY (2015a) used a minimum duration based on a slowly varying function such as $\log T$ to distinguish bubbles. In sample fraction form we can set $L_{T}=\delta \log (T) / T$ where $\delta$ is a sample-frequency dependent parameter (so that $\delta$ is greater for monthly data than quarterly or annual data). The sample fraction $L_{T}$ is used in defining the collapse date $\hat{f}_{c}$ in $(2)$ as the first crossing time following the minimum duration period of the bubble, $\hat{f}_{e}+L_{T}$, when the test sequence recursion $B S D F_{f}\left(f_{0}\right)$ falls below the critical value $\operatorname{scv}\left(\beta_{T}\right)$.

\subsection{Reverse recursion tests for crisis origination and termination}

For identifying crisis episodes, we suggest applying the BSDF test to data $X_{t}^{*}$ arranged in reverse order to the original series $X_{t}$, so that $X_{t}^{*}=X_{T+1-t}$, for $t=1,2, \ldots, T$. If asset prices follow model (2), the reversed series $X_{t}^{*}$ satisfies the following dynamics

$$
X_{t}^{*}=\left\{\begin{array}{ll}
-c T^{-\eta}+X_{t-1}^{*}+v_{t}, & t \in N_{0} \cup N_{1} \\
\delta_{T}^{-1} X_{t-1}^{*}+\delta_{T}^{-1} v_{t}, & t \in B \\
\gamma_{T}^{-1} X_{t-1}^{*}+\gamma_{T}^{-1} v_{t}, & t \in C
\end{array} .\right.
$$




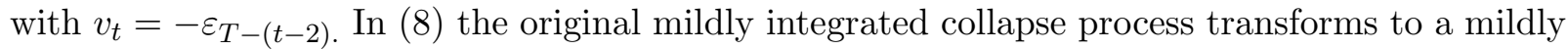
explosive process and vice versa. Hence, detecting crisis episodes in $X_{t}$ is equivalent to testing for mildly explosive behavior in $X_{t}^{*}$.

The BSDF statistic for crisis episode detection is defined as

$$
B S D F_{g}^{*}\left(g_{0}\right) \text { with } g \in\left[g_{0}, 1\right] \text { and } g=1-f \text {, }
$$

where $B S D F_{g}^{*}\left(g_{0}\right)$ is the BSDF statistic for observation (fraction) $g$ of $X_{t}^{*}$ where the recursion (in reverse direction) initiates with a minimum window size $g_{0}$. The market recovery date $\left(f_{r}\right)$ and crisis origination date $\left(f_{c}\right)$, both expressed in fractions of the original series sequence are then calculated as follows:

$$
\begin{aligned}
& \hat{f}_{r}=1-\hat{g}_{e}, \text { where } \hat{g}_{e}=\inf _{g \in\left[g_{0}, 1\right]}\left\{g: B S D F_{g}^{*}\left(g_{0}\right)>s c v^{*}\left(\beta_{T}\right)\right\} \\
& \hat{f}_{c}=1-\hat{g}_{c}, \text { where } \hat{g}_{c}=\inf _{g \in\left[\hat{g}_{e}, 1\right]}\left\{g: B S D F_{g}^{*}\left(g_{0}\right)<s c v^{*}\left(\beta_{T}\right)\right\},
\end{aligned}
$$

where $s c v^{*}\left(\beta_{T}\right)$ is the $\left(1-\beta_{T}\right) 100 \%$ critical value of the $B S D F_{g}^{*}\left(g_{0}\right)$ statistic, with $s c v^{*}\left(\beta_{T}\right) \rightarrow$ $\infty$ as $\beta_{T} \rightarrow 0$. According to these crossing times, market recovery $\left(\hat{f}_{r}\right)$ following a crash begins when normal market behavior changes to exuberance in the reverse series $\left(\hat{g}_{e}\right)$. Similarly, market collapse in the original series begins when exuberance in the reverse series shifts to collapse at $\left(\hat{g}_{c}\right)$. In many applications restrictions on crisis duration via the presence of a slowly varying function $L_{T}$ in these crossing time expressions will not be needed, especially when there is interest in the detection of abrupt crisis movements in the data.

Notice that the information set for calculating the $B S D F_{g}^{*}\left(g_{0}\right)$ statistic in the original series is $I_{f}^{R}=\left\{T_{f}, T_{f}+1, \ldots, T\right\}$ with $T-T_{f} \geq\left\lfloor T g_{0}\right\rfloor$, the minimum window size used in the reverse recursion. In the reverse time series $X_{t}^{*}$ this data corresponds to $I_{g}^{R}=\left\{1,2, \ldots, T_{g}=\lfloor T g\rfloor\right\}$ where $g=1-f$. Clearly, at any point in the sample $t<T$ the information set $\{t, t+1, \ldots, T\}$ contains future observations up to the sample end point $T$. Accordingly, we can regard this crisis detection strategy as an ex post identification tool. Nevertheless, there is a real time detector version of this algorithm that may be implemented in practical work as we now explain.

Specifically, the algorithm may be implemented on subsets of the data from any end point $K<T$. For example, suppose in the original series a bubble has been detected in the expansionary phase in real time so that $K>\lfloor f T\rfloor$. Then a question of major importance to all market participants and regulators is the timing of a market correction. To test for correction the above procedure may be implemented in reverse order from any sample point $t$ to assess evidence of a correction. In particular, suppose the current observation is $t=\lfloor\kappa T\rfloor=K$ for some $\kappa>0$. Reversing the series and writing $X_{s}^{*}=X_{K+1-s}$ for $s=1, \ldots,\lfloor g K\rfloor$ with $g \in[0,1]$, the recursive statistics $B S D F_{g}^{*}\left(g_{0}\right)$ may be calculated from $\left\{X_{s}^{*}\right\}_{s=1}^{\lfloor g K\rfloor}$ starting from some minimal window size $g_{0}$. In real time applications, $g_{0}$ will need to be small (in sample observation terms perhaps $\left\lfloor g_{0} T\right\rfloor \geq 6$ ) so that evidence for possible market correction is collected as early as possible. The main advantage of this approach (rather than testing for correction in the original series) is that right-sided unit root tests are typically much more sensitive to departures from the null than left-sided tests. In other words, the hypothesis of market correction is the existence of a mildly explosive process in the reverse series. 


\section{Asymptotics}

From PSY (2015b), the asymptotic distribution of the $B S D F_{f}\left(f_{0}\right)$ statistic under the null hypothesis (4) has the form

$$
F_{f}\left(W, f_{0}\right):=\sup _{f_{1} \in\left[0, f-f_{0}\right]}\left\{\frac{f_{w}\left[\int_{f_{1}}^{f} W(s) d s-\frac{1}{2} f_{w}\right]-\int_{f_{1}}^{f} W(s) d s \int_{f_{1}}^{f} d W}{f_{w}^{1 / 2}\left\{f_{w} \int_{f_{1}}^{f} W(s)^{2} d s-\left[\int_{f_{1}}^{f} W(s) d s\right]^{2}\right\}^{1 / 2}}\right\},
$$

where $W$ is a standard Wiener process. The reverse regression asymptotics are given in the following form.

Theorem 1 When the regression model includes an intercept and the null hypothesis is (4), the limit distribution of the $B S D F_{g}^{*}\left(g_{0}\right)$ statistic is:

$$
F_{g}\left(W, g_{0}\right):=\sup _{g_{1} \in\left[0, g-g_{0}\right]}\left\{\frac{g_{w}\left[\int_{1-g}^{1-g_{1}} W(s) d W+\frac{1}{2} g_{w}\right]+\int_{1-g}^{1-g_{1}} W(s) d s \int_{1-g}^{1-g_{1}} d W}{g_{w}^{1 / 2}\left\{g_{w} \int_{1-g}^{1-g_{1}} W(s)^{2} d s-\left[\int_{1-g}^{1-g_{1}} W(s) d s\right]^{2}\right\}^{1 / 2}}\right\} .
$$

See Appendix A and the Online Supplement for the proof. Notice that the $B S D F_{g}^{*}\left(g_{0}\right)$ statistic has a noncentral asymptotic distribution. The noncentrality arises from the endogeneity induced by the non-martingale implications of reverse regression, viz., the error component $\sum_{j=2}^{T} X_{j-1}^{*} v_{j}$ which, by virtue of the construction of the series $X_{t}^{*}$ and $v_{t}$, equals $-\sum_{j=2}^{T} X_{T-j+2} \varepsilon_{T-j+2}$ for which $\mathbb{E}\left(X_{T-j+2} \varepsilon_{T-j+2}\right) \neq 0$.

\subsection{The $B S D F_{f}\left(f_{0}\right)$ statistic}

The asymptotic properties of the BSDF statistic under the PWY bubble model are given in PSY (2015b). Here we derive the limit theory for the BSDF statistic under the more realistic bubble process (2) allowing for various forms of financial contraction captured by the parameterization within the collapse process. The derivations involve a non-trivial extension of the limit theory of PSY (2015b) to account for the additional regime, the drift in the normal martingale process, and the new bubble collapse process.

Theorem 2 (BSDF detector) Under the alternative hypothesis of mildly explosive behavior in model (2), the limit forms of the BSDF $\left(f_{0}\right)$ statistic are as follows:

$$
B S D F_{f}\left(f_{0}\right) \sim \begin{cases}F_{f}\left(W, f_{0}\right) \\ O_{p}\left(T^{1-\alpha / 2}\right) \rightarrow+\infty & \text { if } f \in N_{0} \\ O_{p}\left(T^{\omega(\alpha, \beta)}\right)= \begin{cases}O_{p}\left(T^{\alpha / 2}\right) \rightarrow-\infty & \text { if } \alpha>\beta \text { and } 1+\beta<2 \alpha \\ O_{p}\left(T^{(1-\alpha+\beta) / 2}\right) \rightarrow-\infty & \text { if } \alpha>\beta \text { and } 1+\beta>2 \alpha \\ O_{p}\left(T^{(1-\beta+\alpha) / 2}\right) \rightarrow-\infty & \text { if } \alpha<\beta \text { and } 1+\alpha>2 \beta \\ O_{p}\left(T^{\beta / 2}\right) \rightarrow+\infty & \text { if } \alpha<\beta \text { and } 1+\alpha<2 \beta\end{cases} \end{cases}
$$


Theorem 2 shows that the $B S D F$ statistic diverges to infinity at rate $O_{p}\left(T^{1-\alpha / 2}\right)$ when $f \in B$ and is $O_{p}\left(T^{\omega(\alpha, \beta)}\right)$ when $f \in C$ where the order $\omega(\alpha, \beta)$ depends on the values of the rate parameters $(\alpha, \beta)$. These results differ from those under the PWY model analyzed in PSY (2015c) where the BSDF statistic diverges to infinity at rate $O_{p}\left(T^{1-\alpha / 2}\right)$ when $f \in B$, as above, but diverges to negative infinity at rate $O_{p}\left(T^{(1-\alpha) / 2}\right)$ when $f \in C$ in contrast to the rate $O_{p}\left(T^{\omega(\alpha, \beta)}\right)$ above, which depends on the relative strengths $(\alpha, \beta)$ of the bubble and collapse processes. In particular, when the collapse regime follows a mildly integrated process (rather than an abrupt collapse), for $f \in C$ the limit form of the BSDF statistic may diverge to positive or negative infinity depending on the relative speeds of the bubble expansion and collapse, which are controlled by the rate parameters $\alpha$ and $\beta$. These parameters then play a major role in the conditions for consistent estimation of the bubble origination and termination dates, as shown in the following result.

Theorem 3 (BSDF detector) Suppose $\hat{f}_{e}$ and $\hat{f}_{c}$ are the date estimates obtained from the backward sup DF statistic crossing times (6). Under the alternative hypothesis of mildly explosive behavior in model (2), if the following conditions hold

$$
\left\{\begin{array}{ll}
\frac{T^{\alpha / 2}}{s c v^{\beta}}+\frac{s c v^{\beta} T}{T^{1-\alpha / 2}} \rightarrow 0 & \text { if } \alpha>\beta \text { and } 1+\beta<2 \alpha \\
\frac{T^{(1-\alpha+\beta) / 2}}{s c v^{\beta} T}+\frac{s v^{\beta} T}{T^{1-\alpha / 2}} \rightarrow 0 & \text { if } \alpha>\beta \text { and } 1+\beta>2 \alpha \\
\frac{T^{(1-\beta+\alpha) / 2}}{s c v^{\beta} T}+\frac{s c v^{\beta} T}{T^{1-\alpha / 2}} \rightarrow 0 & \text { if } \alpha<\beta \text { and } 1+\alpha>2 \beta \\
\frac{T^{\beta / 2}}{s c v^{\beta} T}+\frac{s c v^{\beta} T}{T^{1-\alpha / 2}} \rightarrow 0 & \text { if } \alpha<\beta \text { and } 1+\alpha<2 \beta
\end{array} .\right.
$$

we have $\hat{f}_{e} \stackrel{p}{\rightarrow} f_{e}$ and $\hat{f}_{c} \stackrel{p}{\rightarrow} f_{c}$ as $T \rightarrow \infty$.

Theorem (3) shows that consistent estimation of the dates of bubble origination and collapse requires certain conditions on the expansion rate of the test critical value $\operatorname{scv}\left(\beta_{T}\right)$. In particular, depending on the values of the rate parameters $(\alpha, \beta)$, the critical value $\operatorname{scv}\left(\beta_{T}\right)$ needs to lie respectively in the intervals $\left(T^{\alpha / 2}, T^{1-\alpha / 2}\right),\left(T^{(1-\alpha+\beta) / 2}, T^{1-\alpha / 2}\right),\left(T^{(1-\beta+\alpha) / 2}, T^{1-\alpha / 2}\right)$, and $\left(T^{\beta / 2}, T^{1-\alpha / 2}\right)$ according as $\{\alpha>\beta$ and $1+\beta<2 \alpha\},\{\alpha>\beta$ and $1+\beta>2 \alpha\},\{\alpha<\beta$ and $1+\alpha>2 \beta$,$\} and \{\alpha<\beta$ and $1+\alpha<2 \beta\}$.

These conditions are more restrictive than the simple condition that applies in the PWY bubble model, where the expansion rate of $\operatorname{scv}\left(\beta_{T}\right)$ is only required to be lower than $T^{1-\alpha / 2}$. Importantly, theorem (3) reveals that the conditions for consistent dating of a bubble collapse become increasingly restrictive as the rate of bubble collapse becomes slower with larger values of the collapse rate parameter $\beta$ (so that $\gamma_{T}$ is closer to unity). In this case, as might be expected, when the collapse is slow rather than rapid, large values of $\beta$ make it harder for the algorithm to distinguish the explosive bubble regime from the collapse regime.

\subsection{The $B S D F_{g}^{*}\left(g_{0}\right)$ statistic}

We first derive the limit properties of the $B S D F_{g}^{*}\left(g_{0}\right)$ under the data generating process (8). Note that the volatility of $X_{t}^{*}$ differs in regimes $B$ and $C$, as is clear from the reverse model 
specification (8), and there is a switch in the interpretation of the regimes since the autoregressive coefficients for the explosive and stationary regimes are now $\gamma_{T}^{-1} \sim 1+c_{2} T^{-\beta}$ and $\delta_{T}^{-1} \sim 1-c_{1} T^{-\alpha}$, respectively. The mildly explosive rate is now governed by the parameter $\beta$ and the collapse rate is controlled by $\alpha$.

Theorem 4 (The $B S D F_{g}^{*}\left(g_{0}\right)$ statistic) Under the alternative hypothesis of mildly explosive behavior in model (8), the limit behavior of the $B S D F_{g}^{*}\left(g_{0}\right)$ statistic is as follows:

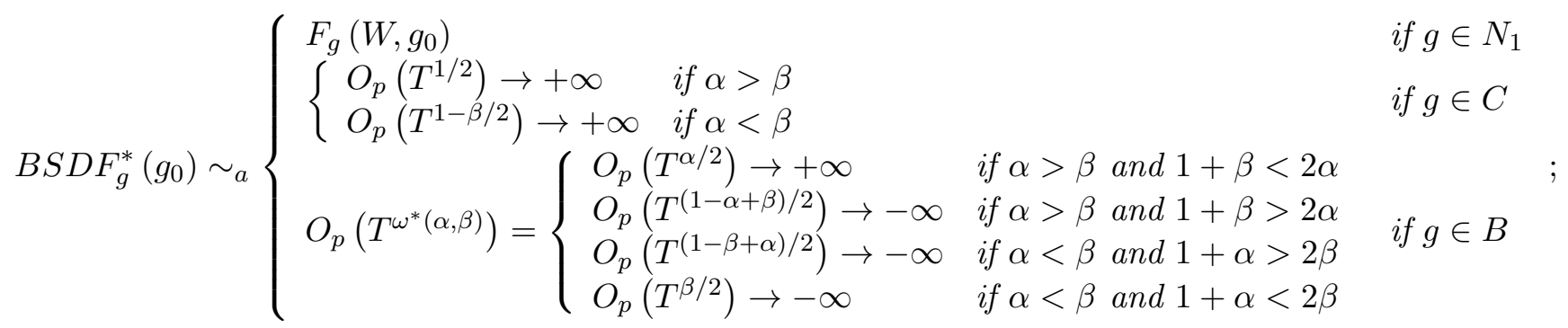

Like the BSDF statistic, the $B S D F_{g}^{*}\left(g_{0}\right)$ statistic diverges to positive infinity when $X^{*}$ is in the explosive regime (i.e. when $g \in C$ ). The rate of divergence is faster when $\alpha<\beta$ (i.e. $T^{1-\beta / 2}$ ) than it is when $\alpha>\beta$ (i.e. $\left.T^{1 / 2}\right)$. In this case, the divergence rate $O_{p}\left(T^{1-\beta / 2}\right)$ of the statistic also increases as $\beta$ decreases. Intuitively, as $\beta$ and $\alpha$ decrease both the collapse rate and bubble expansion rate increase, making detection of the collapse easier. In regime $B$, the limiting form of the $B S D F^{*}$ statistic has magnitude $O_{p}\left(T^{\omega^{*}(\alpha, \beta)}\right)$, which is the same as that for the BSDF statistic in regime $C$, so in this case the reverse and forward regressions are balanced.

Theorem 5 (The $B S D F_{g}^{*}$ detector) Suppose $\hat{f}_{c}$ and $\hat{f}_{r}$ are the date estimates obtained from the $B S D F_{g}^{*}\left(g_{0}\right)$ statistic crossing times rules (10). Under the alternative hypothesis of mildly explosive behavior in model (2), if

$$
\left\{\begin{array}{ll}
\frac{T^{\alpha / 2}}{s c v^{(}\left(\beta_{T}\right)}+\frac{s c v^{*}\left(\beta_{T}\right)}{T^{1 / 2}} \rightarrow 0 & \text { if } \alpha>\beta \text { and } 1+\beta<2 \alpha \\
\frac{T^{(1-\alpha+\beta) / 2}}{s c v^{*}\left(\beta_{T}\right)}+\frac{s v^{*}\left(\beta_{T}\right)}{T^{1 / 2}} \rightarrow 0 & \text { if } \alpha>\beta \text { and } 1+\beta>2 \alpha \\
\frac{T^{(1-\beta+\alpha) / 2}}{s c v^{*}\left(\beta_{T}\right)}+\frac{s c v^{*}\left(\beta_{T}\right)}{T^{1-\beta / 2}} \rightarrow 0 & \text { if } \alpha<\beta \text { and } 1+\alpha>2 \beta \\
\frac{T^{\beta / 2}}{s c v^{*}\left(\beta_{T}\right)}+\frac{s c v^{*}\left(\beta_{T}\right)}{T^{1-\beta / 2}} \rightarrow 0 & \text { if } \alpha<\beta \text { and } 1+\alpha<2 \beta
\end{array} .\right.
$$

we have $\hat{f}_{r} \stackrel{p}{\rightarrow} f_{r}$ and $\hat{f}_{c} \stackrel{p}{\rightarrow} f_{c}$ as $T \rightarrow \infty$.

To obtain consistent estimators of the crisis origination and termination dates, the expansion rate of $s c v^{*}\left(\beta_{T}\right)$ is required to fall respectively in the intervals $\left(T^{\alpha / 2}, T^{1 / 2}\right),\left(T^{(1-\alpha+\beta) / 2}, T^{1 / 2}\right)$, $\left(T^{(1-\beta+\alpha) / 2}, T^{1-\beta / 2}\right)$, and $\left(T^{\beta / 2}, T^{1-\beta / 2}\right)$ for the cases $\{\alpha>\beta$ and $1+\beta<2 \alpha\},\{\alpha>\beta$ and $1+\beta>2 \alpha\}$, $\{\alpha<\beta$ and $1+\alpha>2 \beta\}$, and $\{\alpha<\beta$ and $1+\alpha<2 \beta\}$. Despite their apparent complexity, these conditions generally accord with intuition because they tend to be less restrictive in the following cases: (i) as the value of $\alpha$ decreases (that is, as the collapse rate for $X_{t}^{*}$ becomes faster, or the mildly explosive rate for $X_{t}$ increases); and (ii) as the value of $\beta$ decreases (that is, as the explosive rate for $X_{t}^{*}$ increases, or the collapse rate of $X_{t}$ increases). 


\section{Simulation Evidence}

Extensive simulation studies were conducted in PSY (2015a,b) showing that the PSY dating strategy performs well under the PWY data generating process compared with other strategies. These simulations are extended here to explore finite sample performance under the new bubble generating process (2) that allows for multiple forms of collapse regime. We consider bubble detection performance as well as bubble origination and collapse date determination. These findings complement those of PSY for abrupt collapse conditions. We also investigate performance characteristics of the $B S D F^{*}$ test for crisis detection under the new bubble generating process.

The base parameter settings used here are the same as those in PSY (2015b), namely $X_{0}=$ 100, $\sigma=6.79$, and $c=c_{1}=c_{2}=1$, where $X_{0}$ and $\sigma$ are selected to match the initial value and sample standard deviation of the normalized S\&P 500 price-dividend ratio examined in PSY. Bubbles were identified using respective finite sample $95 \%$ quantiles obtained from simulations with 2,000 replications. The minimum window size has 19 observations and lag order is set to zero. The minimum window size is set according to the rule $f_{0}=0.01+1.8 / \sqrt{(T)}$ suggested in PSY (2015a). The simulations explore the effects of various settings for the mildly explosive and collapse regime rate parameters $(\alpha, \beta)$ that enter the new process $(2)$.

\subsection{The BSDF test for bubble origination and collapse dates}

As in PSY (2015b) we examine the proportion of successful bubble detection, along with the empirical mean and standard deviation (given in parentheses in the following tables) of the estimated origination and collapse dates. Successful detection of a bubble is defined as an outcome where the estimated origination date is greater than or equal to the true origination date and smaller than the true collapse date of that particular bubble (i.e. $f_{i e} \leq \hat{f}_{i e}<f_{i c}$ ). So the narrower is the interval $\left(f_{i e}, f_{i c}\right)$ the more challenging is the requirement. The duration of bubble expansion is restricted to have a minimum of 3 observations and to confirm the collapse of a bubble we require the test statistic $B S D F$ to go below its critical value for at least two consecutive periods. ${ }^{9}$

Table 1: Bubble detection rate and estimation of the origination and collapse dates (for different drift values). Parameters are set to: $X_{0}=100, \sigma=6.79, c=c_{1}=c_{2}=1, \alpha=0.60, \beta=$ $0.1, d_{B T}=\lfloor 0.20 T\rfloor, d_{C T}=\lfloor 0.01 T\rfloor, f_{e}=0.4, T=100$. Figures in parentheses are standard deviations.

\begin{tabular}{lllll}
\hline & DGP (PWY) & \multicolumn{2}{l}{ DGP (PSY) } & \\
\cline { 3 - 4 } & & $\eta=0.6$ & $\eta=1$ & $\eta=2$ \\
Bubble Detection Rate & 0.86 & 0.86 & 0.85 & 0.87 \\
$\hat{f}_{e}-f_{e}$ & $0.06(0.04)$ & $0.06(0.04)$ & $0.06(0.04)$ & $0.06(0.04)$ \\
$\hat{f}_{c}-f_{c}$ & $0.01(0.01)$ & $0.01(0.01)$ & $0.01(0.01)$ & $0.01(0.01)$ \\
\hline
\end{tabular}

Note: Calculations are based on 2,000 replications. The minimum window has 19 observations.

\footnotetext{
${ }^{9}$ Specifically, $\hat{f}_{e}=t$ if $B S D F_{t-2}<s c v, B S D F_{t-1}<s c v, B S D F_{t}>s c v, B S D F_{t+1}>s c v$, and $B S D F_{t+2}>c v$ and $\hat{f}_{c}=t$ if $B S D F_{t-3}>s c v, B S D F_{t-2}>s c v, B S D F_{t-1}>s c v, B S D F_{t}<s c v$ and $B S D F_{t+1}<s c v$.
} 
The first step is to investigate the impact of drift in the unit root process on bubble detection accuracy. The PSY estimate of the bubble origination date depends solely on past information. So when there is a single bubble in the sample period, the mildly integrated collapse regime, which occurs after bubble origination and expansion, should have no impact on the accuracy of the bubble origination estimate. But the presence of drift, even asymptotically negligible drift, in the unit root process that characterizes normal periods may well impact bubble origination detection.

In the simulation, we fix $\beta$ at 0.1 and the duration of bubble collapse $d_{C T}$ at $\lfloor 0.01 T\rceil$. This setting delivers an instantaneous one-period collapse when the sample size $T=100$, which resembles the abrupt collapse pattern of PWY. We let $\eta$ take the values $\{0.6,1,2\}$, which give corresponding drift values of $\{0.063,0.010,0.000\}$. For each parameter constellation, 2,000 replications were employed. As evident in Table 1, the parameter $\eta$ has no material impact on bubble detection when $\eta>0.5$ as here, which is concordant with asymptotic theory. Thus, the PSY strategy continues to deliver consistent estimates despite the inclusion of this type of drift. However, under these parameter settings, there is a six-observation delay in detecting the bubble, indicating some upward bias in the date estimates. On the other hand, just as in PSY, due to the sudden one-period collapse, the termination date of the bubble expansion is estimated with great accuracy.

The next step is to explore the influence of a mildly integrated collapse regime on the performance of the PSY strategy. The model specification of this regime allows for processes with different collapse speeds and durations. We consider three collapse patterns here: sudden collapse (i.e $\beta=0.1$ and $d_{C T}=\lfloor 0.01 T\rceil$ ), disturbing collapse (i.e. $\beta=0.5$ and $d_{C T}=\lfloor 0.10 T\rceil$ ), and smooth collapse (i.e. $\beta=0.9$ and $d_{C T}=\lfloor 0.20 T\rceil$ ). We let $\eta=1, \alpha=0.6, d_{B T}=0.2$. Figure 2 plots one typical realization of these three collapse processes.

Table 2 displays the estimation results of the PSY strategy under different data generating processes. We observe an increasing delay in bubble collapse date estimates (viz., $f_{c}$ ) as the collapse process becomes smoother. For instance, the bias $\left(\hat{f}_{c}-f_{c}\right)$ is three observations in a disturbing collapse regime but increases to eleven observations for a smooth collapse. The bubble origination date is unaffected by the collapse regime dynamics, as expected and indicated above.

Table 2: Bubble detection rate and estimation of the origination and collapse dates (under different collapse patterns). Parameters are set to: $X_{0}=100, \sigma=6.79, c=c_{1}=c_{2}=$ $1, \alpha=0.60, d_{B T}=\lfloor 0.20 T\rfloor, f_{e}=0.4, T=100, \beta=0.1, d_{C T}=\lfloor 0.01 T\rceil$ for sudden collapse, $\beta=0.5, d_{C T}=\lfloor 0.10 T\rceil$ for disturbing collapse, and $\beta=0.9, d_{C T}=\lfloor 0.20 T\rceil$ for smooth collapse. Figures in parentheses are standard deviations.

\begin{tabular}{lllll}
\hline & PWY & Sudden & Disturbing & Smooth \\
Bubble Detection Rate & 0.86 & 0.85 & 0.86 & 0.85 \\
$\hat{f}_{e}-f_{e}$ & $0.06(0.04)$ & $0.06(0.04)$ & $0.06(0.04)$ & $0.06(0.04)$ \\
$\hat{f}_{c}-f_{c}$ & $0.01(0.01)$ & $0.01(0.01)$ & $0.03(0.01)$ & $0.11(0.04)$ \\
\hline
\end{tabular}

Note: Calculations are based on 2,000 replications. The minimum window has 19 observations. 


\subsection{The $B S D F^{*}$ test for crisis origination and market recovery dates}

In the case of a bubble-led crisis such as model (2), the origination date of the crisis coincides with the date of collapse. Successful detection of a bubble-led crisis may then be defined as an outcome where the estimated collapse date is greater than or equal to the true bubble origination date and smaller than the true recovery date of that particular crisis (i.e. $f_{i e} \leq \hat{f}_{i c}<f_{i r}$ ). As earlier, we report the detection rate of a crisis, along with the empirical mean and standard deviation (shown in parentheses) of the estimated crisis origination and termination dates. For the crisis identification, we do not impose restrictions on the crisis duration; but, as in bubble identification, we require the test statistic $B S D F^{*}$ to go below its critical value for at least two consecutive observations to confirm a recovery. ${ }^{10}$

Table 3: Crisis detection rate and estimation of the collapse and recovery dates (with various expansion and collapse patterns). Parameters are set to: $X_{0}=100, \sigma=6.79, c=c_{1}=c_{2}=$ $1, d_{B T}=\lfloor 0.20 T\rfloor, f_{e}=0.4, T=100, \beta=0.1, d_{C T}=\lfloor 0.01 T\rceil$ for sudden collapse, $\beta=0.5, d_{C T}=$ $\lfloor 0.10 T\rceil$ for disturbing collapse, and $\beta=0.9, d_{C T}=\lfloor 0.20 T\rceil$ for smooth collapse. Figures in parentheses are standard deviations.

\begin{tabular}{llll}
\hline & Sudden & Disturbing & Smooth \\
$\alpha=0.6$ & & 0.97 & 0.89 \\
Crisis Detection Rate & 0.44 & $-0.03(0.01)$ & $0.00(0.05)$ \\
$\hat{f}_{c}-f_{c}$ & $-0.00(0.02)$ & $-0.03(0.02)$ & $-0.10(0.07)$ \\
$\hat{f}_{r}-f_{r}$ & $0.00(0.02)$ & & \\
\hline$\alpha=0.7$ & & 0.95 & 0.80 \\
Crisis Detection Rate & 0.41 & $-0.03(0.02)$ & $-0.01(0.08)$ \\
$\hat{f}_{c}-f_{c}$ & $-0.00(0.01)$ & $-0.04(0.03)$ & $-0.14(0.09)$ \\
$\hat{f}_{r}-f_{r}$ & $-0.00(0.00)$ & & 0.69 \\
\hline$\alpha=0.8$ & & 0.92 & $-0.02(0.09)$ \\
Crisis Detection Rate & 0.40 & $-0.04(0.03)$ & $-0.16(0.10)$ \\
$\hat{f}_{c}-f_{c}$ & $-0.02(0.05)$ & $-0.05(0.04)$ & \\
$\hat{f}_{r}-f_{r}$ & $-0.01(0.05)$ & & \\
\hline
\end{tabular}

Note: Calculations are based on 2,000 replications. The minimum window has 19 observations.

Table 3 reports the performance characteristics of the $B S D F^{*}$ test under sudden, disturbing, and smooth collapse processes with different bubble expansion rates. As is evident in the Table, the crisis detection rate is highest for disturbing collapses (97\% when $\alpha=0.6$ ) and lowest for sudden collapses $(44 \%$ when $\alpha=0.6$ ). These findings are explained by the fact that sudden crises have short duration and collapse date estimates come late, whereas in a disturbing crisis there is a delay before recovery which aids crisis detection. The crisis detection rate increases with the

\footnotetext{
${ }^{10}$ Specifically, $\hat{f}_{c}=t$ if $B S D F_{t-2}^{*}<r s c v, B S D F_{t-1}^{*}<r s c v, B S D F_{t}^{*}>r s c v$ and $\hat{f}_{r}=t$ if $B S D F_{t}^{*}>r s c v$, $B S D F_{t+1}^{*}<r s c v$, and $B S D F_{t+2}^{*}<r s c v$.
} 
rate of bubble expansion. As a case in point, when $\alpha$ decreases from 0.8 to 0.6 , the detection rate for disturbing collapses rises from $92 \%$ to $97 \%$.

In addition, we see that the estimated crisis origination date is generally biased downward. Moreover, the bias (i.e. $\hat{f}_{c}-f_{c}$ ) is larger for disturbing collapses than sudden and smooth collapses. Interestingly, the estimation accuracy of the crisis origination date also depends on the bubble expansion rate. Namely, the bias is marginally larger when the bubble expansion rate is slower. This is consistent with our earlier finding for the BSDF test where the estimated crisis origination (or bubble collapse) date is affected by the collapse pattern.

Estimates of the recovery date are evidently highly accurate for sudden collapses, whereas there is substantial downward bias for smooth collapses. For instance, when $\alpha=0.6$, there is a ten-period bias (earlier than the true date) in the estimates of $f_{r}$ under a smooth collapse, which accounts for $50 \%$ of the collapse duration. The bias for disturbing collapses is much smaller and is near zero bias for sudden collapses. For the smooth collapse, the bias increases as the bubble expansion rate becomes slower (with larger $\alpha$ ).

For further investigation, we extend the parameter specification in the case of disturbing collapses by varying $\beta$ from 0.3 to 0.7 and $d_{C T}$ from $5 \%$ of the total sample to $15 \%$. Consistent with expectations, the $B S D F^{*}$ strategy provides higher crisis detection rates when bubbles collapse faster and the crisis termination date is more accurately estimated when there is shorter collapse duration. Detailed results are provided in the online supplement (Phillips and Shi, 2016).

\subsection{Real Time Monitoring of Market Correction}

The goal of this use of reverse regression is to aid the ongoing detection of market recovery dates. We propose to implement the reverse procedure repeatedly for each observation starting from the date of the bubble collapse, that is $T_{c}$ in chronological time. The dating rules in (9) and (10) can always be rewritten as

$$
\begin{aligned}
& \hat{f}_{c}=\inf _{f \in\left[0,1-g_{0}\right]}\left\{f: B S D F_{f}^{* *}\left(g_{0}\right)>s c v^{* *}\left(\beta_{T}\right)\right\} \\
& \hat{f}_{r}=\inf _{f \in\left[\hat{f}_{c}, 1-g_{0}\right]}\left\{f: B S D F_{f}^{* *}\left(g_{0}\right)>s c v^{* *}\left(\beta_{T}\right)\right\}
\end{aligned}
$$

where $B S D F_{f}^{* *}\left(g_{0}\right)$ and $s c v^{* *}\left(\beta_{T}\right)$ are the reverse series of $B S D F_{g}^{*}\left(g_{0}\right)$ and $s c v^{*}\left(\beta_{T}\right)$. Suppose we conduct the reverse PSY test on a sample period running from some initial (reverse) observation 1 through to $K$ (which includes observation $T_{c}$ in chronological time) and identify one occurrence of market correction, namely $B S D F_{t^{\prime}-1}^{* *}>s c v^{* *}$ with $B S D F_{t^{\prime}}^{* *}<s c v^{* *}$, $B S D F_{t^{\prime}+1}^{* *}<s c v^{* *}$, and $t^{\prime} \geq T_{c}$ for some given critical value $s c v^{* *}$ in the reverse regression. Suppose, in addition, that no market correction is detected in samples from 1 to $s$, with $s<K$. Then, we conclude that $t^{\prime}$ is the date of market recovery (i.e. $\hat{f}_{r}=t^{\prime} / T$ ) and $K$ is the date at which the correction is detected in the data.

Table 4 reports the average delay of the estimated market recovery date $\left(\hat{f}_{r}-f_{r}\right)$ and the average delay in detecting this correction $\left(D D=K / T-T f_{0}\right)$. By construction, the delay in detecting the market correction is bounded below by the observations required to initiate the 
regression $\left\lfloor T f_{0}\right\rfloor-1$. For early detection, one would need to consider a small minimum window size. On the other hand, choosing too small a value for the minimum window size may result in inaccurate estimation of model parameters and lead to corresponding distortions in the market recovery date estimates. Hence, the choice of $f_{0}$ is important for performance of the monitoring procedure. In simulations we allowed the minimum window size $f_{0}$ to vary from 0.06 to 0.18 to assess sensitivities to the choice of $f_{0}$. The sample sizes considered are 100 and 200. The number of replications was 2,000 .

Table 4: The estimated dates of market recovery and the delays in detecting market correction. Parameters are set to: $X_{0}=100, \sigma=6.79, c=c_{1}=c_{2}=1, \alpha=0.6, d_{B T}=\lfloor 0.20 T\rfloor, f_{e}=$ $0.4, T=100$. Figures in parentheses are standard deviations.

\begin{tabular}{lllll}
\hline (1) Disturbing Crises & & & \\
& $f_{0}=0.06$ & $f_{0}=0.08$ & $f_{0}=0.12$ & $f_{0}=0.18$ \\
$T=100$ & & & \\
Crisis Detection Rate & 0.99 & 0.99 & 0.98 & 1.00 \\
$\hat{f}_{r}-f_{r}$ & $-0.08(0.03)$ & $-0.09(0.01)$ & $-0.09(0.01)$ & $-0.10(0.00)$ \\
$\mathrm{DD}$ & $0.05(0.01)$ & $0.07(0.00)$ & $0.11(0.00)$ & $0.17(0.00)$ \\
$T=200$ & & & \\
Crisis Detection Rate & 1.00 & 1.00 & 0.99 & 0.99 \\
$\hat{f}_{r}-f_{r}$ & $-0.09(0.02)$ & $-0.09(0.02)$ & $-0.10(0.01)$ & $-0.10(0.01)$ \\
DD & $0.06(0.00)$ & $0.08(0.00)$ & $0.12(0.00)$ & $0.18(0.00)$ \\
\hline $\begin{array}{l}\text { (2) Smooth Crises } \\
\text { ( }\end{array}$ & $f_{0}=0.06$ & $f_{0}=0.08$ & $f_{0}=0.12$ & $f_{0}=0.18$ \\
$T=100$ & & & \\
Crisis Detection Rate & 0.93 & 0.92 & 0.90 & 0.90 \\
$\hat{f}_{r}-f_{r}$ & $-0.13(0.07)$ & $-0.13(0.06)$ & $-0.14(0.06)$ & $-0.17(0.31)$ \\
DD & $0.06(0.03)$ & $0.08(0.03)$ & $0.12(0.03)$ & $0.18(0.02)$ \\
$T=200$ & & & & \\
Crisis Detection Rate & 0.98 & 0.96 & 0.96 & 0.94 \\
$\hat{f}_{r}-f_{r}$ & $-0.15(0.05)$ & $-0.16(0.05)$ & $-0.16(0.05)$ & $-0.18(0.03)$ \\
DD & $0.06(0.02)$ & $0.08(0.01)$ & $0.12(0.02)$ & $0.18(0.01)$ \\
\hline
\end{tabular}

Note: Calculations are based on 2,000 replications.

From Table 4, it is apparent that the procedure provides less accurate estimates for the market recovery date of a smooth crisis than for a disturbing crisis. For example, when sample size is 100, with a minimum window size of 12 observations, the estimated market recovery date of the disturbing crisis is five observations closer to the true recovery date than that of the smooth crisis - the bias of the estimates is -0.09 and -0.14 , respectively, for a disturbing and a smooth crisis. For both types of crisis, estimation accuracy of the market recovery date improves slightly with a smaller setting for $f_{0}$. For the disturbing crisis, the bias reduces from 10 observations to 8 observations when $f_{0}$ declines from 0.18 to 0.06 . 
While the successful detection rate of a disturbing crisis is not affected by the setting of the minimum window size, the SDR of a smooth crisis decreases slightly when the minimum window size increases. When the sample size increases from 100 to 200, although there is a slight increase in the successful detection rate, the estimated market recovery date is less accurate. For example, for a smooth crisis, when $f_{0}=0.12$, both the successful detection rate and the bias of the $f_{r}$ estimate increase (from $90 \%$ to $96 \%$ and from -0.14 to -0.16 ).

As expected, the average delay in detecting the correction is bounded below by $\left\lfloor T f_{0}\right\rfloor-1$. Table 4 shows that for a disturbing crisis the average value of DD is $f_{0}-0.01$ when $T=100$ and $f_{0}$ when $T=200$. For a smooth crisis, the value of DD is $f_{0}$ for both sample sizes. Furthermore, there is not much variation in the estimated DDs. This is reflected in close-to-zero standard deviations in the estimated DDs for both disturbing and smooth crises, although the standard deviations for the smooth crisis are slightly above those of the disturbing crisis.

In summary, the real-time monitoring strategy for market recovery seems to perform better with a smaller minimum window size. But such a practice does need to be implemented with care because too small a value of $f_{0}$ can potentially lead to size distortion. ${ }^{11}$

\section{Empirical Applications}

According to the monthly report of the World Federation of Exchanges ${ }^{12}$, Nasdaq is the second largest exchange in the world by market capitalization and trading volume. The Nasdaq composite stock index is a market capitalization weighted stock index of the common stocks and similar securities listed on the Nasdaq stock market. It is weighted heavily towards information technology companies and has been widely studied for speculative bubble behavior especially for periods at the turn of the century (among others, Phillips, Wu and Yu, 2011, Li and Xue, 2009, Shi and Song, 2016).

For the analysis here, we downloaded the Nasdaq composite index and NASDAQ dividend yield at the monthly frequency from Datastream International, starting from January 1973 to August 2013. The dividend index in the current period $(t)$, which is included as a proxy for stock market fundamentals ${ }^{13}$ is calculated by multiplying the price index by the dividend yield at period $t+1$.

We date the bubble and crisis episodes of the Nasdaq stock market using the Nasdaq pricedividend ratio. Results are reported in Figure 4. The left (right) panel is for bubble (crisis) identification plotting the BSADF statistic (BSADF*) sequence against its $95 \%$ corresponding critical value sequence. The finite sample critical value sequences are obtained by Monte Carlo

\footnotetext{
${ }^{11}$ The importance of the immediate past of a series on the test outcome depends on the difference between the last two consecutive observations. The larger the difference the greater the impact of the immediately preceding observation on the reject probability at the current observation. This difference is governed by the values of $\alpha$ and $\beta$ in the data generating process (2) and hence the impact can be viewed from both the limit theory and in the finite sample simulations.

${ }^{12}$ http://www. world-exchanges .org/statistics/monthly-reports

${ }^{13}$ All bubble detection procedures (including PWY, HB, PSY and HSLT) typically require controlling for market fundamentals. The importance of the market fundamental is sample and trajectory dependent and test rejection can be expected whenever there are sustained departures from fundamentals.
} 
Figure 4: The identified bubble and crisis episodes using the PSY (left) and the reverse (right) procedures. The lag order is one and minimum window size is 44 observations. The finite sample critical value sequences are obtained by Monte carlo simulation with 2,000 replications.

(a) Bubbles

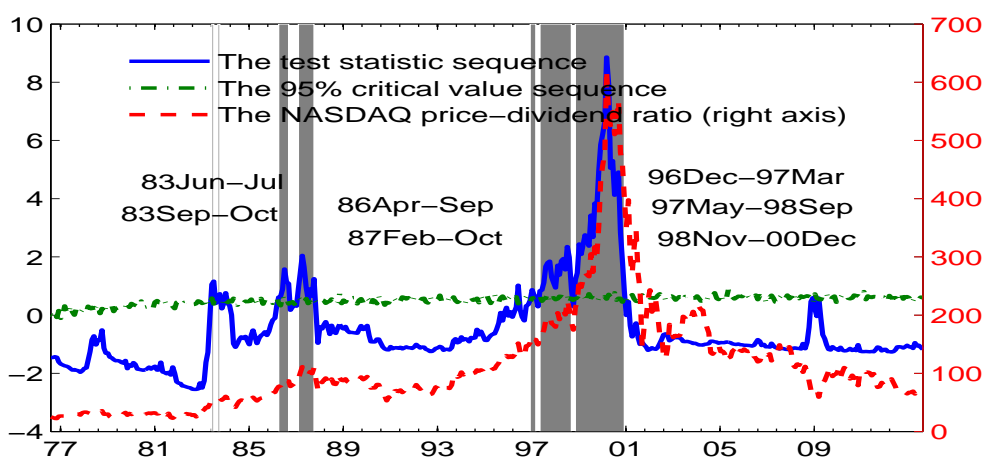

(b) Crises

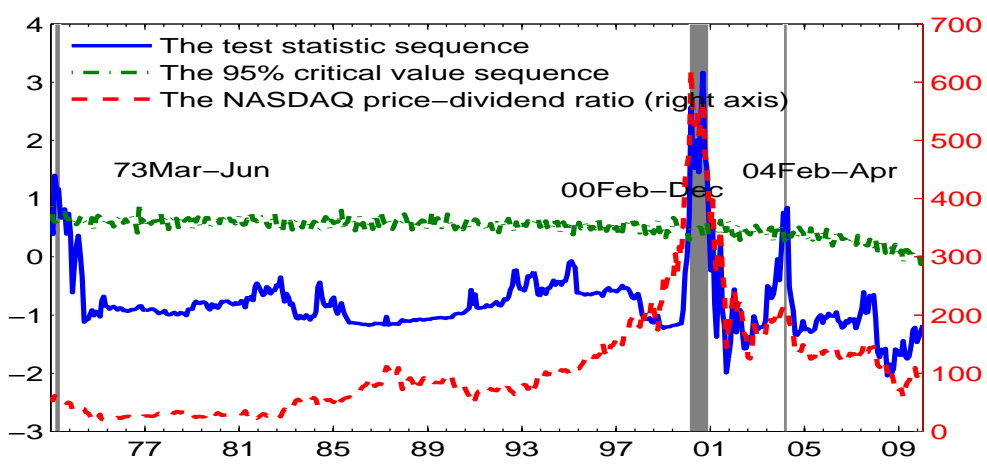

simulation with 2,000 replications. We set the smallest window size according to the rule of $0.01+1.8 / \sqrt{T}$ recommended by PSY $(2015 \mathrm{a})$, giving 44 observations and consider a lag order of one for the ADF regression.

According to Figure 4a, there is speculative bubble behavior in the stock market in 1986-1987 and 1996-2000, which led successively to the Black Monday crash in October 1987 and the Dot Com bubble crash in early 2000. The identified bubble origination and collapsing dates for the 1986-1987 episode are 1986M04-M09 and 1987M02-M10. The identified bubble period for the Dot Com episode using PSY is 1996M12-2000M12 (with some small breaks in between). Recall that the PSY procedure manifests some delay in detecting the bubble collapse date, especially in the case of a smooth collapse. Therefore, it is likely that the estimated collapse date exceeds the actual termination date of bubble expansion and the identified bubble expansion period covers partially the bubble collapse phase. Here, while the Nasdaq market collapses in March 2000, 
the estimated collapsing date is December 2000. There is a nine-observation delay in estimation. There is another short duration bubble episode in 1983 (1983M06-M07 and 1983M9-M10).

The reverse procedure results for crisis period detection are presented in Figure $4 \mathrm{~b}$. There are two major stock market downturns in the sample period. One is in 1973 and the other in early 2000s corresponding to the Dot Com bubble collapse. The 1973 episode lasts for four months, starting from 1973M03. The estimated collapse date of the Dot Com bubble episode obtained from the reverse procedure is 2000M02, which is closer to the peak of the Dot Com bubble (i.e. 2000M3) than the estimated collapsing date from the PSY procedure. Interestingly, therefore, the BSADF* test anticipates the crash but does so using the ex post subsequent crash data, of course.

The BSADF* procedure does not detect collapses for the 1983 and 1986-87 bubble episodes, which is perhaps unsurprising because simulations show that the crisis detection rate is lower when the collapse is rapid with short duration as in this case. The collapse process for the Dot Com episode is much smoother, which partly explains the detective capability of the BSADF* test since the date of the bubble collapse clearly differs from the date of later market recovery. According to the BSADF* test, the crash lasts from February 2000 to December 2000, followed by a further correction in February 2004 and full return to normal market conditions in April 2004. As examples of rapid and smooth collapsing patterns, Figure 5 displays the enlarged graphs of the 1986-1987 bubble episode and the early 2000s crisis episodes.

Some sensitivity analyses are presented in the online supplement (Phillips and Shi, 2016). First, to address potential conditional heteroskedasticity in the data, a wild bootstrap is used to compute finite sample critical values. The results differ only marginally with the findings based on asymptotics without nonstationary volatility. Second, as an alternative lag order selection method, we use BIC with a maximum lag of four for each subsample regression. We find that the procedure based on BIC tends to provide timelier signals but at the cost of more false positives than the one based on a fixed lag order of unity. Finally, the PSY methods are applied to the log real Nasdaq price index as in PWY (2011), instead of the price-dividend ratio. Procedures based on the log real Nasdaq price index tend to provide an earlier but noisier signal of bubble existence. Use of this series can also lead to the identification of different bubble and crisis episodes, including crises that are not pre-dated by bubble behavior. This is not surprising as by conducting the test on the log price series alone there is no control for (or implied connection with) the market fundamentals. In addition, for comparison, we provide in the online supplement test results from application of PWY to the same data and with the exact settings of the tuning parameters (i.e. lag order, minimum window size). The PWY algorithm identifies the starting of the Dot Com episode eight months earlier (i.e. April 1996) but sets the collapse date three months later. PWY does not detect the 1983 episode.

The sub-martingale (and implied explosive autoregressive) property of bubbles considered in the theory literature on rational bubbles and by Diba and Grossman (1988) is fundamental to the bubble detection procedures of PWY, HB, PSY, and HLST and plays a key role in the definition of the expansionary phase of bubbles. Diba and Grossman (1988) argue that given free disposal a negative rational-bubble cannot exist as "stock holders cannot rationally expect a stock price to decrease without bound and, hence, to become negative at a finite future date." 
Figure 5: The 1986-1987 bubble episode and the early 2000s crisis episode

(a) The 1986-87 bubble episode

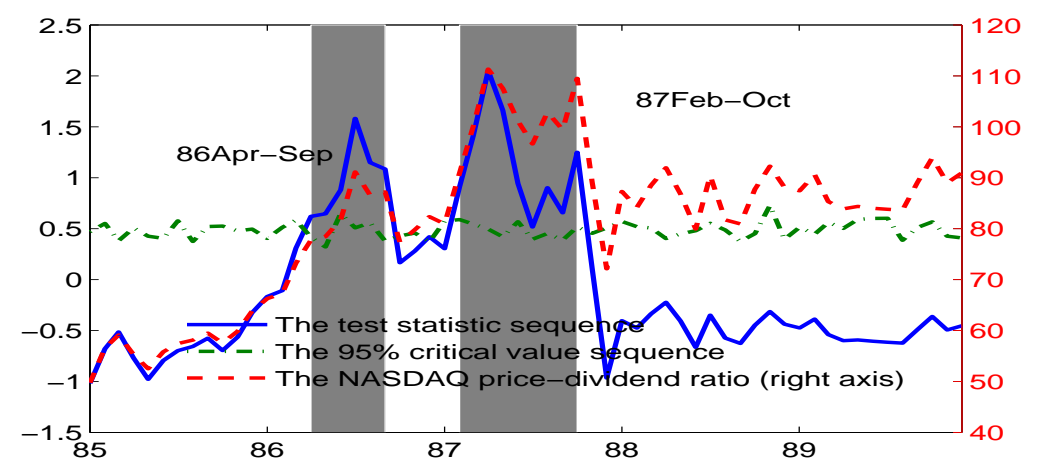

(b) The early 2000s crisis episode

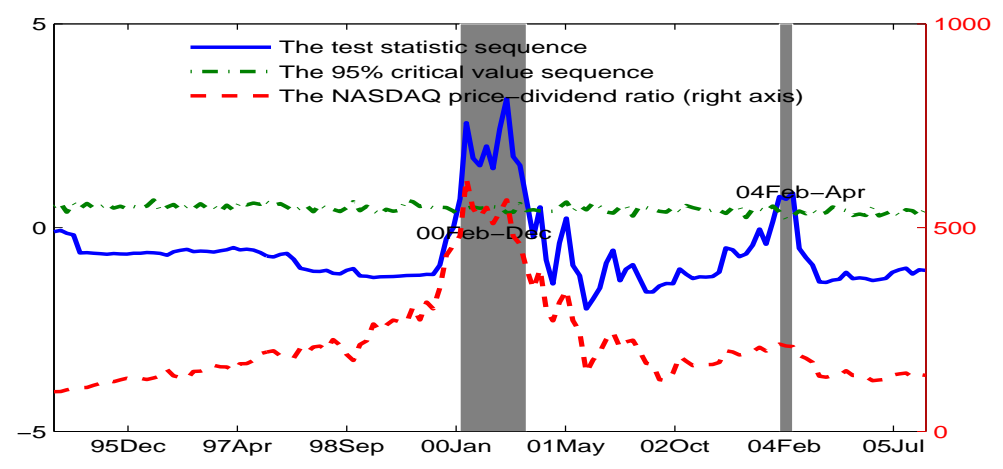

This is reflected in the sub-martingale property of bubbles and hence the aforementioned bubble detection techniques are primarily designed for positive bubble detection. This does not mean, of course, that short lived irrational negative bubbles do not occur. Recent work by Phillips (2016) gives behavioral economic theory that supports both expansionary and contractionary phases of bubbles.

Moreover, both the PWY and PSY tests can detect negative bubbles, especially when there are sharply falling markets. For example, PSY(2015a), Yiu, et al. (2013), Fantazzini (2016), and $\mathrm{Hu}$ and Oxley $(2017 \mathrm{a}, 2017 \mathrm{~b})$ have all found that these tests can register significance when prices fall rapidly. In fact, the tests can be shown to register significance whenever there are sustained strong directional movements in a trajectory (either positive or negative). Details of this capability and the associated asymptotic theory have been worked out in an ongoing study and will be reported in a later paper. Recognizing this capability, we here recommend users to identify ex-post whether test significance signals expansionary or contractionary phases. 


\section{Conclusion}

Financial bubbles are typically characterized by mildly explosive expansions and subsequent contractions that can be abrupt, extended, or various combinations of the two. In this work we have modeled financial contractions using a mildly integrated process that can capture a variety of forms of reversion to normal martingale behavior in a financial market. The resulting model has multiple break points corresponding to the bubble origination and peak, the implosion of the bubble, and the reversion to normality. This framework is intended to be more realistic than simpler models that assume an abrupt collapse of a bubble to normality and is therefore suited to a wider range of practical applications.

The limit theory shows that the bubble dating strategy of Phillips, Shi and Yu (2013a,b) delivers consistent date estimates within this more realistic bubble generating framework and simulations corroborate its advantages in finite samples.

The new reverse regression implementation of the PSY strategy developed here helps to detect crises and estimate their associated turning points. The strategy is suited to cases of a single crash or multiple crashes and provides consistent estimates of the origination, termination, and recovery dates of a crisis under certain conditions. The reverse regression procedure may be interpreted as an econometric foundation for the real-time technical analysis of corrections and crises in financial markets. Crisis detection methodology of this type can be applied when crises or corrections occur in seemingly normal periods that are not prefaced by an expansionary bubble phase. In applying these methods to the Nasdaq stock market over 1973-2013, the tests identify several crisis incidents, including the 1973 and 1976 stock market crashes and the famous 'black Monday' crash of 1987, in addition to the 1990s Nasdaq bubble episode.

\section{References}

Abreu, D. and Brunnermeier, M. K., 2003, Bubbles and crashes, Econometrica, 71, 173-204.

Akerlof, G. A. and Shiller, R. J., 2009, Animal Spirits: how human psychology drives the economy and why it matters for global capitalism. Princeton: Princeton University Press.

Bohl, M. T., Kaufmann, P., and Stephan, P. M., 2013, From hero to zero: Evidence of performance reversal and speculative bubbles in German renewable energy stocks. Energy Economics, 37: 40-51.

Blanchard, O. J.,1979, Speculative bubbles, crashes and rational expectations. Economics letters, 3(4), 387-389.

Chen, X., and Funke, M., 2013, Real-time warning signs of emerging and collapsing Chinese house price bubbles. National Institute Economic Review, 223(1), R39-R48.

Das, S., Gupta, R., and Kanda, P. T., 2011, International articles: Bubbles in south African house prices and their impact on consumption. Journal of Real Estate Literature, 19(1):6991. 
Diba, B.T., and Grossman, H.I., 1988, Explosive rational bubbles in stock prices? The American Economic Review, 78, 520-530.

Etienne, X., Irwin, S. H., and Garcia, P., 2013, Bubbles in food commodity markets: Four decades of evidence. Journal of International Money and Finance, 97(1):65-87.

Fantazzini, D., 2016, The oil price crash in 2014/15: Was there a (negative) financial bubble? Energy Policy, 96, 383-396.

Gutierrez, L., 2013, Speculative bubbles in agricultural commodity markets. European Review of Agricultural Economics, 40(2):217-238.

Harvey D. I., Leybourne S.J., Sollis R, 2015, Recursive right-tailed unit root tests for an explosive asset price bubble. Journal of Financial Econometrics, 13, 166-187.

Harvey, D. I., S. J. Leybourne, R. Sollis, and R. A. Taylor, 2016, Tests for explosive financial bubbles in the presence of non-stationary volatility. Journal of Empirical Finance, 38, 548-574.

Homm, U., and Breitung, J., 2012, Testing for speculative bubbles in stock markets: a comparison of alternative methods. Journal of Financial Econometrics, 10(1):198-231.

Hu, Y., and Oxley, L., 2017a, Exuberance, bubbles or froth: Some historical results using long run house data for Amsterdam, Norway, and Paris. Working Paper, University of Waikato.

Hu, Y., and Oxley, L., 2017b, Exuberance in historical stock prices during the Mississippi bubble and South Sea bubble episodes. Working Paper, University of Waikato.

Huang, W., Zheng, H., and Chia, W. M., 2010, Financial crises and interacting heterogeneous agents. Journal of Economic Dynamics and Control, 34(6), 1105-1122.

Li, W. and Xue, H., 2009, A Bayesian's Bubble. The Journal of Finance, 64(6), 2665-2701.

Kindleberger, C. P., and Aliber, R. Z., 2005, Manias, Panics and Crashes; A History of Financial Crises, Hoboken, New Jersey: John Wiley and Sons, Inc.

Leybourne, S., Kim T-H., and A. M. R. Taylor, 2007, "Detecting multiple changes in persistence," Studies in Nonlinear Dynamics and Econometrics, 11, 1-32.

Meyer, G., 2013, Commodity booms, busts and bubbles. Financial Times, March 25.

Pavlidis, E., Paya, I., and Peel, D.,2015, A new test for rational speculative bubbles using forward exchange rates: The case of the interwar german hyperinflation, Working Paper, The Department of Economics, Lancaster.

Phillips, P. C. B., 1987, Time series regression with a unit root. Econometrica, 55(2):277-301.

Phillips, P. C. B., 2016, Modeling speculative bubbles with diverse investor expectations. Research in Economics, 70(3): 375-387. 
Phillips, P. C. B., and Magdalinos, T., 2007a, Limit theory for moderate deviations from a unit root. Journal of Econometrics, 136(1):115-130.

Phillips, P. C. B. and Magdalinos, T., 2007b, Limit Theory for Moderate Deviations from Unity under Weak Dependence" in G. D. A. Phillips and E. Tzavalis (Eds.) The Refinement of Econometric Estimation and Test Procedures: Finite Sample and Asymptotic Analysis. Cambridge: Cambridge University Press, 2007, pp.123-162.

Phillips, P. C. B., and Magdalinos, T., 2009. Unit root and cointegrating limit theory when initialization is in the infinite past. Econometric Theory, 25, 1682-1715.

Phillips, P. C. B., and Perron, P., 1988, Testing for a unit root in time series regression. Biometrika, 75(2):335-346.

Phillips, P. C. B., and Shi, S., 2016, Supplementary material to 'Financial Bubble Implosion and Reverse Regression'. Econometric Theory Supplemental Material (typesetter to provide the web address)

Phillips, P. C., Shi, S., and Yu, J., 2011, Specification Sensitivity in Right-Tailed Unit Root Testing for Explosive Behavior. Singapore Management University Economics and Statistics Working Paper Series, No. 22-2011.

Phillips, P. C. B., Shi, S., and Yu, J., 2014, Specification sensitivity in right-tailed unit root testing for explosive behaviour. Oxford Bulletin of Economics and Statistics, Vol. 76, No. 3, pp. 315-333.

Phillips, P. C. B., Shi, S., and Yu, J., 2015a, Testing for multiple bubbles: Historical episodes of exuberance and collapse in the S\&P 500. International Economic Review, 56(4):1043-1078.

Phillips, P. C. B., Shi, S., and Yu, J., 2015b, Testing for multiple bubbles: Limit Theory of Real Time Detectors. International Economic Review, 56(4):1079-1134.

Phillips, P. C. B., Wu, Y., and Yu, J., 2011, Explosive behavior in the 1990s NASDAQ: When did exuberance escalate asset values? International Economic Review, 52(1):201-226.

Phillips, P. C. B, and Yu, J., 2011a, Warning Signs of Future Asset Bubbles, The Straits Times, Singapore, April 26.

Phillips, P. C. B., and Yu, J., 2011b, Dating the timeline of financial bubbles during the subprime crisis. Quantitative Economics, 2(3):455-491.

Phillips, P. C. B, and Yu, J., 2013, Bubble or Roller Coaster in World Stock Markets, The Business Times, Singapore, June 28.

Rosser, J. B., 2000, From Catastrophe to Chaos: A General Theory of Economic Discontinuities: Mathematics, Microeconomics and Finance, Vol 1. Springer. 
Shi, S., and Song, Y., 2012, Identifying speculative bubbles with an in finite hidden Markov model, Journal of Financial Econometrics, 14(1):159-184.

Yiu, M., Yu, J., and Jin, L., 2013, Detecting Bubbles in the Hong Kong Residential Property Market: An Explosive-Pattern Approach. Journal of Asian Economics, 28(1):115-124. 


\section{Appendix: Limit Theory and Proofs of Main Results}

This Appendix has two main sections (A and B). The first provides limit theory for the BSDF* statistic under the null based on the reverse regression model formulation (8). The second gives limit theory for the BSDF and BSDF* statistics under the alternative. Supporting lemmas are stated here. Complete details of the derivations and the supporting lemmas are given in Phillips and Shi (2016) which provides a full technical supplement to the paper. The general framework of the derivations follows PSY (2015a\&b) but important differences arise because of the treatment of the reverse regression statistic, the endogeneity involved in that regression, and the presence of an asymptotically negligible drift in the time series.

\section{A The limit behaviour of the $\mathrm{BSDF}^{*}$ statistic under the null}

Lemmas A1 and A2 below provide some standard partial sum asymptotics that hold under the following assumption, where the input process $\varepsilon_{t}$ is assumed to be iid for convenience but may be extended to martingale differences with appropriate changes to the limit theory. These results mirror those given in PSY (2015b).

Assumption (EC) Let $u_{t}=\psi(L) \varepsilon_{t}=\Sigma_{j=0}^{\infty} \psi_{j} \varepsilon_{t-j}$, where $\Sigma_{j=0}^{\infty} j\left|\psi_{j}\right|<\infty$ and $\left\{\varepsilon_{t}\right\}$ is an i.i.d sequence with mean zero, variance $\sigma^{2}$ and finite fourth moment.

Lemma A.1 Suppose $u_{t}$ satisfies error condition EC. Define $M_{T}(g)=1 / T \sum_{s=1}^{[T g]} u_{s}$ with $r \in$ $\left[g_{0}, 1\right]$ and $\xi_{t}=\sum_{s=1}^{t} u_{s}$. Let $g_{2}, g_{w} \in\left[g_{0}, 1\right]$ and $g_{1}=g_{2}-g_{w}$. The following hold:

(1) $\sum_{s=1}^{t} u_{s}=\psi(1) \sum_{s=1}^{t} \varepsilon_{s}+\eta_{t}-\eta_{0}$, where $\eta_{t}=\sum_{j=0}^{\infty} \alpha_{j} \varepsilon_{t-j}, \eta_{0}=\sum_{j=0}^{\infty} \alpha_{j} \varepsilon_{-j}$ and $\alpha_{j}=-\sum_{i=1}^{\infty} \psi_{j+i}$, which is absolutely summable.

(2) $\frac{1}{T} \sum_{t=\left\lfloor T g_{1}\right\rfloor}^{\left\lfloor T g_{2}\right\rfloor} \varepsilon_{t}^{2} \stackrel{p}{\rightarrow} \sigma^{2} g_{w}$.

(3) $T^{-1 / 2} \sum_{t=1}^{[T g]} \varepsilon_{t} \stackrel{L}{\rightarrow} \sigma W(g)$.

(4) $T^{-1} \sum_{t=\left\lfloor T g_{1}\right\rfloor}^{\left\lfloor T g_{2}\right\rfloor} \sum_{s=1}^{t-1} \varepsilon_{s} \varepsilon_{t} \stackrel{L}{\rightarrow} \sigma^{2}\left[\int_{g_{1}}^{g_{2}} W(s) d W-\frac{1}{2} g_{w}\right]$.

(5) $T^{-3 / 2} \sum_{t=\left\lfloor T g_{1}\right\rfloor}^{\left\lfloor T g_{2}\right\rfloor} \varepsilon_{t} t \stackrel{L}{\rightarrow} \sigma\left[g_{2} W\left(g_{2}\right)-g_{1} W\left(g_{1}\right)-\int_{g_{1}}^{g_{2}} W(s) d s\right]$.

(6) $T^{-1} \sum_{t=\left\lfloor T g_{1}\right\rfloor}^{\left\lfloor T g_{2}\right\rfloor}\left(\eta_{t-1}-\eta_{0}\right) \varepsilon_{t} \stackrel{p}{\rightarrow} 0$.

(7) $T^{-1 / 2}\left(\eta_{[T g]}-\eta_{0}\right) \stackrel{p}{\rightarrow} 0$.

(8) $\sqrt{T} M_{T}(g) \stackrel{L}{\rightarrow} \psi(1) \sigma W(g)$.

(9) $T^{-3 / 2} \sum_{t=\left\lfloor T g_{1}\right\rfloor}^{\left\lfloor T g_{2}\right\rfloor} \xi_{t-1} \stackrel{L}{\rightarrow} \psi(1) \sigma \int_{g_{1}}^{g_{2}} W(s) d s$.

(10) $T^{-5 / 2} \sum_{t=\left\lfloor T g_{1}\right\rfloor}^{\left\lfloor T g_{2}\right\rfloor} \xi_{t-1} t \stackrel{L}{\rightarrow} \psi(1) \sigma \int_{g_{1}}^{g_{2}} W(s) s d s$.

(11) $T^{-2} \sum_{t=\left\lfloor T g_{1}\right\rfloor}^{\left\lfloor T g_{2}\right\rfloor} \xi_{t-1}^{2} \stackrel{L}{\rightarrow} \sigma^{2} \psi(1)^{2} \int_{g_{1}}^{g_{2}} W(s)^{2} d s$.

(12) $T^{-3 / 2} \sum_{t=\left\lfloor T g_{1}\right\rfloor}^{\left\lfloor T g_{2}\right\rfloor} \xi_{t} \varepsilon_{t-j} \stackrel{p}{\rightarrow} 0, \forall j=0, \pm 1, \pm 2, \ldots$ 
Lemma A.2 Define $X_{t}^{*}=-\alpha_{T} \psi(1) t+\sum_{s=1}^{t} \omega_{s}$, where $\alpha_{T}=c T^{-\eta}$ with $\eta>1 / 2$ and $\omega_{t}=$ $-u_{T+2-t}=\psi(L) v_{t}$. Let $u_{t}$ satisfy condition EC. Then

$$
\begin{aligned}
& \text { (a) } T^{-1} \sum_{t=\left\lfloor T g_{1}\right\rfloor}^{\left\lfloor T g_{2}\right\rfloor} X_{t-1}^{*} v_{t} \stackrel{L}{\rightarrow} \psi(1) \sigma^{2}\left[\int_{1-g_{2}}^{1-g_{1}} W(s) d W+\frac{1}{2} g_{w}\right] . \\
& \text { (b) } T^{-3 / 2} \sum_{t=\left\lfloor T g_{1}\right\rfloor}^{\left\lfloor T g_{2}\right\rfloor} X_{t-1}^{*} \stackrel{L}{\rightarrow} \psi(1) \sigma \int_{1-g_{2}}^{1-g_{1}} W(s) d s . \\
& \text { (c) } T^{-2} \sum_{t=\left\lfloor T g_{1}\right\rfloor}^{\left\lfloor T g_{2}\right\rfloor} X_{t-1}^{* 2} \stackrel{L}{\rightarrow} \sigma^{2} \psi(1)^{2} \int_{1-g_{2}}^{1-g_{1}} W(s)^{2} d s . \\
& \text { (d) } T^{-3 / 2} \sum_{t=\left\lfloor T g_{1}\right\rfloor}^{\left\lfloor T g_{2}\right\rfloor} X_{t-1}^{*} v_{t-j} \stackrel{p}{\rightarrow} 0, \quad j=0,1, \cdots \\
& \text { (e) } T^{-1 / 2} \sum_{t=\left\lfloor T g_{1}\right\rfloor}^{\left\lfloor T g_{2}\right\rfloor} v_{t} \stackrel{L}{\rightarrow}-\sigma \int_{1-g_{2}}^{1-g_{1}} d W(s) .
\end{aligned}
$$

With these results and using standard weak convergence methods, we can derive the asymptotic distribution of the $B S D F_{g}^{*}\left(g_{0}\right)$ statistic given in Theorem 3.1. A complete proof of Theorem 3.1 is provided in the technical supplement (PS, 2014).

\section{B The limit behaviour of the BSDF statistic under the Alterna- tive}

\section{B.1 Notation}

- The bubble period $B=\left[T_{e}, T_{c}\right]$, where $T_{e}=\left\lfloor T f_{e}\right\rfloor$ and $T_{c}=\left\lfloor T f_{c}\right\rfloor$.

- The crisis period $C=\left(T_{c}, T_{r}\right]$, where $T_{r}=\left\lfloor T f_{r}\right\rfloor$.

- The normal market periods $N_{0}=\left[1, T_{e}\right)$ and $N_{1}=\left[T_{r}+1, T\right]$, where $T$ is the last observation of the sample.

- The data generating process is specified as

$$
X_{t}=\left\{\begin{array}{cc}
c T^{-\eta}+X_{t-1}+\varepsilon_{t}, \text { constant } c, \eta>1 / 2, & t \in N_{0} \cup N_{1}, \\
\delta_{T} X_{t-1}+\varepsilon_{t}, & t \in B \\
\gamma_{T} X_{t-1}+\varepsilon_{t}, & t \in C
\end{array},\right.
$$

where $\varepsilon_{t} \sim N\left(0, \sigma^{2}\right), X_{0}=O_{p}(1), \delta_{T}=1+c_{1} T^{-\alpha}$ and $\gamma_{T}=1-c_{2} T^{-\beta}$ with $c_{1}, c_{2}>0$ and $\alpha, \beta \in[0,1)$. If $\alpha>\beta$, the rate of bubble collapse is faster than that of the bubble expansion. If $\alpha<\beta$, the rate of bubble collapse is slower than that of the bubble expansion. 
- Let $X_{t}^{*}=X_{T+1-t}$. The dynamics of $X_{t}^{*}$ are

$$
X_{t}^{*}=\left\{\begin{array}{ll}
-c T^{-\eta}+X_{t-1}^{*}+v_{t}, \text { constant } c, \eta>1 / 2, & t \in N_{0} \cup N_{1} \\
\gamma_{T}^{-1} X_{t-1}^{*}+\gamma_{T}^{-1} v_{t}, & t \in C \\
\delta_{T}^{-1}\left[X_{t-1}^{*}+v_{t}\right], & t \in B
\end{array},\right.
$$

where $v_{t}=-\varepsilon_{T+2-t} \sim N\left(0, \sigma^{2}\right)$ and $X_{0}^{*}=X_{T+1}$.

- Let $\tau_{1}=\left\lfloor T g_{1}\right\rfloor$ and $\tau_{2}=\left\lfloor T g_{2}\right\rfloor$ be the start and end points of the regression. We have $T_{1}=T+1-\tau_{2}, T_{2}=T+1-\tau_{1}$ and $\tau_{w}=\left\lfloor T g_{w}\right\rfloor$ be the regression window size.

- Let $\tau_{e}=\left\lfloor T g_{e}\right\rfloor, \tau_{r}=\left\lfloor T g_{r}\right\rfloor$, and $\tau_{c}=\left\lfloor T g_{c}\right\rfloor$, where $g_{e}=1-f_{r}, g_{c}=1-f_{c}, g_{r}=1-f_{e}$. This suggests that $N_{1}=\left[1, \tau_{e}\right), C=\left[\tau_{e}, \tau_{c}\right), B=\left[\tau_{c}, \tau_{r}\right], N_{0}=\left(\tau_{r}, T\right]$.

\section{B.2 Dating Bubble Expansion}

Lemma B.1 Under the data generating process (13):

(1) For $t \in N_{0}, X_{t=\lfloor T p\rfloor} \sim_{a} T^{1 / 2} B(p)$.

(2) For $t \in B, X_{t=\lfloor T p\rfloor}=\delta_{T}^{t-T_{e}} X_{T_{e}}\left\{1+o_{p}(1)\right\} \sim_{a} T^{1 / 2} \delta_{T}^{t-T_{e}} B\left(f_{e}\right)$.

(3) For $t \in C$,

$$
X_{t=\lfloor T p\rfloor}=\gamma_{T}^{t-T_{c}} X_{T_{c}}+\sum_{j=0}^{t-T_{c}-1} \gamma_{T}^{j} \varepsilon_{t-j} \sim_{a} T^{1 / 2} \delta_{T}^{T_{c}-T_{e}} \gamma_{T}^{t-T_{c}} B\left(f_{e}\right)+T^{\beta / 2} X_{c_{2}}
$$

(4) For $t \in N_{1}$,

$$
X_{t=\lfloor T p\rfloor}=\left\{\begin{array}{ll}
\sum_{j=0}^{t-T_{r}-1} \varepsilon_{t-j}\left\{1+o_{p}(1)\right\} \sim_{a} T^{1 / 2}\left[B(p)-B\left(f_{r}\right)\right] & \text { if } \alpha>\beta \\
\gamma_{T}^{T_{r}-T_{c}} X_{T_{c}} \sim_{a} T^{1 / 2} \gamma_{T}^{T_{r}-T_{c}} \delta_{T}^{T_{c}-T_{e}} B\left(f_{e}\right) & \text { if } \alpha<\beta
\end{array} .\right.
$$

Lemma B.2 Under the data generating process (13):

(1) For $T_{1} \in N_{0}$ and $T_{2} \in B$,

$$
\frac{1}{T_{w}} \sum_{j=T_{1}}^{T_{2}} X_{j}=\frac{T^{\alpha} \delta_{T}^{T_{2}-T_{e}}}{T_{w} c_{1}} X_{T_{e}}\left\{1+o_{p}(1)\right\} \sim_{a} T^{\alpha-1 / 2} \delta_{T}^{T_{2}-T_{e}} \frac{1}{f_{w} c_{1}} B\left(f_{e}\right) .
$$

(2) For $T_{1} \in N_{0}$ and $T_{2} \in C$,

$$
\frac{1}{T_{w}} \sum_{j=T_{1}}^{T_{2}} X_{j}=\left\{\begin{array}{ll}
\frac{T^{\alpha} \delta_{T}^{T_{c}-T_{e}}}{T_{w} c_{1}} X_{T_{e}}\left\{1+o_{p}(1)\right\} \sim_{a} T^{\alpha-1 / 2} \delta_{T}^{T_{c}-T_{e}} \frac{1}{f_{w} c_{1}} B\left(f_{e}\right) & \text { if } \alpha>\beta \\
X_{T_{c}} \frac{T^{\beta}}{T_{w} c_{2}}\left\{1+o_{p}(1)\right\} \sim_{a} T^{\beta-1 / 2} \delta_{T}^{T_{c}-T_{e}} \frac{B\left(f_{e}\right)}{f_{w} c_{2}} & \text { if } \alpha<\beta
\end{array} .\right.
$$

(3) For $T_{1} \in N_{0}$ and $T_{2} \in N_{1}$,

$$
\frac{1}{T_{w}} \sum_{j=T_{1}}^{T_{2}} X_{j}=\left\{\begin{array}{ll}
\frac{T^{\alpha} \delta_{T}^{T_{c}-T_{e}}}{T_{w} c_{1}} X_{T_{e}}\left\{1+o_{p}(1)\right\} \sim_{a} T^{\alpha-1 / 2} \delta_{T}^{T_{c}-T_{e}} \frac{1}{f_{w} c_{1}} B\left(f_{e}\right) & \text { if } \alpha>\beta \\
\frac{T^{\beta}}{T_{w} c_{2}} X_{T_{c}}\left\{1+o_{p}(1)\right\} \sim_{a} T^{\beta-1 / 2} \delta_{T}^{T_{c}-T_{e}} \frac{1}{f_{w} c_{2}} B\left(f_{e}\right) & \text { if } \alpha<\beta
\end{array} .\right.
$$


(4) For $T_{1} \in B$ and $T_{2} \in C$,

$$
\frac{1}{T_{w}} \sum_{j=T_{1}}^{T_{2}} X_{j}=\left\{\begin{array}{ll}
\frac{T^{\alpha} \delta_{T}^{T_{c}-T_{e}}}{T_{w} c_{1}} X_{T_{e}}\left\{1+o_{p}(1)\right\} \sim_{a} T^{\alpha-1 / 2} \delta_{T}^{T_{c}-T_{e}} \frac{1}{f_{w} c_{1}} B\left(f_{e}\right) & \text { if } \alpha>\beta \\
X_{T_{c}} \frac{T^{\beta}}{T_{w} c_{2}}\left\{1+o_{p}(1)\right\} \sim_{a} T^{\beta-1 / 2} \delta_{T}^{T_{c}-T_{e}} \frac{1}{f_{w} c_{2}} B\left(f_{e}\right) & \text { if } \alpha<\beta
\end{array} .\right.
$$

(5) For $T_{1} \in B$ and $T_{2} \in N_{1}$,

$$
\frac{1}{T_{w}} \sum_{j=T_{1}}^{T_{2}} X_{j}=\left\{\begin{array}{ll}
\frac{T^{\alpha} \delta_{T}^{T_{c}-T_{1}}}{T_{w} c_{1}} X_{T_{e}}\left\{1+o_{p}(1)\right\} \sim_{a} T^{\alpha-1 / 2} \delta_{T}^{T_{c}-T_{e}} \frac{1}{f_{w} c_{1}} B\left(f_{e}\right) & \text { if } \alpha>\beta \\
X_{T_{c}} \frac{T^{\beta}}{T_{w} c_{2}}\left\{1+o_{p}(1)\right\} \sim_{a} T^{\beta-1 / 2} \delta_{T}^{T_{c}-T_{e}} \frac{1}{f_{w} c_{2}} B\left(f_{e}\right) & \text { if } \alpha<\beta
\end{array} .\right.
$$

(6) For $T_{1} \in C$ and $T_{2} \in N_{1}$,

$$
\frac{1}{T_{w}} \sum_{j=T_{1}}^{T_{2}} X_{j}=\left\{\begin{array}{ll}
\frac{1}{T_{w}} \sum_{j=T_{r}+1}^{T_{2}} \sum_{i=0}^{j-T_{r}-1} \varepsilon_{j-i}\left\{1+o_{p}(1)\right\} \sim_{a} T^{1 / 2} \frac{f_{2}-f_{r}}{f_{w}} \int_{f_{r}}^{f_{2}}\left[B(s)-B\left(f_{r}\right)\right] d s & \text { if } \alpha>\beta \\
X_{T_{c}} \frac{\gamma_{T}^{T_{1}-T_{c}} T^{\beta}}{T_{w} c_{2}}\left\{1+o_{p}(1)\right\} \sim_{a} T^{\beta-1 / 2} \delta_{T}^{T_{c}-T_{e}} \gamma_{T}^{T_{1}-T_{c}} \frac{1}{c_{2} f_{w}} B\left(f_{e}\right) & \text { if } \alpha<\beta
\end{array} .\right.
$$

Lemma B.3 Define the centered quantity $\tilde{X}_{t}=X_{t}-T_{w}^{-1} \sum_{j=T_{1}}^{T_{2}} X_{j}$.

(1) For $T_{1} \in N_{0}$ and $T_{2} \in B$,

$$
\tilde{X}_{t}=\left\{\begin{array}{ll}
-\frac{T^{\alpha} \delta_{T}^{T_{2}-T_{e}}}{T_{w} c_{1}} X_{T_{e}}\left\{1+o_{p}(1)\right\} & \text { if } t \in N_{0} \\
{\left[\delta_{T}^{t-T_{e}}-\frac{T^{\alpha} \delta_{T}^{T_{2}}-T_{e}}{T_{w} c_{1}}\right] X_{T_{e}}\left\{1+o_{p}(1)\right\}} & \text { if } t \in B
\end{array} .\right.
$$

(2) For $T_{1} \in N_{0}$ and $T_{2} \in C$, if $\alpha>\beta$

$$
\tilde{X}_{t}= \begin{cases}-\frac{T^{\alpha} \delta_{T}^{T_{c}-T_{e}}}{T_{w} c_{1}} X_{T_{e}}\left\{1+o_{p}(1)\right\} & \text { if } t \in N_{0} \\ {\left[\delta_{T}^{t-T_{e}}-\frac{T^{\alpha} \delta_{T}^{T_{c}}-T_{e}}{T_{w} c_{1}}\right] X_{T_{e}}\left\{1+o_{p}(1)\right\}} & \text { if } t \in B \\ {\left[\gamma_{T}^{t-T_{c}} X_{T_{c}}-\frac{T^{\alpha} \delta_{T}^{T_{c}-T_{e}}}{T_{w} c_{1}} X_{T_{e}}\right]\left\{1+o_{p}(1)\right\}} & \text { if } t \in C\end{cases}
$$

and if $\alpha<\beta$,

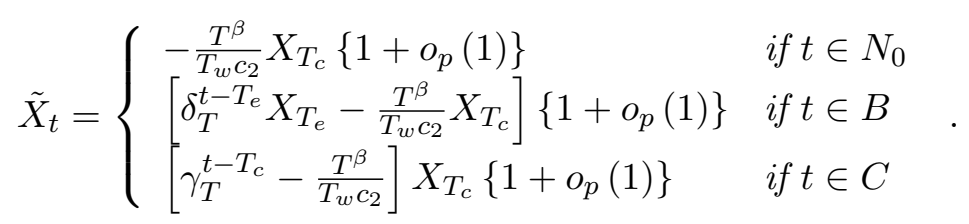

(3) For $T_{1} \in N_{0}$ and $T_{2} \in N_{1}$, if $\alpha>\beta$

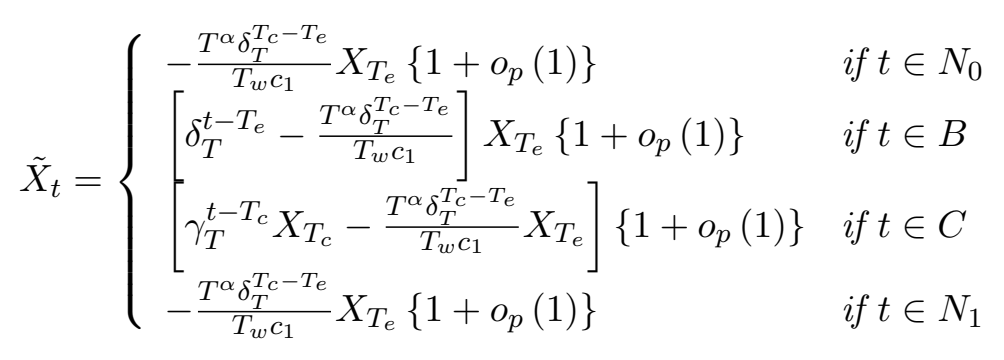


and if $\alpha<\beta$,

$$
\tilde{X}_{t}=\left\{\begin{array}{ll}
-\frac{T^{\beta}}{T_{w} c_{2}} X_{T_{c}}\left\{1+o_{p}(1)\right\} & \text { if } t \in N_{0} \\
{\left[\delta_{T}^{t-T_{e}} X_{T_{e}}-\frac{T^{\beta}}{T_{w} c_{2}} X_{T_{c}}\right]\left\{1+o_{p}(1)\right\}} & \text { if } t \in B \\
\left.\gamma_{T}^{t-T_{c}}-\frac{T^{\beta}}{T_{w} c_{2}}\right] X_{T_{c}}\left\{1+o_{p}(1)\right\} & \text { if } t \in C \\
-\frac{T^{\beta}}{T_{w} c_{2}} X_{T_{c}}\left\{1+o_{p}(1)\right\} & \text { if } t \in N_{1}
\end{array} .\right.
$$

(4) For $T_{1} \in B$ and $T_{2} \in C$, if $\alpha>\beta$

$$
\tilde{X}_{t}= \begin{cases}{\left[\delta_{T}^{t-T_{e}}-\frac{T^{\alpha} \delta_{T}^{T_{c}}-T_{e}}{T_{w} c_{1}}\right] X_{T_{e}}\left\{1+o_{p}(1)\right\}} & \text { if } t \in B \\ \left.\gamma_{T}^{t-T_{c}} X_{T_{c}}-\frac{T^{\alpha} \delta_{T}^{T_{c}-T_{e}}}{T_{w} c_{1}} X_{T_{e}}\right]\left\{1+o_{p}(1)\right\} & \text { if } t \in C\end{cases}
$$

and if $\alpha<\beta$,

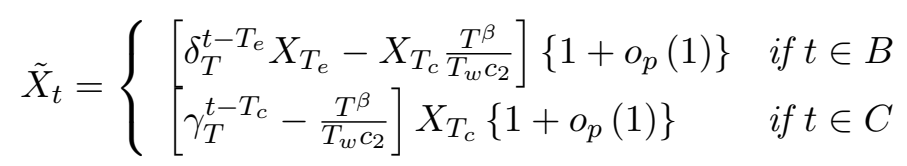

(5) For $T_{1} \in B$ and $T_{2} \in N_{1}$, if $\alpha>\beta$

$$
\tilde{X}_{t}= \begin{cases}{\left[\delta_{T}^{t-T_{e}}-\frac{T^{\alpha} \delta_{T}^{T_{c}-T_{e}}}{T_{w} c_{1}}\right] X_{T_{e}}\left\{1+o_{p}(1)\right\}} & \text { if } t \in B \\ \left.\gamma_{T}^{t-T_{c}} X_{T_{c}}-\frac{T^{\alpha} \delta_{T}^{T_{c}-T_{e}}}{T_{w} c_{1}} X_{T_{e}}\right]\left\{1+o_{p}(1)\right\} & \text { if } t \in C \\ -\frac{T^{\alpha} \delta_{T}^{T_{c}-T_{e}}}{T_{w} c_{1}} X_{T_{e}}\left\{1+o_{p}(1)\right\} & \text { if } t \in N_{1}\end{cases}
$$

and if $\alpha<\beta$,

$$
\tilde{X}_{t}= \begin{cases}{\left[\delta_{T}^{t-T_{e}} X_{T_{e}}-\frac{T^{\beta}}{T_{w} c_{2}} X_{T_{c}}\right]\left\{1+o_{p}(1)\right\}} & \text { if } t \in B \\ \left.\gamma_{T}^{t-T_{c}}-\frac{T^{\beta}}{T_{w} c_{2}}\right] X_{T_{c}}\left\{1+o_{p}(1)\right\} & \text { if } t \in C \\ -\frac{T^{\beta}}{T_{w} c_{2}} X_{T_{c}}\left\{1+o_{p}(1)\right\} & \text { if } t \in N_{1}\end{cases}
$$

(6) For $T_{1} \in C$ and $T_{2} \in N_{1}$, if $\alpha>\beta$,

$$
\tilde{X}_{t}= \begin{cases}{\left[\gamma_{T}^{t-T_{c}} X_{T_{c}}-\frac{1}{T_{w}} \sum_{j=T_{r}+1}^{T_{2}} \sum_{i=0}^{j-T_{r}-1} \varepsilon_{j-i}\right]\left\{1+o_{p}(1)\right\}} & \text { if } t \in C \\ {\left[\sum_{j=0}^{t-T_{r}-1} \varepsilon_{t-j}-\frac{1}{T_{w}} \sum_{j=T_{r}+1}^{T_{2}} \sum_{i=0}^{j-T_{r}-1} \varepsilon_{j-i}\right]\left\{1+o_{p}(1)\right\}} & \text { if } t \in N_{1}\end{cases}
$$

and if $\alpha<\beta$,

$$
\tilde{X}_{t}=\left\{\begin{array}{ll}
{\left[\gamma_{T}^{t-T_{c}}-\frac{\gamma_{T}^{T_{1}-T_{c}} T^{\beta}}{T_{w} c_{2}}\right] X_{T_{c}}\left\{1+o_{p}(1)\right\}} & \text { if } t \in C \\
-\frac{\gamma_{T}^{T_{1}-T_{c}} T^{\beta}}{T_{w} T_{2}} X_{T_{c}}\left\{1+o_{p}(1)\right\} & \text { if } t \in N_{1}
\end{array} .\right.
$$

Lemma B.4 Quadratic terms in $\tilde{X}_{t}$ behave as follows. 
(1) For $T_{1} \in N_{0}$ and $T_{2} \in B$,

$$
\sum_{j=T_{1}}^{T_{2}} \tilde{X}_{j-1}^{2} \sim_{a} T^{1+\alpha} \frac{\delta_{T}^{2\left(T_{2}-T_{e}\right)}}{2 c_{1}} B\left(f_{e}\right)^{2}
$$

(2) For $T_{1} \in N_{0}$ and $T_{2} \in C$,

$$
\sum_{j=T_{1}}^{T_{2}} \tilde{X}_{j-1}^{2} \sim_{a}\left\{\begin{array}{ll}
T^{1+\alpha} \delta_{T}^{2\left(T_{c}-T_{e}\right)} \frac{1}{2 c_{1}} B\left(f_{e}\right)^{2} & \text { if } \alpha>\beta \\
T^{1+\beta} \delta_{T}^{2\left(T_{c}-T_{e}\right)} \frac{1}{2 c_{2}} B\left(f_{e}\right)^{2} & \text { if } \alpha<\beta
\end{array} .\right.
$$

(3) For $T_{1} \in N_{0}$ and $T_{2} \in N_{1}$,

$$
\sum_{j=T_{1}}^{T_{2}} \tilde{X}_{j-1}^{2} \sim_{a}\left\{\begin{array}{ll}
T^{1+\alpha} \delta_{T}^{2\left(T_{c}-T_{e}\right)} \frac{1}{2 c_{1}} B\left(f_{e}\right)^{2} & \text { if } \alpha>\beta \\
T^{1+\beta} \delta_{T}^{2\left(T_{c}-T_{e}\right)} \frac{1}{2 c_{2}} B\left(f_{e}\right)^{2} & \text { if } \alpha \leq \beta
\end{array} .\right.
$$

(4) For $T_{1} \in B$ and $T_{2} \in C$,

$$
\sum_{j=T_{1}}^{T_{2}} \tilde{X}_{j-1}^{2} \sim_{a}\left\{\begin{array}{ll}
T^{1+\alpha} \delta_{T}^{2\left(T_{c}-T_{e}\right)} \frac{1}{2 c_{1}} B\left(f_{e}\right)^{2} & \text { if } \alpha>\beta \\
T^{1+\beta} \delta_{T}^{2\left(T_{c}-T_{e}\right)} \frac{1}{2 c_{2}} B\left(f_{e}\right)^{2} & \text { if } \alpha<\beta
\end{array} .\right.
$$

(5) For $T_{1} \in B$ and $T_{2} \in N_{1}$,

$$
\sum_{j=T_{1}}^{T_{2}} \tilde{X}_{j-1}^{2} \sim_{a}\left\{\begin{array}{ll}
T^{1+\alpha} \delta_{T}^{2\left(T_{c}-T_{e}\right)} \frac{1}{2 c_{1}} B\left(f_{e}\right)^{2} & \text { if } \alpha>\beta \\
T^{1+\beta} \delta_{T}^{2\left(T_{c}-T_{e}\right)} \frac{1}{2 c_{2}} B\left(f_{e}\right)^{2} & \text { if } \alpha<\beta
\end{array} .\right.
$$

(6) For $T_{1} \in C$ and $T_{2} \in N_{1}$,

$$
\sum_{j=T_{1}}^{T_{2}} \tilde{X}_{j-1}^{2} \sim_{a}\left\{\begin{array}{ll}
T^{2}\left(f_{2}-f_{r}\right)\left\{\int_{f_{r}}^{f_{2}}\left[B(s)-B\left(f_{r}\right)\right]^{2} d s-\frac{f_{2}-f_{r}}{f_{w}}\left[\int_{f_{r}}^{f_{2}}\left[B(s)-B\left(f_{r}\right)\right] d s\right]^{2}\right\} & \text { if } \alpha>\beta \\
T^{1+\beta} \delta_{T}^{2\left(T_{c}-T_{e}\right)} \gamma_{T}^{2\left(T_{1}-T_{c}\right)} \frac{1}{2 c_{2}} B\left(f_{e}\right)^{2} & \text { if } \alpha<\beta
\end{array} .\right.
$$

Lemma B.5 Cross-product terms involving $\tilde{X}_{t}$ and $\varepsilon_{t}$ behave as follows.

(1) For $T_{1} \in N_{0}$ and $T_{2} \in B$,

$$
\sum_{j=T_{1}}^{T_{2}} \tilde{X}_{j-1} \varepsilon_{j} \sim_{a} T^{(1+\alpha) / 2} \delta_{T}^{T_{2}-T_{e}} X_{c_{1}} B\left(f_{e}\right) .
$$

(2) For $T_{1} \in N_{0}$ and $T_{2} \in C$,

$$
\sum_{j=T_{1}}^{T_{2}} \tilde{X}_{j-1} \varepsilon_{j} \sim_{a} \begin{cases}T^{(1+\alpha) / 2} \delta_{T}^{T_{c}-T_{e}} B\left(f_{e}\right) X_{c_{1}} & \text { if } \alpha>\beta \\ T^{(1+\beta) / 2} \delta_{T}^{T_{c}-T_{e}} B\left(f_{e}\right) X_{c_{2}} & \text { if } \alpha<\beta\end{cases}
$$


(3) For $T_{1} \in N_{0}$ and $T_{2} \in N_{1}$,

$$
\sum_{j=T_{1}}^{T_{2}} \tilde{X}_{j-1} \varepsilon_{j} \sim_{a}\left\{\begin{array}{ll}
T^{(1+\alpha) / 2} \delta_{T}^{T_{c}-T_{e}} B\left(f_{e}\right) X_{c_{1}} & \text { if } \alpha>\beta \\
T^{(1+\beta) / 2} \delta_{T}^{T_{c}-T_{e}} B\left(f_{e}\right) X_{c_{2}} & \text { if } \alpha<\beta
\end{array} .\right.
$$

(4) For $T_{1} \in B$ and $T_{2} \in C$,

$$
\sum_{j=T_{1}}^{T_{2}} \tilde{X}_{j-1} \varepsilon_{j} \sim_{a}\left\{\begin{array}{ll}
T^{(1+\alpha) / 2} \delta_{T}^{T_{c}-T_{e}} B\left(f_{e}\right) X_{c_{1}} & \text { if } \alpha>\beta \\
T^{(1+\beta) / 2} \delta_{T}^{T_{c}-T_{e}} B\left(f_{e}\right) X_{c_{2}} & \text { if } \alpha<\beta
\end{array} .\right.
$$

(5) For $T_{1} \in B$ and $T_{2} \in N_{1}$,

$$
\sum_{j=T_{1}}^{T_{2}} \tilde{X}_{j-1} \varepsilon_{j} \sim_{a}\left\{\begin{array}{ll}
T^{(1+\alpha) / 2} \delta_{T}^{T_{c}-T_{e}} B\left(f_{e}\right) X_{c_{1}} & \text { if } \alpha>\beta \\
T^{(1+\beta) / 2} \delta_{T}^{T_{c}-T_{e}} B\left(f_{e}\right) X_{c_{2}} & \text { if } \alpha<\beta
\end{array} .\right.
$$

(6) For $T_{1} \in C$ and $T_{2} \in N_{1}$,

$$
\sum_{j=T_{1}}^{T_{2}} \tilde{X}_{j-1} \varepsilon_{j} \sim_{a}\left\{\begin{array}{lc}
T\left\{\frac{1}{2}\left[B\left(f_{2}\right)-B\left(f_{r}\right)\right]^{2}-\frac{1}{2}\left(f_{2}-f_{r}\right) \sigma^{2}\right. & \text { if } \alpha>\beta \\
\left.-2 \frac{f_{2}-f_{r}}{f_{w}}\left[B\left(f_{2}\right)-B\left(f_{r}\right)\right] \int_{f_{r}}^{f_{2}}\left[B(s)-B\left(f_{r}\right)\right] d s\right\} & \\
T^{(1+\beta) / 2} \gamma_{T}^{T_{1}-T_{c}-1} \delta_{T}^{T_{c}-T_{e}} B\left(f_{e}\right) X_{c_{2}} & \text { if } \alpha<\beta
\end{array} .\right.
$$

Lemma B.6 Cross-product terms involving $\tilde{X}_{j-1}$ and $\tilde{X}_{j}-\delta_{T} \tilde{X}_{j-1}$ behave as follows.

(1) For $T_{1} \in N_{0}$ and $T_{2} \in B$,

$$
\sum_{j=T_{1}}^{T_{2}} \tilde{X}_{j-1}\left(\tilde{X}_{j}-\delta_{T} \tilde{X}_{j-1}\right) \sim_{a}-T^{\alpha} \delta_{T}^{2\left(T_{2}-T_{e}\right)} \frac{f_{e}-f_{1}}{f_{w}} B\left(f_{e}\right)^{2} .
$$

(2) For $T_{1} \in N_{0}$ and $T_{2} \in C$,

$\sum_{j=T_{1}}^{T_{2}} \tilde{X}_{j-1}\left(\tilde{X}_{j}-\delta_{T} \tilde{X}_{j-1}\right) \sim_{a}\left\{\begin{array}{ll}-T^{2 \alpha-\beta} \delta_{T}^{2\left(T_{c}-T_{e}\right)} c_{2} \frac{f_{2}-f_{c}}{f_{w} c_{1}^{2}} B\left(f_{e}\right)^{2} & \text { if } \alpha>\beta \text { and } 1+\beta<2 \alpha \\ -T \delta_{T}^{2\left(T_{c}-T_{e}\right)} \frac{1}{2} B\left(f_{e}\right)^{2} & \text { if } \alpha>\beta \text { and } 1+\beta>2 \alpha \\ -T^{1+\beta-\alpha} \delta_{T}^{2\left(T_{c}-T_{e}\right)} \frac{c_{1}}{2 c_{2}} B\left(f_{e}\right)^{2} & \text { if } \alpha<\beta\end{array}\right.$.

(3) For $T_{1} \in N_{0}$ and $T_{2} \in N_{1}$,

$$
\sum_{j=T_{1}}^{T_{2}} \tilde{X}_{j-1}\left(\tilde{X}_{j}-\delta_{T} \tilde{X}_{j-1}\right) \sim_{a}\left\{\begin{array}{ll}
-T^{2 \alpha-\beta} \delta_{T}^{2\left(T_{c}-T_{e}\right)} c_{2} \frac{f_{r}-f_{c}}{f_{w} c_{1}^{2}} B\left(f_{e}\right)^{2} & \text { if } \alpha>\beta \text { and } 1+\beta<2 \alpha \\
-T \delta_{T}^{2\left(T_{c}-T_{e}\right)} \frac{1}{2} B\left(f_{e}\right)^{2} & \text { if } \alpha>\beta \text { and } 1+\beta>2 \alpha \\
-T^{1+\beta-\alpha} \delta_{T}^{2\left(T_{c}-T_{e}\right)} \frac{c_{1}}{2 c_{2}} B\left(f_{e}\right)^{2} & \text { if } \alpha<\beta
\end{array} .\right.
$$


(4) For $T_{1} \in B$ and $T_{2} \in C$,

$\sum_{j=T_{1}}^{T_{2}} \tilde{X}_{j-1}\left(\tilde{X}_{j}-\delta_{T} \tilde{X}_{j-1}\right) \sim^{a}\left\{\begin{array}{ll}-T^{2 \alpha-\beta} \delta_{T}^{2\left(T_{c}-T_{e}\right)} c_{2} \frac{f_{2}-f_{c}}{f_{w} c_{1}^{2}} B\left(f_{e}\right)^{2} & \text { if } \alpha>\beta \text { and } 1+\beta<2 \alpha \\ -T \delta_{T}^{2\left(T_{c}-T_{e}\right)} \frac{1}{2} B\left(f_{e}\right)^{2} & \text { if } \alpha>\beta \text { and } 1+\beta>2 \alpha \\ -T^{1+\beta-\alpha} \delta_{T}^{2\left(T_{c}-T_{e}\right)} \frac{c_{1}}{2 c_{2}} B\left(f_{e}\right)^{2} & \text { if } \alpha<\beta\end{array}\right.$.

(5) For $T_{1} \in B$ and $T_{2} \in N_{1}$,

$\sum_{j=T_{1}}^{T_{2}} \tilde{X}_{j-1}\left(\tilde{X}_{j}-\delta_{T} \tilde{X}_{j-1}\right) \sim^{a}\left\{\begin{array}{ll}-T^{2 \alpha-\beta} \delta_{T}^{2\left(T_{c}-T_{e}\right)} c_{2} \frac{f_{r}-f_{c}}{f_{w} c_{1}^{2}} B\left(f_{e}\right)^{2} & \text { if } \alpha>\beta \text { and } 1+\beta<2 \alpha \\ -T \delta_{T}^{2\left(T_{c}-T_{e}\right)} \frac{1}{2} B\left(f_{e}\right)^{2} & \text { if } \alpha>\beta \text { and } 1+\beta>2 \alpha \\ -T^{1+\beta-\alpha} \delta_{T}^{2\left(T_{c}-T_{e}\right)} \frac{c_{1}}{2 c_{2}} B\left(f_{e}\right)^{2} & \text { if } \alpha<\beta\end{array}\right.$.

(6) For $T_{1} \in C$ and $T_{2} \in N_{1}$,

$$
\sum_{j=T_{1}}^{T_{2}} \tilde{X}_{j-1}\left(\tilde{X}_{j}-\delta_{T} \tilde{X}_{j-1}\right) \sim_{a}\left\{\begin{array}{ll}
-T^{2-\beta} c_{2} \frac{\left(f_{r}-f_{1}\right)\left(f_{2}-f_{r}\right)^{2}}{f_{w}^{2}}\left[\int_{f_{r}}^{f_{2}}\left[B(s)-B\left(f_{r}\right)\right] d s\right]^{2} & \text { if } \alpha>\beta \\
-T^{1+\beta-\alpha} \delta_{T}^{2\left(T_{c}-T_{e}\right)} \gamma_{T}^{2\left(T_{1}-T_{c}\right)} \frac{c_{1}}{2 c_{2}} B\left(f_{e}\right)^{2} & \text { if } \alpha<\beta
\end{array} .\right.
$$

Lemma B.7 The sums of cross-products of $\tilde{X}_{j-1}$ and $\tilde{X}_{j}-\gamma_{T} \tilde{X}_{j-1}$ behave as follows.

(1) For $T_{1} \in N_{0}$ and $T_{2} \in B$,

$$
\sum_{j=T_{1}}^{T_{2}} \tilde{X}_{j-1}\left(\tilde{X}_{j}-\gamma_{T} \tilde{X}_{j-1}\right) \sim_{a}\left\{\begin{array}{ll}
T^{1+\alpha-\beta} \delta_{T}^{2\left(T_{2}-T_{e}\right)} \frac{c_{2}}{2 c_{1}} B\left(f_{e}\right)^{2} & \text { if } \alpha>\beta \\
T \delta_{T}^{2\left(T_{2}-T_{e}\right)} \frac{1}{2} B\left(f_{e}\right)^{2} & \text { if } \alpha<\beta
\end{array} .\right.
$$

(2) For $T_{1} \in N_{0}$ and $T_{2} \in C$,

$\sum_{j=T_{1}}^{T_{2}} \tilde{X}_{j-1}\left(\tilde{X}_{j}-\gamma_{T} \tilde{X}_{j-1}\right) \sim_{a}\left\{\begin{array}{ll}T^{1+\alpha-\beta} \delta_{T}^{2\left(T_{c}-T_{e}\right)} \frac{c_{2}}{2 c_{1}} B\left(f_{e}\right)^{2} & \text { if } \alpha>\beta \\ T \delta_{T}^{2\left(T_{c}-T_{e}\right)} \frac{1}{2} B\left(f_{e}\right)^{2} & \text { if } \alpha<\beta \text { and } 1+\alpha>2 \beta \\ T^{2 \beta-\alpha} \delta_{T}^{2\left(T_{c}-T_{e}\right)} c_{1} \frac{f_{c}-f_{e}}{f_{w} c_{2}^{2}} B\left(f_{e}\right)^{2} & \text { if } \alpha<\beta \text { and } 1+\alpha<2 \beta\end{array}\right.$.

(3) For $T_{1} \in N_{0}$ and $T_{2} \in N_{1}$,

$$
\sum_{j=T_{1}}^{T_{2}} \tilde{X}_{j-1}\left(\tilde{X}_{j}-\gamma_{T} \tilde{X}_{j-1}\right) \sim_{a}\left\{\begin{array}{ll}
T^{1+\alpha-\beta} \delta_{T}^{2\left(T_{c}-T_{e}\right)} \frac{c_{2}}{2 c_{1}} B\left(f_{e}\right)^{2} & \text { if } \alpha>\beta \\
T \delta_{T}^{2\left(T_{c}-T_{e}\right)} \frac{1}{2} B\left(f_{e}\right)^{2} & \text { if } \alpha<\beta \text { and } 1+\alpha>2 \beta \\
T^{2 \beta-\alpha} \delta_{T}^{2\left(T_{c}-T_{e}\right)} c_{1} \frac{f_{c}-f_{e}}{f_{w} c_{2}^{2}} B\left(f_{e}\right)^{2} & \text { if } \alpha<\beta \text { and } 1+\alpha<2 \beta
\end{array} .\right.
$$

(4) For $T_{1} \in B$ and $T_{2} \in C$,

$$
\sum_{j=T_{1}}^{T_{2}} \tilde{X}_{j-1}\left(\tilde{X}_{j}-\gamma_{T} \tilde{X}_{j-1}\right) \sim^{a}\left\{\begin{array}{ll}
T^{1+\alpha-\beta} \delta_{T}^{2\left(T_{c}-T_{e}\right)} \frac{c_{2}}{2 c_{1}} B\left(f_{e}\right)^{2} & \text { if } \alpha>\beta \\
T \delta_{T}^{2\left(T_{c}-T_{e}\right)} \frac{1}{2} B\left(f_{e}\right)^{2} & \text { if } \alpha<\beta \text { and } 1+\alpha>2 \beta \\
T^{2 \beta-\alpha} \delta_{T}^{2\left(T_{c}-T_{e}\right)} c_{1} \frac{f_{c}-f_{1}}{f_{w} c_{2}^{2}} B\left(f_{e}\right)^{2} & \text { if } \alpha<\beta \text { and } 1+\alpha<2 \beta
\end{array} .\right.
$$


(5) For $T_{1} \in B$ and $T_{2} \in N_{1}$,

$$
\sum_{j=T_{1}}^{T_{2}} \tilde{X}_{j-1}\left(\tilde{X}_{j}-\delta_{T} \tilde{X}_{j-1}\right) \sim^{a}\left\{\begin{array}{ll}
T^{1+\alpha-\beta} \delta_{T}^{2\left(T_{c}-T_{e}\right)} \frac{c_{2}}{2 c_{1}} B\left(f_{e}\right)^{2} & \text { if } \alpha>\beta \\
T \delta_{T}^{2\left(T_{c}-T_{e}\right)} \frac{1}{2} B\left(f_{e}\right)^{2} & \text { if } \alpha<\beta \text { and } 1+\alpha>2 \beta \\
T^{2 \beta-\alpha} \delta_{T}^{2\left(T_{c}-T_{e}\right)} c_{1} \frac{f_{c}-f_{1}}{f_{w} c_{2}^{2}} B\left(f_{e}\right)^{2} & \text { if } \alpha<\beta \text { and } 1+\alpha<2 \beta
\end{array} .\right.
$$

(6) For $T_{1} \in C$ and $T_{2} \in N_{1}$,

$$
\begin{aligned}
& \sum_{j=T_{1}}^{T_{2}} \tilde{X}_{j-1}\left(\tilde{X}_{j}-\gamma_{T} \tilde{X}_{j-1}\right) \\
\sim & \left\{\begin{array}{ll}
T^{2-\beta} c_{2}\left(f_{2}-f_{r}\right)\left\{\int_{f_{r}}^{f_{2}}\left[B(s)-B\left(f_{r}\right)\right]^{2} d s+\frac{\left(f_{2}-f_{r}\right)\left(f_{2}-f_{r}-2 f_{w}\right)}{f_{w}^{2}}\left[\int_{f_{r}}^{f_{2}}\left[B(s)-B\left(f_{r}\right)\right] d s\right]^{2}\right\} & \text { if } \alpha>\beta \\
T^{\beta} \delta_{T}^{2\left(T_{c}-T_{e}\right)} \gamma_{T}^{2\left(T_{1}-T_{c}\right)} \frac{f_{2}-f_{r}}{f_{w} c_{2}} B\left(f_{e}\right)^{2} & \text { if } \alpha<\beta
\end{array} .\right.
\end{aligned}
$$

Lemma B.8 The sums of cross-products of $\tilde{X}_{j-1}$ and $\tilde{X}_{j}-\tilde{X}_{j-1}$ behave as follows.

(1) For $T_{1} \in N_{0}$ and $T_{2} \in B$,

$$
\sum_{j=T_{1}}^{T_{2}} \tilde{X}_{j-1}\left(\tilde{X}_{j}-\tilde{X}_{j-1}\right) \sim_{a} T \delta_{T}^{2\left(T_{2}-T_{e}\right)} \frac{1}{2} B\left(f_{e}\right)^{2} .
$$

(2) For $T_{1} \in N_{0}$ and $T_{2} \in C$,

$$
\sum_{j=T_{1}}^{T_{2}} \tilde{X}_{j-1}\left(\tilde{X}_{j}-\tilde{X}_{j-1}\right) \sim_{a}\left\{\begin{array}{ll}
-T^{2 \alpha-\beta} \delta_{T}^{2\left(T_{c}-T_{e}\right)} c_{2} \frac{f_{2}-f_{c}}{f_{w} c_{1}^{2}} B\left(f_{e}\right)^{2} & \text { if } \alpha>\beta \text { and } 1+\beta<2 \alpha \\
o_{p}\left(T \delta_{T}^{2\left(T_{c}-T_{e}\right)}\right) & \text { if } \alpha>\beta \text { and } 1+\beta>2 \alpha \\
o_{p}\left(T \delta_{T}^{2\left(T_{c}-T_{e}\right)}\right) & \text { if } \alpha<\beta \text { and } 1+\alpha>2 \beta \\
T^{2 \beta-\alpha} \delta_{T}^{2\left(T_{c}-T_{e}\right)} c_{1} \frac{f_{e}-f_{1}}{f_{w} c_{2}^{2}} B\left(f_{e}\right)^{2} & \text { if } \alpha<\beta \text { and } 1+\alpha<2 \beta
\end{array} .\right.
$$

(3) For $T_{1} \in N_{0}$ and $T_{2} \in N_{1}$,

$$
\sum_{j=T_{1}}^{T_{2}} \tilde{X}_{j-1}\left(\tilde{X}_{j}-\tilde{X}_{j-1}\right) \sim_{a}\left\{\begin{array}{ll}
-T^{2 \alpha-\beta} \delta_{T}^{2\left(T_{c}-T_{e}\right)} c_{2} \frac{f_{2}-f_{c}}{f_{w} c_{1}^{2}} B\left(f_{e}\right)^{2} & \text { if } \alpha>\beta \text { and } 1+\beta<2 \alpha \\
o_{p}\left(T \delta_{T}^{2\left(T_{c}-T_{e}\right)}\right) & \text { if } \alpha>\beta \text { and } 1+\beta>2 \alpha \\
o_{p}\left(T \delta_{T}^{2\left(T_{c}-T_{e}\right)}\right) & \text { if } \alpha<\beta \text { and } 1+\alpha>2 \beta \\
T^{2 \beta-\alpha} \delta_{T}^{2\left(T_{c}-T_{e}\right)} c_{1} \frac{f_{c}-f_{e}}{f_{w} c_{2}^{2}} B\left(f_{e}\right)^{2} & \text { if } \alpha<\beta \text { and } 1+\alpha<2 \beta
\end{array} .\right.
$$

(4) For $T_{1} \in B$ and $T_{2} \in C$,

$$
\sum_{j=T_{1}}^{T_{2}} \tilde{X}_{j-1}\left(\tilde{X}_{j}-\tilde{X}_{j-1}\right) \sim^{a}\left\{\begin{array}{ll}
-T^{2 \alpha-\beta} \delta_{T}^{2\left(T_{c}-T_{e}\right)} c_{2} \frac{f_{2}-f_{c}}{f_{w} c_{1}^{2}} B\left(f_{e}\right)^{2} & \text { if } \alpha>\beta \text { and } 1+\beta<2 \alpha \\
o_{p}\left(T \delta_{T}^{2\left(T_{c}-T_{e}\right)}\right) & \text { if } \alpha>\beta \text { and } 1+\beta>2 \alpha \\
o_{p}\left(T \delta_{T}^{2\left(T_{c}-T_{e}\right)}\right) & \text { if } \alpha<\beta \text { and } 1+\alpha>2 \beta \\
T^{2 \beta-\alpha} \delta_{T}^{2\left(T_{c}-T_{e}\right)} c_{1} \frac{f_{c}-f_{1}}{f_{w} c_{2}^{2}} B\left(f_{e}\right)^{2} & \text { if } \alpha<\beta \text { and } 1+\alpha<2 \beta
\end{array} .\right.
$$


(5) For $T_{1} \in B$ and $T_{2} \in N_{1}$,

$$
\sum_{j=T_{1}}^{T_{2}} \tilde{X}_{j-1}\left(\tilde{X}_{j}-\tilde{X}_{j-1}\right) \sim^{a}\left\{\begin{array}{ll}
-T^{2 \alpha-\beta} \delta_{T}^{2\left(T_{c}-T_{e}\right)} c_{2} \frac{f_{2}-f_{c}}{f_{w} c_{1}^{2}} B\left(f_{e}\right)^{2} & \text { if } \alpha>\beta \text { and } 1+\beta<2 \alpha \\
o_{p}\left(T \delta_{T}^{2\left(T_{c}-T_{e}\right)}\right) & \text { if } \alpha>\beta \text { and } 1+\beta>2 \alpha \\
o_{p}\left(T \delta_{T}^{2\left(T_{c}-T_{e}\right)}\right) & \text { if } \alpha<\beta \text { and } 1+\alpha>2 \beta \\
T^{2 \beta-\alpha} \delta_{T}^{2\left(T_{c}-T_{e}\right)} c_{1} \frac{f_{c}-f_{1}}{f_{w} c_{2}^{2}} B\left(f_{e}\right)^{2} & \text { if } \alpha<\beta \text { and } 1+\alpha<2 \beta
\end{array} .\right.
$$

(6) For $T_{1} \in C$ and $T_{2} \in N_{1}$,

$$
\sum_{j=T_{1}}^{T_{2}} \tilde{X}_{j-1}\left(\tilde{X}_{j}-\tilde{X}_{j-1}\right) \sim_{a}\left\{\begin{array}{ll}
-T^{2-\beta} c_{2} \frac{\left(f_{r}-f_{1}\right)\left(f_{2}-f_{r}\right)^{2}}{f_{w}^{2}}\left[\int_{f_{r}}^{f_{2}}\left[B(s)-B\left(f_{r}\right)\right] d s\right]^{2} & \text { if } \alpha>\beta \\
-T \delta_{T}^{2\left(T_{c}-T_{e}\right)} \gamma_{T}^{2\left(T_{1}-T_{c}\right)} \frac{1}{2} B\left(f_{e}\right)^{2} & \text { if } \alpha<\beta
\end{array} .\right.
$$

\section{B.2.1 Test asymptotics}

The fitted regression model for the recursive unit root tests is

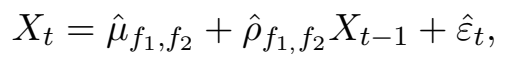

where the intercept $\hat{\mu}_{f_{1}, f_{2}}$ and slope coefficient $\hat{\rho}_{f_{1}, f_{2}}$ are obtained using data over the subperiod $\left[f_{1}, f_{2}\right]$.

Remark 1 Based on Lemma B.4 and Lemma B.6, we can obtain limit forms of $\hat{\rho}_{f_{1}, f_{2}}-\delta_{T}$ using

$$
\hat{\rho}_{f_{1}, f_{2}}-\delta_{T}=\frac{\sum_{j=T_{1}}^{T_{2}} \tilde{X}_{j-1}\left(\tilde{X}_{j}-\delta_{T} \tilde{X}_{j-1}\right)}{\sum_{j=T_{1}}^{T_{2}} \tilde{X}_{j-1}^{2}} .
$$

When $T_{1} \in N_{0}$ and $T_{2} \in B$

$$
\hat{\rho}_{f_{1}, f_{2}}-\delta_{T} \sim_{a}-\frac{1}{T} 2 c_{1} \frac{f_{e}-f_{1}}{f_{w}} .
$$

When $T_{1} \in C$ and $T_{2} \in N_{1}$,

$$
\hat{\rho}_{f_{1}, f_{2}}-\delta_{T} \sim a\left\{\begin{array}{ll}
-T^{-\beta} \frac{c_{2} \frac{\left(f_{r}-f_{1}\right)\left(f_{2}-f_{r}\right)}{f_{w}^{2}}\left[\int_{f_{r}}^{f_{2}}\left[B(s)-B\left(f_{r}\right)\right] d s\right]^{2}}{\left\{\int_{f_{r}}^{f_{2}}\left[B(s)-B\left(f_{r}\right)\right]^{2} d s-\frac{f_{2}-f_{r}}{f_{w}}\left[\int_{f_{r}}^{f_{2}}\left[B(s)-B\left(f_{r}\right)\right] d s\right]^{2}\right\}} & \text { if } \alpha>\beta \\
-T^{-\alpha} c_{1} & \text { if } \alpha<\beta
\end{array} .\right.
$$

and for all other cases

$$
\hat{\rho}_{f_{1}, f_{2}}-\delta_{T} \sim_{a} \begin{cases}-T^{\alpha-\beta-1} 2 c_{2} \frac{f_{2}-f_{c}}{f_{w} c_{1}} & \text { if } \alpha>\beta \text { and } 1+\beta<2 \alpha \\ -T^{-\alpha} c_{1} & \text { if } \alpha>\beta \text { and } 1+\beta>2 \alpha \\ -T^{-\alpha} c_{1} & \text { if } \alpha<\beta\end{cases}
$$


Remark 2 Based on Lemma B.4 and Lemma B. 7, we can obtain limit forms of $\hat{\rho}_{f_{1}, f_{2}}-\gamma_{T}$ using

$$
\hat{\rho}_{f_{1}, f_{2}}-\gamma_{T}=\frac{\sum_{j=T_{1}}^{T_{2}} \tilde{X}_{j-1}\left(\tilde{X}_{j}-\gamma_{T} \tilde{X}_{j-1}\right)}{\sum_{j=T_{1}}^{T_{2}} \tilde{X}_{j-1}^{2}} .
$$

When $T_{1} \in N_{0}$ and $T_{2} \in B$

$$
\hat{\rho}_{f_{1}, f_{2}}-\gamma_{T} \sim_{a}\left\{\begin{array}{ll}
T^{-\beta} c_{2} & \text { if } \alpha>\beta \\
T^{-\alpha} c_{1} & \text { if } \alpha<\beta
\end{array} .\right.
$$

When $T_{1} \in C$ and $T_{2} \in N_{1}$,

$$
\hat{\rho}_{f_{1}, f_{2}}-\gamma_{T} \sim_{a}\left\{\begin{array}{ll}
T^{-\beta} c_{2} \frac{\left\{\int_{f_{r}}^{f_{2}}\left[B(s)-B\left(f_{r}\right)\right]^{2} d s+\frac{\left(f_{2}-f_{r}\right)\left(f_{2}-f_{r}-2 f_{w}\right)}{f_{w}^{2}}\left[\int_{f_{r}}^{f_{2}}\left[B(s)-B\left(f_{r}\right)\right] d s\right]^{2}\right\}}{\int_{f_{r}}^{f_{2}}\left[B(s)-B\left(f_{r}\right)\right]^{2} d s-\frac{f_{2}-f_{r}}{f_{w}}\left[\int_{f_{r}}^{f_{2}}\left[B(s)-B\left(f_{r}\right)\right] d s\right]^{2}} & \text { if } \alpha>\beta \\
2 \frac{1}{T} \frac{f_{2}-f_{r}}{f_{w}} & \text { if } \alpha<\beta
\end{array} .\right.
$$

and for all other cases

$$
\hat{\rho}_{f_{1}, f_{2}}-\gamma_{T} \sim_{a} \begin{cases}T^{-\beta} c_{2} & \text { if } \alpha>\beta \\ T^{-\beta} c_{2} & \text { if } \alpha<\beta \text { and } 1+\alpha>2 \beta \\ T^{\beta-\alpha-1} 2 c_{1} \frac{f_{c}-f_{e}}{f_{w} c_{2}} & \text { if } \alpha<\beta \text { and } 1+\alpha<2 \beta\end{cases}
$$

Remark 3 Based on Lemma B.4 and Lemma B.8, we can obtain limit forms of $\hat{\rho}_{f_{1}, f_{2}}-1$ using

$$
\hat{\rho}_{f_{1}, f_{2}}-1=\frac{\sum_{j=T_{1}}^{T_{2}} \tilde{X}_{j-1}\left(\tilde{X}_{j}-\tilde{X}_{j-1}\right)}{\sum_{j=T_{1}}^{T_{2}} \tilde{X}_{j-1}^{2}}
$$

When $T_{1} \in N_{0}$ and $T_{2} \in B$

$$
\hat{\rho}_{f_{1}, f_{2}}-1 \sim_{a} \frac{c_{1}}{T^{\alpha}} .
$$

When $T_{1} \in C$ and $T_{2} \in N_{1}$,

$$
\hat{\rho}_{f_{1}, f_{2}}-1 \sim_{a}\left\{\begin{array}{ll}
-T^{-\beta} c_{2} \frac{\frac{\left(f_{r}-f_{1}\right)\left(f_{2}-f_{r}\right)}{f_{w}^{2}}\left[\int_{f_{r}}^{f_{2}}\left[B(s)-B\left(f_{r}\right)\right] d s\right]^{2}}{\left\{\int_{f_{r}}^{f_{2}}\left[B(s)-B\left(f_{r}\right)\right]^{2} d s-\frac{f_{2}-f_{r}}{f_{w}}\left[\int_{f_{r}}^{f_{2}}\left[B(s)-B\left(f_{r}\right)\right] d s\right]^{2}\right\}} & \text { if } \alpha>\beta \\
-T^{-\beta} c_{2} & \text { if } \alpha<\beta
\end{array} .\right.
$$

And for all other cases

$$
\hat{\rho}_{f_{1}, f_{2}}-1 \sim_{a} \begin{cases}-T^{\alpha-\beta-1} 2 c_{2} \frac{f_{2}-f_{c}}{f_{w} c_{1}} & \text { if } \alpha>\beta \text { and } 1+\beta<2 \alpha \\ o_{p}\left(T^{-\alpha}\right) & \text { if } \alpha>\beta \text { and } 1+\beta>2 \alpha \\ o_{p}\left(T^{-\beta}\right) & \text { if } \alpha<\beta \text { and } 1+\alpha>2 \beta \\ T^{\beta-\alpha-1} 2 c_{1} \frac{f_{e}-f_{1}}{f_{w} c_{2}} & \text { if } \alpha<\beta \text { and } 1+\alpha<2 \beta\end{cases}
$$


Based on the above three remarks, one can see that the quantity $\hat{\rho}_{f_{1}, f_{2}}-\delta_{T}$ diverges to negative infinity and the quantity $\hat{\rho}_{f_{1}, f_{2}}-\gamma_{T}$ diverges to positive infinity. In other words, the estimated value of $\hat{\rho}_{f_{1}, f_{2}}$ is bounded by $\delta_{T}$ and $\gamma_{T}$. Furthermore, the quantity $\hat{\rho}_{f_{1}, f_{2}}-1$ diverges to positive infinity when $T_{1} \in N_{0}$ and $T_{2} \in B$ and negative infinity when $T_{1} \in C$ and $T_{2} \in N_{1}$.

Lemma B.9 To obtain the asymptotic behaviors of the Dickey-Fuller t-statistic, we first obtain the equation standard error of the regression over $\left[T_{1}, T_{2}\right]$ which is

$$
\hat{\sigma}_{f_{1} f_{2}}=\left\{T_{w}^{-1} \sum_{j=T_{1}}^{T_{2}}\left(\tilde{X}_{j}-\hat{\rho}_{f_{1}, f_{2}} \tilde{X}_{j-1}\right)^{2}\right\}^{1 / 2} .
$$

(1) When $T_{1} \in N_{0}$ and $T_{2} \in B$,

$$
\hat{\sigma}_{f_{1} f_{2}}^{2}=O_{p}\left(T^{-1} \delta_{T}^{2\left(T_{2}-T_{e}\right)}\right)
$$

(2) When $T_{1} \in N_{0}$ and $T_{2} \in C$,

$$
\hat{\sigma}_{f_{1} f_{2}}^{2}= \begin{cases}O_{p}\left(T^{2 \alpha-2 \beta-1} \delta_{T}^{2\left(T_{c}-T_{e}\right)}\right) & \text { if } \alpha>\beta \text { and } 1+\beta<2 \alpha \\ O_{p}\left(T^{-\beta} \delta_{T}^{2\left(T_{c}-T_{e}\right)}\right) & \text { if } \alpha>\beta \text { and } 1+\beta>2 \alpha \\ O_{p}\left(T^{-\alpha} \delta_{T}^{2\left(T_{c}-T_{e}\right)}\right) & \text { if } \alpha<\beta \text { and } 1+\alpha>2 \beta \\ O_{p}\left(T^{2 \beta-2 \alpha-1} \delta_{T}^{2\left(T_{c}-T_{e}\right)}\right) & \text { if } \alpha<\beta \text { and } 1+\alpha<2 \beta\end{cases}
$$

(3) When $T_{1} \in N_{0}$ and $T_{2} \in N_{1}$,

$$
\hat{\sigma}_{f_{1} f_{2}}^{2} \sim_{a}\left\{\begin{array}{ll}
O_{p}\left(T^{2 \alpha-2 \beta-1} \delta_{T}^{2\left(T_{c}-T_{e}\right)}\right) & \text { if } \alpha>\beta \text { and } 1+\beta<2 \alpha \\
O_{p}\left(T^{-\beta} \delta_{T}^{2\left(T_{c}-T_{e}\right)}\right) & \text { if } \alpha>\beta \text { and } 1+\beta>2 \alpha \\
O_{p}\left(T^{-\alpha} \delta_{T}^{2\left(T_{c}-T_{e}\right)}\right) & \text { if } \alpha<\beta \text { and } 1+\alpha>2 \beta \\
O_{p}\left(T^{2 \beta-2 \alpha-1} \delta_{T}^{2\left(T_{c}-T_{e}\right)}\right) & \text { if } \alpha<\beta \text { and } 1+\alpha<2 \beta
\end{array} .\right.
$$

(4) When $T_{1} \in B$ and $T_{2} \in C$,

$$
\hat{\sigma}_{f_{1} f_{2}}^{2} \sim_{a}\left\{\begin{array}{ll}
O_{p}\left(T^{2 \alpha-2 \beta-1} \delta_{T}^{2\left(T_{c}-T_{e}\right)}\right) & \text { if } \alpha>\beta \text { and } 1+\beta<2 \alpha \\
O_{p}\left(T^{-\beta} \delta_{T}^{2\left(T_{c}-T_{e}\right)}\right) & \text { if } \alpha>\beta \text { and } 1+\beta>2 \alpha \\
O_{p}\left(T^{-\alpha} \delta_{T}^{2\left(T_{c}-T_{e}\right)}\right) & \text { if } \alpha<\beta \text { and } 1+\alpha>2 \beta \\
O_{p}\left(T^{2 \beta-2 \alpha-1} \delta_{T}^{2\left(T_{c}-T_{e}\right)}\right) & \text { if } \alpha<\beta \text { and } 1+\alpha<2 \beta
\end{array} .\right.
$$


(5) When $T_{1} \in B$ and $T_{2} \in N_{1}$,

$$
\hat{\sigma}_{f_{1} f_{2}}^{2} \sim_{a} \begin{cases}O_{p}\left(T^{2 \alpha-2 \beta-1} \delta_{T}^{2\left(T_{c}-T_{e}\right)}\right) & \text { if } \alpha>\beta \text { and } 1+\beta<2 \alpha \\ O_{p}\left(T^{-\beta} \delta_{T}^{2\left(T_{c}-T_{e}\right)}\right) & \text { if } \alpha>\beta \text { and } 1+\beta>2 \alpha \\ O_{p}\left(T^{-\alpha} \delta_{T}^{2\left(T_{c}-T_{e}\right)}\right) & \text { if } \alpha<\beta \text { and } 1+\alpha>2 \beta \\ O_{p}\left(T^{2 \beta-2 \alpha-1} \delta_{T}^{2\left(T_{c}-T_{e}\right)}\right) & \text { if } \alpha<\beta \text { and } 1+\alpha<2 \beta\end{cases}
$$

(6) When $T_{1} \in C$ and $T_{2} \in N_{1}$,

$$
\hat{\sigma}_{f_{1} f_{2}}^{2} \sim_{a}\left\{\begin{array}{ll}
O_{p}\left(T^{1-2 \beta}\right) & \text { if } \alpha>\beta \\
O_{p}\left(T^{\beta-2 \alpha} \delta_{T}^{2\left(T_{c}-T_{e}\right)} \gamma_{T}^{2\left(T_{1}-T_{c}\right)}\right) & \text { if } \alpha<\beta
\end{array} .\right.
$$

The asymptotic distribution of the Dickey-Fuller t statistic can be calculated as follows

$$
D F_{f_{1}, f_{2}}^{t}=\left(\frac{\sum_{j=T_{1}}^{T_{2}} \tilde{X}_{j-1}^{2}}{\hat{\sigma}_{f_{1} f_{2}}^{2}}\right)^{1 / 2}\left(\hat{\rho}_{f_{1}, f_{2}}-1\right)
$$

Notice that the sign of the DF statistics depend on that of $\hat{\rho}_{f_{1}, f_{2}}-1$.

Remark 4 (1) When $T_{1} \in N_{0}$ and $T_{2} \in B$

$$
D F_{f_{1}, f_{2}}^{t}=\left(\frac{\sum_{j=T_{1}}^{T_{2}} \tilde{X}_{j-1}^{2}}{\hat{\sigma}_{f_{1} f_{2}}^{2}}\right)^{1 / 2}\left(\hat{\rho}_{f_{1}, f_{2}}-1\right)=O_{p}\left(T^{1-\alpha / 2}\right) \rightarrow+\infty .
$$

When $T_{1} \in C$ and $T_{2} \in N_{1}$

$$
D F_{f_{1}, f_{2}}^{t}=\left(\frac{\sum_{j=T_{1}}^{T_{2}} \tilde{X}_{j-1}^{2}}{\hat{\sigma}_{f_{1} f_{2}}^{2}}\right)^{1 / 2}\left(\hat{\rho}_{f_{1}, f_{2}}-1\right)=\left\{\begin{array}{ll}
O_{p}\left(T^{1 / 2}\right) \rightarrow-\infty & \text { if } \alpha>\beta \\
O_{p}\left(T^{1 / 2+\alpha-\beta}\right) \rightarrow-\infty & \text { if } \alpha<\beta
\end{array} .\right.
$$

For all other cases

$$
D F_{f_{1}, f_{2}}^{t}=\left(\frac{\sum_{j=T_{1}}^{T_{2}} \tilde{X}_{j-1}^{2}}{\hat{\sigma}_{f_{1} f_{2}}^{2}}\right)^{1 / 2}\left(\hat{\rho}_{f_{1}, f_{2}}-1\right)=\left\{\begin{array}{ll}
O_{p}\left(T^{\alpha / 2}\right) \rightarrow-\infty & \text { if } \alpha>\beta \text { and } 1+\beta<2 \alpha \\
o_{p}\left(T^{(1-\alpha+\beta) / 2}\right) & \text { if } \alpha>\beta \text { and } 1+\beta>2 \alpha \\
o_{p}\left(T^{(1-\beta+\alpha) / 2}\right) & \text { if } \alpha<\beta \text { and } 1+\alpha>2 \beta \\
O_{p}\left(T^{\beta / 2}\right) \rightarrow+\infty & \text { if } \alpha<\beta \text { and } 1+\alpha<2 \beta
\end{array} .\right.
$$

Given that $f_{2}=f$ and $f_{1} \in\left[0, f-f_{0}\right]$, the asymptotic behavior of the backward sup DF statistic under the alternative hypothesis are:

$$
B S D F_{f}\left(f_{0}\right) \sim \begin{cases}F_{f}\left(W, f_{0}\right) \\ O_{p}\left(T^{1-\alpha / 2}\right) \rightarrow+\infty & \text { if } f \in N_{0}\end{cases}
$$


This proves Theorem 3.2. Following the standard probability arguments (see PSY), we deduce that $\operatorname{Pr}\left\{\left|\hat{f}_{e}-f_{e}\right|>\eta\right\} \rightarrow 0$ and $\operatorname{Pr}\left\{\left|\hat{f}_{c}-f_{c}\right|>\gamma\right\} \rightarrow 0$ for any $\eta, \gamma>0$ as $T \rightarrow \infty$, provided that

$$
\left\{\begin{array}{ll}
\frac{T^{\alpha / 2}}{s c v^{\beta} T}+\frac{s c v^{\beta} T}{T^{1-\alpha / 2}} \rightarrow 0 & \text { if } \alpha>\beta \text { and } 1+\beta<2 \alpha \\
\frac{T^{(1-\alpha+\beta} / 2}{s c v^{\beta} T}+\frac{s c v^{\beta} T}{T^{1-\alpha / 2}} \rightarrow 0 & \text { if } \alpha>\beta \text { and } 1+\beta>2 \alpha \\
\frac{T^{(1-\beta+\alpha) / 2}}{s c v^{\beta} T}+\frac{s c v^{\beta}}{T^{1-\alpha / 2}} \rightarrow 0 & \text { if } \alpha<\beta \text { and } 1+\alpha>2 \beta \\
\frac{T^{\beta / 2}}{s c v^{\beta} T}+\frac{s c v^{\beta} T}{T^{1-\alpha / 2}} \rightarrow 0 & \text { if } \alpha<\beta \text { and } 1+\alpha<2 \beta
\end{array} .\right.
$$

Therefore, $\hat{f}_{e}$ and $\hat{f}_{c}$ are consistent estimators of $f_{e}$ and $f_{c}$. This proves Theorem 3.3.

\section{B.3 Dating Bubble Contractions}

Define the demeaned quantity as $\tilde{X}_{t}^{*} \equiv X_{t}^{*}-\frac{1}{\tau_{w}} \sum_{j=\tau_{1}}^{\tau_{2}} X_{j}^{*}$. Since $\tau_{w}=T_{w}$ and $\sum_{j=\tau_{1}}^{\tau_{2}} X_{j}^{*}=$ $\sum_{i=T_{1}}^{T_{2}} X_{i}$, we have

$$
\tilde{X}_{t}^{*}=X_{t}^{*}-\frac{1}{T_{w}} \sum_{j=T_{1}}^{T_{2}} X_{j}^{*}=X_{T+1-t}-\frac{1}{T_{w}} \sum_{i=T_{1}}^{T_{2}} X_{i}=\tilde{X}_{T+1-t} .
$$

Based on this linkage, we derive the next two lemmas.

Lemma B.10 Quadratic terms in $\tilde{X}_{t}^{*}$ behave as follows.

(1) For $\tau_{1} \in B$ and $\tau_{2} \in N_{0}$,

$$
\sum_{j=\tau_{1}}^{\tau_{2}} \tilde{X}_{j-1}^{* 2}=\sum_{j=T_{1}}^{T_{2}} \tilde{X}_{j+1}^{2} \sim_{a} T^{1+\alpha} \delta_{T}^{2\left(T_{2}-T_{e}\right)} \frac{1}{2 c_{1}} B\left(f_{e}\right)^{2}
$$

(2) For $\tau_{1} \in C$ and $\tau_{2} \in N_{0}$,

$$
\sum_{j=\tau_{1}}^{\tau_{2}} \tilde{X}_{j-1}^{* 2}=\sum_{j=T_{1}}^{T_{2}} \tilde{X}_{j+1}^{2} \sim_{a}\left\{\begin{array}{ll}
T^{1+\alpha} \delta_{T}^{2\left(T_{c}-T_{e}\right)} \frac{1}{2 c_{1}} B\left(f_{e}\right)^{2} & \text { if } \alpha>\beta \\
T^{1+\beta} \delta_{T}^{2\left(T_{c}-T_{e}\right)} \frac{1}{2 c_{2}} B\left(f_{e}\right)^{2} & \text { if } \alpha<\beta
\end{array} .\right.
$$

(3) For $\tau_{1} \in N_{1}$ and $\tau_{2} \in N_{0}$,

$$
\sum_{j=\tau_{1}}^{\tau_{2}} \tilde{X}_{j-1}^{* 2}=\sum_{j=T_{1}}^{T_{2}} \tilde{X}_{j+1}^{2} \sim_{a}\left\{\begin{array}{ll}
T^{1+\alpha} \delta_{T}^{2\left(T_{c}-T_{e}\right)} \frac{1}{2 c_{1}} B\left(f_{e}\right)^{2} & \text { if } \alpha>\beta \\
T^{1+\beta} \delta_{T}^{2\left(T_{c}-T_{e}\right)} \frac{1}{2 c_{2}} B\left(f_{e}\right)^{2} & \text { if } \alpha \leq \beta
\end{array} .\right.
$$

(4) For $\tau_{1} \in C$ and $\tau_{2} \in B$,

$$
\sum_{j=\tau_{1}}^{\tau_{2}} \tilde{X}_{j-1}^{* 2}=\sum_{j=T_{1}}^{T_{2}} \tilde{X}_{j+1}^{2} \sim_{a} \begin{cases}T^{1+\alpha} \delta_{T}^{2\left(T_{c}-T_{e}\right)} \frac{1}{2 c_{1}} B\left(f_{e}\right)^{2} & \text { if } \alpha>\beta \\ T^{1+\beta} \delta_{T}^{2\left(T_{c}-T_{e}\right)} \frac{1}{2 c_{2}} B\left(f_{e}\right)^{2} & \text { if } \alpha<\beta\end{cases}
$$


(5) For $\tau_{1} \in N_{1}$ and $\tau_{2} \in B$,

$$
\sum_{j=\tau_{1}}^{\tau_{2}} \tilde{X}_{j-1}^{* 2}=\sum_{j=T_{1}}^{T_{2}} \tilde{X}_{j+1}^{2} \sim\left\{\begin{array}{ll}
T^{1+\alpha} \delta_{T}^{2\left(T_{c}-T_{e}\right)} \frac{1}{2 c_{1}} B\left(f_{e}\right)^{2} & \text { if } \alpha>\beta \\
T^{1+\beta} \delta_{T}^{2\left(T_{c}-T_{e}\right)} \frac{1}{2 c_{2}} B\left(f_{e}\right)^{2} & \text { if } \alpha<\beta
\end{array} .\right.
$$

(6) For $\tau_{1} \in N_{1}$ and $\tau_{2} \in C$,

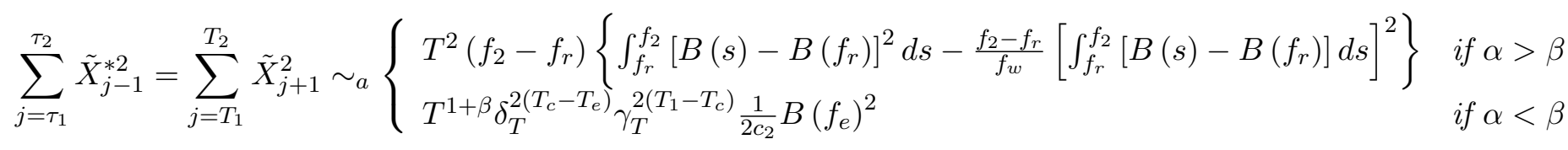

Lemma B.11 Cross-product terms involving $\tilde{X}_{t}^{*}$ and $v_{t}$ behave as follows.

(1) For $\tau_{1} \in B$ and $\tau_{2} \in N_{0}$,

$$
\sum_{j=\tau_{1}}^{\tau_{2}} \tilde{X}_{j-1}^{*} v_{j} \sim_{a}-T^{(1+\alpha) / 2} \delta_{T}^{T_{2}-T_{e}} X_{c_{1}} B\left(f_{e}\right)
$$

(2) For $\tau_{1} \in C$ and $\tau_{2} \in N_{0}$,

$$
\sum_{j=\tau_{1}}^{\tau_{2}} \tilde{X}_{j-1}^{*} v_{j} \sim_{a}\left\{\begin{array}{ll}
-T^{(1+\alpha) / 2} \delta_{T}^{T_{c}-T_{e}} B\left(f_{e}\right) X_{c_{1}} & \text { if } \alpha>\beta \\
-T^{(1+\beta) / 2} \delta_{T}^{T_{c}-T_{e}} B\left(f_{e}\right) X_{c_{2}} & \text { if } \alpha<\beta
\end{array} .\right.
$$

(3) For $\tau_{1} \in N_{1}$ and $\tau_{2} \in N_{0}$,

$$
\sum_{j=\tau_{1}}^{\tau_{2}} \tilde{X}_{j-1} v_{j} \sim_{a}\left\{\begin{array}{ll}
-T^{(1+\alpha) / 2} \delta_{T}^{T_{c}-T_{e}} B\left(f_{e}\right) X_{c_{1}} & \text { if } \alpha>\beta \\
-T^{(1+\beta) / 2} \delta_{T}^{T_{c}-T_{e}} B\left(f_{e}\right) X_{c_{2}} & \text { if } \alpha<\beta
\end{array} .\right.
$$

(4) For $\tau_{1} \in C$ and $\tau_{2} \in B$,

$$
\sum_{j=\tau_{1}}^{\tau_{2}} \tilde{X}_{j-1}^{*} v_{j} \sim_{a}\left\{\begin{array}{ll}
-T^{(1+\alpha) / 2} \delta_{T}^{T_{c}-T_{e}} B\left(f_{e}\right) X_{c_{1}} & \text { if } \alpha>\beta \\
-T^{(1+\beta) / 2} \delta_{T}^{T_{c}-T_{e}} B\left(f_{e}\right) X_{c_{2}} & \text { if } \alpha<\beta
\end{array} .\right.
$$

(5) For $\tau_{1} \in C$ and $\tau_{2} \in N_{0}$,

$$
\sum_{j=\tau_{1}}^{\tau_{2}} \tilde{X}_{j-1}^{*} v_{j} \sim_{a}\left\{\begin{array}{ll}
-T^{(1+\alpha) / 2} \delta_{T}^{T_{c}-T_{e}} B\left(f_{e}\right) X_{c_{1}} & \text { if } \alpha>\beta \\
-T^{(1+\beta) / 2} \delta_{T}^{T_{c}-T_{e}} B\left(f_{e}\right) X_{c_{2}} & \text { if } \alpha<\beta
\end{array} .\right.
$$

(6) For $\tau_{1} \in C$ and $\tau_{2} \in N_{1}$,

$$
\sum_{j=\tau_{1}}^{\tau_{2}} \tilde{X}_{j-1}^{*} v_{j} \sim_{a} \begin{cases}-T\left\{\frac{1}{2}\left[B\left(f_{2}\right)-B\left(f_{r}\right)\right]^{2}+\frac{1}{2}\left(f_{2}-f_{r}\right) \sigma^{2}\right. & \text { if } \alpha>\beta \\ \left.-\frac{f_{2}-f_{r}}{f_{w}}\left[B\left(f_{2}\right)-2 B\left(f_{r}\right)+B\left(f_{1}\right)\right] \int_{f_{r}}^{f_{2}}\left[B(s)-B\left(f_{r}\right)\right] d s\right\} & \\ -T^{(1+\beta) / 2} \gamma_{T}^{T_{1}-T_{c}} \delta_{T}^{T_{c}-T_{e}} B\left(f_{e}\right) X_{c_{2}} & \text { if } \alpha<\beta\end{cases}
$$


Lemma B.12 Cross-product terms involving $\tilde{X}_{j-1}^{*}$ and $\tilde{X}_{j}^{*}-\gamma_{T}^{-1} \tilde{X}_{j-1}^{*}$ behave as follows.

(1) For $\tau_{1} \in B$ and $\tau_{2} \in N_{0}$,

$$
\sum_{j=\tau_{1}}^{\tau_{2}} \tilde{X}_{j-1}^{*}\left(\tilde{X}_{j}^{*}-\gamma_{T}^{-1} \tilde{X}_{j-1}^{*}\right) \sim_{a}\left\{\begin{array}{ll}
-T^{1+\alpha-\beta} \delta_{T}^{2\left(T_{2}-T_{e}\right)} \frac{c_{2}}{2 c_{1}} B\left(f_{e}\right)^{2} & \text { if } \alpha>\beta \\
-T \delta_{T}^{2\left(T_{2}-T_{e}\right)} \frac{1}{2} B\left(f_{e}\right)^{2} & \text { if } \alpha<\beta
\end{array} .\right.
$$

(2) For $\tau_{1} \in C$ and $\tau_{2} \in N_{0}$,

$$
\sum_{j=\tau_{1}}^{\tau_{2}} \tilde{X}_{j-1}^{*}\left(\tilde{X}_{j}^{*}-\gamma_{T}^{-1} \tilde{X}_{j-1}^{*}\right) \sim_{a}\left\{\begin{array}{ll}
-T^{1+\alpha-\beta} \delta_{T}^{2\left(T_{c}-T_{e}\right)} \frac{c_{2}}{2 c_{1}} B\left(f_{e}\right)^{2} & \text { if } \alpha>\beta \\
-T \delta_{T}^{2\left(T_{c}-T_{e}\right)} \frac{1}{2} B\left(f_{e}\right)^{2} & \text { if } \alpha<\beta \text { and } 1+\alpha>2 \beta . \\
-T^{2 \beta-\alpha} \delta_{T}^{2\left(T_{c}-T_{e}\right)} c_{1} \frac{f_{c}-f_{e}}{f_{w} c_{2}^{2}} B\left(f_{e}\right)^{2} & \text { if } \alpha<\beta \text { and } 1+\alpha<2 \beta
\end{array} .\right.
$$

(3) For $\tau_{1} \in N_{1}$ and $\tau_{2} \in N_{0}$,

$$
\sum_{j=\tau_{1}}^{\tau_{2}} \tilde{X}_{j-1}^{*}\left(\tilde{X}_{j}^{*}-\gamma_{T}^{-1} \tilde{X}_{j-1}^{*}\right) \sim_{a}\left\{\begin{array}{ll}
-T^{1+\alpha-\beta} \delta_{T}^{2\left(T_{c}-T_{e}\right)} \frac{c_{2}}{2 c_{1}} B\left(f_{e}\right)^{2} & \text { if } \alpha>\beta \\
-T \delta_{T}^{2\left(T_{c}-T_{e}\right)} \frac{1}{2} B\left(f_{e}\right)^{2} & \text { if } \alpha<\beta \text { and } 1+\alpha>2 \beta \\
-T^{2 \beta-\alpha} \delta_{T}^{2\left(T_{c}-T_{e}\right)} c_{1} \frac{f_{c}-f_{e}}{f_{w} c_{2}^{2}} B\left(f_{e}\right)^{2} & \text { if } \alpha<\beta \text { and } 1+\alpha<2 \beta
\end{array} .\right.
$$

(4) For $\tau_{1} \in C$ and $\tau_{2} \in B$,

$$
\sum_{j=\tau_{1}}^{\tau_{2}} \tilde{X}_{j-1}^{*}\left(\tilde{X}_{j}^{*}-\gamma_{T}^{-1} \tilde{X}_{j-1}^{*}\right) \sim_{a}\left\{\begin{array}{ll}
-T^{1+\alpha-\beta} \delta_{T}^{2\left(T_{c}-T_{e}\right)} \frac{c_{2}}{2 c_{1}} B\left(f_{e}\right)^{2} & \text { if } \alpha>\beta \\
-T \delta_{T}^{2\left(T_{c}-T_{e}\right)} \frac{1}{2} B\left(f_{e}\right)^{2} & \text { if } \alpha<\beta \text { and } 1+\alpha>2 \beta \\
-T^{2 \beta-\alpha} \delta_{T}^{2\left(T_{c}-T_{e}\right)} c_{1} \frac{f_{c}-f_{1}}{f_{w} c_{2}^{2}} B\left(f_{e}\right)^{2} & \text { if } \alpha<\beta \text { and } 1+\alpha<2 \beta
\end{array} .\right.
$$

(5) For $\tau_{1} \in N_{1}$ and $\tau_{2} \in B$,

$$
\sum_{j=\tau_{1}}^{\tau_{2}} \tilde{X}_{j-1}^{*}\left(\tilde{X}_{j}^{*}-\gamma_{T}^{-1} \tilde{X}_{j-1}^{*}\right) \sim_{a}\left\{\begin{array}{ll}
-T^{1+\alpha-\beta} \delta_{T}^{2\left(T_{c}-T_{e}\right)} \frac{c_{2}}{2 c_{1}} B\left(f_{e}\right)^{2} & \text { if } \alpha>\beta \\
-T \delta_{T}^{2\left(T_{c}-T_{e}\right)} \frac{1}{2} B\left(f_{e}\right)^{2} & \text { if } \alpha<\beta \text { and } 1+\alpha>2 \beta . \\
-T^{2 \beta-\alpha} \delta_{T}^{2\left(T_{c}-T_{e}\right)} c_{1} \frac{f_{c}-f_{1}}{f_{w} c_{2}^{2}} B\left(f_{e}\right)^{2} & \text { if } \alpha<\beta \text { and } 1+\alpha<2 \beta
\end{array} .\right.
$$

(6) For $\tau_{1} \in N_{1}$ and $\tau_{2} \in C$,

$$
\begin{aligned}
& \sum_{j=\tau_{1}}^{\tau_{2}} \tilde{X}_{j-1}^{*}\left(\tilde{X}_{j}^{*}-\gamma_{T}^{-1} \tilde{X}_{j-1}^{*}\right) \\
& \sim a \begin{cases}-T^{2-\beta} c_{2}\left(f_{2}-f_{r}\right)\left\{\int_{f_{r}}^{f_{2}}\left[B(s)-B\left(f_{r}\right)\right]^{2} d s+\frac{\left(f_{2}-f_{r}\right)\left(f_{2}-f_{r}-2 f_{w}\right)}{f_{w}^{2}}\left[\int_{f_{r}}^{f_{2}}\left[B(s)-B\left(f_{r}\right)\right] d s\right]^{2}\right\} & \text { if } \alpha>\beta \\
-T^{\beta} \delta_{T}^{2\left(T_{c}-T_{e}\right)} \gamma_{T}^{2\left(T_{1}-T_{c}\right)} \frac{f_{2}-f_{r}}{f_{w} c_{2}} B\left(f_{e}\right)^{2} & \text { if } \alpha<\beta\end{cases}
\end{aligned}
$$

Lemma B.13 The sums of cross-products of $\tilde{X}_{j-1}^{*}$ and $\tilde{X}_{j}^{*}-\delta_{T}^{-1} \tilde{X}_{j-1}^{*}$ behave as follows. 
(1) For $\tau_{1} \in B$ and $\tau_{2} \in N_{0}$,

$$
\sum_{j=\tau_{1}}^{\tau_{2}} \tilde{X}_{j-1}^{*}\left(\tilde{X}_{j}^{*}-\delta_{T}^{-1} \tilde{X}_{j-1}^{*}\right) \sim_{a} T^{\alpha} \delta_{T}^{2\left(T_{2}-T_{e}\right)} \frac{f_{e}-f_{1}}{f_{w}} B\left(f_{e}\right)^{2} .
$$

(2) For $\tau_{1} \in C$ and $\tau_{2} \in N_{0}$,

$\sum_{j=\tau_{1}}^{\tau_{2}} \tilde{X}_{j-1}^{*}\left(\tilde{X}_{j}^{*}-\delta_{T}^{-1} \tilde{X}_{j-1}^{*}\right) \sim_{a}\left\{\begin{array}{ll}T^{2 \alpha-\beta} \delta_{T}^{2\left(T_{c}-T_{e}\right)} c_{2} \frac{f_{2}-f_{c}}{f_{w} c_{1}^{2}} B\left(f_{e}\right)^{2} & \text { if } \alpha>\beta \text { and } 1+\beta<2 \alpha \\ T \delta_{T}^{2\left(T_{c}-T_{e}\right)} \frac{1}{2} B\left(f_{e}\right)^{2} & \text { if } \alpha>\beta \text { and } 1+\beta>2 \alpha \\ T^{1+\beta-\alpha} \delta_{T}^{2\left(T_{c}-T_{e}\right)} \frac{c_{1}}{2 c_{2}} B\left(f_{e}\right)^{2} & \text { if } \alpha<\beta\end{array}\right.$.

(3) For $\tau_{1} \in N_{1}$ and $\tau_{2} \in N_{0}$,

$\sum_{j=\tau_{1}}^{\tau_{2}} \tilde{X}_{j-1}^{*}\left(\tilde{X}_{j}^{*}-\delta_{T}^{-1} \tilde{X}_{j-1}^{*}\right) \sim_{a}\left\{\begin{array}{ll}T^{2 \alpha-\beta} \delta_{T}^{2\left(T_{c}-T_{e}\right)} c_{2} \frac{f_{r}-f_{c}}{f_{w} c_{1}^{2}} B\left(f_{e}\right)^{2} & \text { if } \alpha>\beta \text { and } 1+\beta<2 \alpha \\ T \delta_{T}^{2\left(T_{c}-T_{e}\right)} \frac{1}{2} B\left(f_{e}\right)^{2} & \text { if } \alpha>\beta \text { and } 1+\beta>2 \alpha \\ T^{1+\beta-\alpha} \delta_{T}^{2\left(T_{c}-T_{e}\right)} \frac{c_{1}}{2 c_{2}} B\left(f_{e}\right)^{2} & \text { if } \alpha<\beta\end{array}\right.$.

(4) For $\tau_{1} \in C$ and $\tau_{2} \in B$,

$\sum_{j=\tau_{1}}^{\tau_{2}} \tilde{X}_{j-1}^{*}\left(\tilde{X}_{j}^{*}-\delta_{T}^{-1} \tilde{X}_{j-1}^{*}\right) \sim_{a}\left\{\begin{array}{ll}T^{2 \alpha-\beta} \delta_{T}^{2\left(T_{c}-T_{e}\right)} c_{2} \frac{f_{2}-f_{c}}{f_{w} c_{1}^{2}} B\left(f_{e}\right)^{2} & \text { if } \alpha>\beta \text { and } 1+\beta<2 \alpha \\ T \delta_{T}^{2\left(T_{c}-T_{e}\right)} \frac{1}{2} B\left(f_{e}\right)^{2} & \text { if } \alpha>\beta \text { and } 1+\beta>2 \alpha \\ T^{1+\beta-\alpha} \delta_{T}^{2\left(T_{c}-T_{e}\right)} \frac{c_{1}}{2 c_{2}} B\left(f_{e}\right)^{2} & \text { if } \alpha<\beta\end{array}\right.$.

(5) For $\tau_{1} \in N_{1}$ and $\tau_{2} \in B$,

$\sum_{j=\tau_{1}}^{\tau_{2}} \tilde{X}_{j-1}^{*}\left(\tilde{X}_{j}^{*}-\delta_{T}^{-1} \tilde{X}_{j-1}^{*}\right) \sim_{a}\left\{\begin{array}{ll}T^{2 \alpha-\beta} \delta_{T}^{2\left(T_{c}-T_{e}\right)} c_{2} \frac{f_{r}-f_{c}}{f_{w} c_{1}^{2}} B\left(f_{e}\right)^{2} & \text { if } \alpha>\beta \text { and } 1+\beta<2 \alpha \\ T \delta_{T}^{2\left(T_{c}-T_{e}\right)} \frac{1}{2} B\left(f_{e}\right)^{2} & \text { if } \alpha>\beta \text { and } 1+\beta>2 \alpha \\ T^{1+\beta-\alpha} \delta_{T}^{2\left(T_{c}-T_{e}\right)} \frac{c_{1}}{2 c_{2}} B\left(f_{e}\right)^{2} & \text { if } \alpha<\beta\end{array}\right.$.

(6) For $\tau_{1} \in N_{1}$ and $\tau_{2} \in C$,

$$
\begin{aligned}
& \sum_{j=\tau_{1}}^{\tau_{2}} \tilde{X}_{j-1}^{*}\left(\tilde{X}_{j}^{*}-\delta_{T}^{-1} \tilde{X}_{j-1}^{*}\right) \\
\sim & \left\{\begin{array}{ll}
T^{2-\alpha} c_{1}\left(f_{2}-f_{r}\right)\left\{\int_{f_{r}}^{f_{2}}\left[B(s)-B\left(f_{r}\right)\right]^{2} d s+\frac{\left(f_{2}-f_{r}\right)\left(f_{2}-f_{r}-2 f_{w}\right)}{f_{w}^{2}}\left[\int_{f_{r}}^{f_{2}}\left[B(s)-B\left(f_{r}\right)\right] d s\right]^{2}\right\} & \text { if } \alpha>\beta \\
T^{2 \beta-\alpha} \delta_{T}^{2\left(T_{c}-T_{e}\right)} \gamma_{T}^{2\left(T_{1}-T_{c}\right)} c_{1} \frac{f_{2}-f_{r}}{f_{w} c_{2}^{2}} B\left(f_{e}\right)^{2} & \text { if } \alpha<\beta
\end{array} .\right.
\end{aligned}
$$

Lemma B.14 The sums of cross-products of $\tilde{X}_{j-1}^{*}$ and $\tilde{X}_{j}^{*}-\tilde{X}_{j-1}^{*}$ behave as follows.

(1) For $\tau_{1} \in B$ and $\tau_{2} \in N_{0}$,

$$
\sum_{j=\tau_{1}}^{\tau_{2}} \tilde{X}_{j-1}^{*}\left(\tilde{X}_{j}^{*}-\tilde{X}_{j-1}^{*}\right) \sim_{a} T \delta_{T}^{2\left(T_{2}-T_{e}\right)} \frac{1}{2} B\left(f_{e}\right)^{2} .
$$


(2) For $\tau_{1} \in C$ and $\tau_{2} \in N_{0}$,

$$
\sum_{j=\tau_{1}}^{\tau_{2}} \tilde{X}_{j-1}^{*}\left(\tilde{X}_{j}^{*}-\tilde{X}_{j-1}^{*}\right) \sim_{a}\left\{\begin{array}{cl}
T^{2 \alpha-\beta} \delta_{T}^{2\left(T_{c}-T_{e}\right)} c_{2} \frac{f_{2}-f_{c}}{f_{w} c_{1}^{2}} B\left(f_{e}\right)^{2} & \text { if } \alpha>\beta \text { and } 1+\beta<2 \alpha \\
o_{p}\left(T \delta_{T}^{2\left(T_{c}-T_{e}\right)}\right) & f \alpha>\beta \text { and } 1+\beta>2 \alpha \\
o_{p}\left(T \delta_{T}^{2\left(T_{c}-T_{e}\right)}\right) & \text { if } \alpha<\beta \text { and } 1+\alpha>2 \beta \\
-T^{2 \beta-\alpha} \delta_{T}^{2\left(T_{c}-T_{e}\right)} c_{1} \frac{f_{c}-f_{e}}{f_{w} c_{2}^{2}} B\left(f_{e}\right)^{2} & \text { if } \alpha<\beta \text { and } 1+\alpha<2 \beta
\end{array}\right.
$$

(3) For $\tau_{1} \in N_{1}$ and $\tau_{2} \in N_{0}$,

$$
\sum_{j=\tau_{1}}^{\tau_{2}} \tilde{X}_{j-1}^{*}\left(\tilde{X}_{j}^{*}-\tilde{X}_{j-1}^{*}\right) \sim_{a}\left\{\begin{array}{cl}
T^{2 \alpha-\beta} \delta_{T}^{2\left(T_{c}-T_{e}\right)} c_{2} \frac{f_{2}-f_{c}}{f_{w} c_{1}^{2}} B\left(f_{e}\right)^{2} & \text { if } \alpha>\beta \text { and } 1+\beta<2 \alpha \\
o_{p}\left(T \delta_{T}^{2\left(T_{c}-T_{e}\right)}\right) & f \alpha>\beta \text { and } 1+\beta>2 \alpha \\
o_{p}\left(T \delta_{T}^{2\left(T_{c}-T_{e}\right)}\right) & \text { if } \alpha<\beta \text { and } 1+\alpha>2 \beta \\
-T^{2 \beta-\alpha} \delta_{T}^{2\left(T_{c}-T_{e}\right)} c_{1} \frac{f_{c}-f_{e}}{f_{w} c_{2}^{2}} B\left(f_{e}\right)^{2} & \text { if } \alpha<\beta \text { and } 1+\alpha<2 \beta
\end{array} .\right.
$$

(4) For $\tau_{1} \in C$ and $\tau_{2} \in B$,

$$
\sum_{j=\tau_{1}}^{\tau_{2}} \tilde{X}_{j-1}^{*}\left(\tilde{X}_{j}^{*}-\tilde{X}_{j-1}^{*}\right) \sim_{a}\left\{\begin{array}{cl}
T^{2 \alpha-\beta} \delta_{T}^{2\left(T_{c}-T_{e}\right)} c_{2} \frac{f_{2}-f_{c}}{f_{w} c_{1}^{2}} B\left(f_{e}\right)^{2} & \text { if } \alpha>\beta \text { and } 1+\beta<2 \alpha \\
o_{p}\left(T \delta_{T}^{2\left(T_{c}-T_{e}\right)}\right) & f \alpha>\beta \text { and } 1+\beta>2 \alpha \\
o_{p}\left(T \delta_{T}^{2\left(T_{c}-T_{e}\right)}\right) & \text { if } \alpha<\beta \text { and } 1+\alpha>2 \beta \\
-T^{2 \beta-\alpha} \delta_{T}^{2\left(T_{c}-T_{e}\right)} c_{1} \frac{f_{c}-f_{e}}{f_{w} c_{2}^{2}} B\left(f_{e}\right)^{2} & \text { if } \alpha<\beta \text { and } 1+\alpha<2 \beta
\end{array} .\right.
$$

(5) For $\tau_{1} \in N_{1}$ and $\tau_{2} \in B$,

$$
\sum_{j=\tau_{1}}^{\tau_{2}} \tilde{X}_{j-1}^{*}\left(\tilde{X}_{j}^{*}-\tilde{X}_{j-1}^{*}\right) \sim_{a} \begin{cases}T^{2 \alpha-\beta} \delta_{T}^{2\left(T_{c}-T_{e}\right)} \frac{f_{2}-f_{r}}{f_{w} c_{2}} B\left(f_{e}\right)^{2} & \text { if } \alpha>\beta \text { and } 1+\beta<2 \alpha \\ -T \delta_{T}^{2\left(T_{c}-T_{e}\right)} \frac{1}{2} B\left(f_{e}\right)^{2} & f \alpha>\beta \text { and } 1+\beta>2 \alpha \\ -T \delta_{T}^{2\left(T_{c}-T_{e}\right)} \frac{1}{2} B\left(f_{e}\right)^{2} & \text { if } \alpha<\beta \text { and } 1+\alpha>2 \beta \\ -T^{2 \beta-\alpha} \delta_{T}^{2\left(T_{c}-T_{e}\right)} c_{1} \frac{f_{c}-f_{1}}{f_{w} c_{2}^{2}} B\left(f_{e}\right)^{2} & \text { if } \alpha<\beta \text { and } 1+\alpha<2 \beta\end{cases}
$$

(6) For $\tau_{1} \in N_{1}$ and $\tau_{2} \in C$,

$$
\sum_{j=\tau_{1}}^{\tau_{2}} \tilde{X}_{j-1}^{*}\left(\tilde{X}_{j}^{*}-\tilde{X}_{j-1}^{*}\right) \sim_{a}\left\{\begin{array}{ll}
T^{2-\beta} c_{2} \frac{\left(f_{r}-f_{1}\right)\left(f_{2}-f_{r}\right)^{2}}{f_{w}^{2}}\left[\int_{f_{r}}^{f_{2}}\left[B(s)-B\left(f_{r}\right)\right] d s\right]^{2} & \text { if } \alpha>\beta \\
T \delta_{T}^{2\left(T_{c}-T_{e}\right)} \gamma_{T}^{2\left(T_{1}-T_{c}\right)} \frac{1}{2} B\left(f_{e}\right)^{2} & \text { if } \alpha<\beta
\end{array} .\right.
$$

\section{B.3.1 Test asymptotics}

The fitted regression model for the recursive unit root tests is

$$
X_{t}^{*}=\hat{\mu}_{g_{1}, g_{2}}+\hat{\rho}_{g_{1}, g_{2}} X_{t-1}^{*}+\hat{v}_{t}
$$

where the intercept $\hat{\mu}_{g_{1}, g_{2}}$ and slope coefficient $\hat{\rho}_{g_{1}, g_{2}}$ are obtained using data over the subperiod $\left[g_{1}, g_{2}\right]$. 
Remark 5 Based on Lemma B.10 and Lemma B.12, we can obtain limit forms of $\hat{\rho}_{g_{1}, g_{2}}-\gamma_{T}^{-1}$ using

$$
\hat{\rho}_{g_{1}, g_{2}}-\gamma_{T}^{-1}=\frac{\sum_{j=\tau_{1}}^{\tau_{2}} \tilde{X}_{j-1}^{*}\left(\tilde{X}_{j}^{*}-\gamma_{T}^{-1} \tilde{X}_{j-1}^{*}\right)}{\sum_{j=\tau_{1}}^{\tau_{2}} \tilde{X}_{j-1}^{* 2}} .
$$

When $\tau_{1} \in B$ and $\tau_{2} \in N_{0}$,

$$
\hat{\rho}_{g_{1, g_{2}}}-\gamma_{T}^{-1} \sim_{a}\left\{\begin{array}{ll}
-c_{2} T^{-\beta} & \text { if } \alpha>\beta \\
-c_{1} T^{-\alpha} & \text { if } \alpha<\beta
\end{array} .\right.
$$

when $\tau_{1} \in N_{1}$ and $\tau_{2} \in C$,

$$
\hat{\rho}_{g_{1}, g_{2}}-\gamma_{T}^{-1} \sim_{a}\left\{\begin{array}{lc}
-T^{-\beta} c_{2} \frac{\left\{\int_{f_{r}}^{f_{2}}\left[B(s)-B\left(f_{r}\right)\right]^{2} d s+\frac{\left(f_{2}-f_{r}\right)\left(f_{2}-f_{r}-2 f_{w}\right)}{f_{w}^{2}}\left[\int_{f_{r}}^{f_{2}}\left[B(s)-B\left(f_{r}\right)\right] d s\right]^{2}\right\}}{\left\{\int_{f_{r}}^{f_{2}}\left[B(s)-B\left(f_{r}\right)\right]^{2} d s-\frac{f_{2}-f_{r}}{f_{w}}\left[\int_{f_{r}}^{f_{2}}\left[B(s)-B\left(f_{r}\right)\right] d s\right]^{2}\right\}} & \text { if } \alpha>\beta \\
-2 T^{-1} \frac{f_{2}-f_{r}}{f_{w}} & \text { if } \alpha<\beta
\end{array}\right.
$$

for all other cases, we have

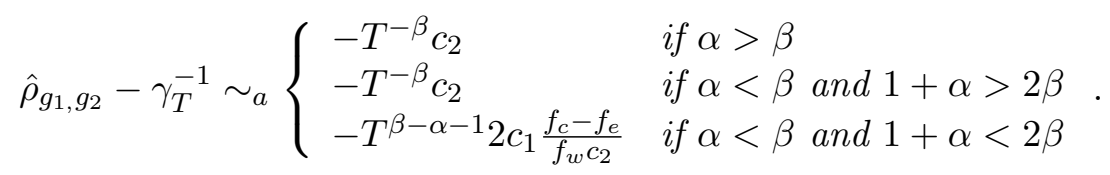

Remark 6 Based on Lemma B.10 and Lemma B.13, we can obtain limit forms of $\hat{\rho}_{g_{1}, g_{2}}-\delta_{T}^{-1}$ using

$$
\hat{\rho}_{g_{1}, g_{2}}-\delta_{T}^{-1}=\frac{\sum_{j=\tau_{1}}^{\tau_{2}} \tilde{X}_{j-1}^{*}\left(X_{j}^{*}-\delta_{T}^{-1} X_{j-1}^{*}\right)}{\sum_{j=\tau_{1}}^{\tau_{2}} \tilde{X}_{j-1}^{* 2}} .
$$

When $\tau_{1} \in B$ and $\tau_{2} \in N_{0}$,

$$
\hat{\rho}_{g_{1}, g_{2}}-\delta_{T}^{-1} \sim_{a} \frac{1}{T} 2 c_{1} \frac{f_{e}-f_{1}}{f_{w}}
$$

When $\tau_{1} \in C$ and $\tau_{2} \in N_{0}$,

$$
\hat{\rho}_{g_{1}, g_{2}}-\delta_{T}^{-1} \sim_{a}\left\{\begin{array}{ll}
T^{-\alpha} \frac{{ }_{1}\left\{\int_{f_{r}}^{f_{2}}\left[B(s)-B\left(f_{r}\right)\right]^{2} d s+\frac{\left(f_{2}-f_{r}\right)\left(f_{2}-f_{r}-2 f_{w}\right)}{f_{w}^{2}}\left[\int_{f_{r}}^{f_{2}}\left[B(s)-B\left(f_{r}\right)\right] d s\right]^{2}\right\}}{\int^{2} f_{2}\left[B(s)-B\left(f_{r}\right)\right]^{2} d s-\frac{f_{2}-f_{r}}{f_{w}}\left[\int_{f_{r}}^{f_{2}}\left[B(s)-B\left(f_{r}\right)\right] d s\right]^{2}} & \text { if } \alpha>\beta \\
2 T^{\beta-\alpha-1} c_{1} \frac{f_{2}-f_{r}}{f_{w} c_{2}} & \text { if } \alpha<\beta
\end{array} ;\right.
$$

For all other cases

$$
\hat{\rho}_{g_{1, g_{2}}}-\delta_{T}^{-1} \sim_{a}\left\{\begin{array}{ll}
T^{\alpha-\beta-1} K & \text { if } \alpha>\beta \text { and } 1+\beta<2 \alpha \\
c_{1} T^{-\alpha} & \text { if } \alpha>\beta \text { and } 1+\beta>2 \alpha \\
c_{1} T^{-\alpha} & \text { if } \alpha<\beta
\end{array} ;\right.
$$

where $K$ is a constant which equals $2 c_{1} c_{2} \frac{f_{r}-f_{c}}{f_{w} c_{1}^{2}}$ when $\tau_{1} \in N_{1}$ and $\tau_{2} \in N_{0}$ and when $\tau_{1} \in N_{1}$ and $\tau_{2} \in B$ and equals $2 c_{1} c_{2} \frac{f_{2}-f_{c}}{f_{w} c_{1}^{2}}$ when $\tau_{1} \in C$ and $\tau_{2} \in B$ and when $\tau_{1} \in C$ and $\tau_{2} \in N_{0}$. 
Remark 7 Based on Lemma B.10 and Lemma B.13, we can obtain limit forms of $\hat{\rho}_{g_{1}, g_{2}}-1$ using

$$
\hat{\rho}_{g_{1}, g_{2}}-1=\frac{\sum_{j=\tau_{1}}^{\tau_{2}} \tilde{X}_{j-1}^{*}\left(X_{j}^{*}-X_{j-1}^{*}\right)}{\sum_{j=\tau_{1}}^{\tau_{2}} \tilde{X}_{j-1}^{* 2}} .
$$

When $\tau_{1} \in B$ and $\tau_{2} \in N_{0}$,

$$
\hat{\rho}_{g_{1, g_{2}}}-1 \sim_{a} \frac{c_{1}}{T^{\alpha}}
$$

when $\tau_{1} \in N_{1}$ and $\tau_{2} \in C$,

$$
\hat{\rho}_{g_{1}, g_{2}}-1^{\sim} \begin{cases}T^{-\beta} c_{2} \frac{\frac{\left(f_{r}-f_{1}\right)\left(f_{2}-f_{r}\right)}{f_{w}^{2}}\left[\int_{f_{r}}^{f_{2}}\left[B(s)-B\left(f_{r}\right)\right] d s\right]^{2}}{\int_{f_{r}}^{f_{2}}\left[B(s)-B\left(f_{r}\right)\right]^{2} d s-\frac{f_{2}-f_{r}}{f_{w}}\left[\int_{f_{r}}^{f_{2}}\left[B(s)-B\left(f_{r}\right)\right] d s\right]^{2}} & \text { if } \alpha>\beta \\ T^{-\beta} c_{2} & \text { if } \alpha<\beta\end{cases}
$$

when $\tau_{1} \in N_{1}$ and $\tau_{2} \in B$,

$$
\hat{\rho}_{g_{1}, g_{2}}-1 \sim_{a} \begin{cases}T^{\alpha-\beta-1} 2 c_{1} \frac{f_{2}-f_{r}}{f_{w} c_{2}} & \text { if } \alpha>\beta \text { and } 1+\beta<2 \alpha \\ -c_{1} T^{-\alpha} & \text { f } \alpha>\beta \text { and } 1+\beta>2 \alpha \\ -T^{-\beta} c_{2} & \text { if } \alpha<\beta \text { and } 1+\alpha>2 \beta \\ -T^{\beta-\alpha-1} 2 c_{1} \frac{f_{c}-f_{1}}{f_{w} c_{2}} & \text { if } \alpha<\beta \text { and } 1+\alpha<2 \beta\end{cases}
$$

for all other cases, we have

$$
\hat{\rho}_{g_{1, g_{2}}}-1 \sim_{a} \begin{cases}T^{\alpha-\beta-1} 2 c_{1} \frac{f_{2}-f_{r}}{f_{w} c_{2}} & \text { if } \alpha>\beta \text { and } 1+\beta<2 \alpha \\ o_{p}\left(T^{-\alpha}\right) & f \alpha>\beta \text { and } 1+\beta>2 \alpha \\ o_{p}\left(T^{-\beta}\right) & \text { if } \alpha<\beta \text { and } 1+\alpha>2 \beta \\ -T^{\beta-\alpha-1} 2 c_{1} \frac{f_{c}-f_{1}}{f_{w} c_{2}} & \text { if } \alpha<\beta \text { and } 1+\alpha<2 \beta\end{cases}
$$

Based on the above three remarks, one can see that the quantity $\hat{\rho}_{g_{1}, g_{2}}-\gamma_{T}^{-1}$ diverges to negative infinity and the quantity $\hat{\rho}_{g_{1}, g_{2}}-\delta_{T}^{-1}$ diverges to positive infinity. In other words, the estimated value of $\hat{\rho}_{g_{1}, g_{2}}$ is bounded by $\gamma_{T}^{-1}$ and $\delta_{T}^{-1}$. Furthermore, the quantity $\hat{\rho}_{g_{1}, g_{2}}-1$ diverges to positive infinity when $\tau_{1} \in B$ and $\tau_{2} \in N_{0}$ and when $\tau_{1} \in N_{1}$ and $\tau_{2} \in C$. For all other cases, the quantity $\hat{\rho}_{g_{1}, g_{2}}-1$ diverges to positive infinity when bubble collapsing speed is much faster than expansion rate (i.e. $1+\beta<2 \alpha$ ) and to negative infinity otherwise.

Lemma B.15 To obtain the asymptotic behaviors of the Dickey-Fuller t-statistic, we first obtain the equation standard error of the regression over $\left[T_{1}, T_{2}\right]$ is

$$
\hat{\sigma}_{g_{1} g_{2}}=\left\{\tau_{w}^{-1} \sum_{j=\tau_{1}}^{\tau_{2}}\left(\tilde{X}_{j}^{*}-\hat{\rho}_{g_{1}, g_{2}} \tilde{X}_{j-1}^{*}\right)^{2}\right\}^{1 / 2} .
$$

(1) When $\tau_{1} \in B$ and $\tau_{2} \in N_{0}$,

$$
\hat{\sigma}_{g_{1} g_{2}}^{2}=O_{p}\left(T^{-1} \delta_{T}^{2\left(T_{2}-T_{e}\right)}\right)
$$


(2) When $\tau_{1} \in C$ and $\tau_{2} \in N_{0}$,

$$
\hat{\sigma}_{g_{1}, g_{2}}^{2}= \begin{cases}O_{p}\left(T^{2 \alpha-2 \beta-1} \delta_{T}^{2\left(T_{c}-T_{e}\right)}\right) & \text { if } \alpha>\beta \text { and } 1+\beta<2 \alpha \\ O_{p}\left(T^{-\beta} \delta_{T}^{2\left(T_{c}-T_{e}\right)}\right) & \text { f } \alpha>\beta \text { and } 1+\beta>2 \alpha \\ O_{p}\left(T^{-\alpha} \delta_{T}^{2\left(T_{c}-T_{e}\right)}\right) & \text { if } \alpha<\beta \text { and } 1+\alpha>2 \beta \\ O_{p}\left(T^{2 \beta-2 \alpha-1} \delta_{T}^{2\left(T_{c}-T_{e}\right)}\right) & \text { if } \alpha<\beta \text { and } 1+\alpha<2 \beta\end{cases}
$$

(3) When $\tau_{1} \in N_{1}$ and $\tau_{2} \in N_{0}$,

$$
\hat{\sigma}_{g_{1}, g_{2}}^{2}= \begin{cases}O_{p}\left(T^{2 \alpha-2 \beta-1} \delta_{T}^{2\left(T_{c}-T_{e}\right)}\right) & \text { if } \alpha>\beta \text { and } 1+\beta<2 \alpha \\ O_{p}\left(T^{-\beta} \delta_{T}^{2\left(T_{c}-T_{e}\right)}\right) & f \alpha>\beta \text { and } 1+\beta>2 \alpha \\ O_{p}\left(T^{-\alpha} \delta_{T}^{2\left(T_{c}-T_{e}\right)}\right) & \text { if } \alpha<\beta \text { and } 1+\alpha>2 \beta \\ O_{p}\left(T^{2 \beta-2 \alpha-1} \delta_{T}^{2\left(T_{c}-T_{e}\right)}\right) & \text { if } \alpha<\beta \text { and } 1+\alpha<2 \beta\end{cases}
$$

(4) When $\tau_{1} \in C$ and $\tau_{2} \in B$,

$$
\hat{\sigma}_{g_{1}, g_{2}}^{2}= \begin{cases}O_{p}\left(T^{2 \alpha-2 \beta-1} \delta_{T}^{2\left(T_{c}-T_{e}\right)}\right) & \text { if } \alpha>\beta \text { and } 1+\beta<2 \alpha \\ O_{p}\left(T^{-\beta} \delta_{T}^{2\left(T_{c}-T_{e}\right)}\right) & \text { f } \alpha>\beta \text { and } 1+\beta>2 \alpha \\ O_{p}\left(T^{-\alpha} \delta_{T}^{2\left(T_{c}-T_{e}\right)}\right) & \text { if } \alpha<\beta \text { and } 1+\alpha>2 \beta \\ O_{p}\left(T^{2 \beta-2 \alpha-1} \delta_{T}^{2\left(T_{c}-T_{e}\right)}\right) & \text { if } \alpha<\beta \text { and } 1+\alpha<2 \beta\end{cases}
$$

(5) When $\tau_{1} \in N_{1}$ and $\tau_{2} \in B$,

$$
\hat{\sigma}_{g_{1}, g_{2}}^{2}= \begin{cases}O_{p}\left(T^{2 \alpha-2 \beta-1} \delta_{T}^{2\left(T_{c}-T_{e}\right)}\right) & \text { if } \alpha>\beta \text { and } 1+\beta<2 \alpha \\ O_{p}\left(T^{-\beta} \delta_{T}^{2\left(T_{c}-T_{e}\right)}\right) & \text { f } \alpha>\beta \text { and } 1+\beta>2 \alpha \\ O_{p}\left(T^{-\alpha} \delta_{T}^{2\left(T_{c}-T_{e}\right)}\right) & \text { if } \alpha<\beta \text { and } 1+\alpha>2 \beta \\ O_{p}\left(T^{2 \beta-2 \alpha-1} \delta_{T}^{2\left(T_{c}-T_{e}\right)}\right) & \text { if } \alpha<\beta \text { and } 1+\alpha<2 \beta\end{cases}
$$

(6) When $\tau_{1} \in N_{1}$ and $\tau_{2} \in C$,

$$
\hat{\sigma}_{g_{1}, g_{2}}^{2}= \begin{cases}O_{p}\left(T^{1-2 \beta}\right) & \text { if } \alpha>\beta \\ O_{p}\left(T^{-1} \delta_{T}^{2\left(T_{c}-T_{e}\right)} \gamma_{T}^{2\left(T_{1}-T_{c}\right)}\right) & \text { if } \alpha<\beta\end{cases}
$$

The asymptotic distribution of the Dickey-Fuller t statistic

$$
D F_{g_{1}, g_{2}}^{t}=\left(\frac{\sum_{j=\tau_{1}}^{\tau_{2}} \tilde{X}_{j-1}^{* 2}}{\hat{\sigma}^{2}}\right)^{1 / 2}\left(\hat{\rho}_{g_{1}, g_{2}}-1\right)
$$

can be calculated as follows. Notice that the sign of the DF statistic is determined by the quantity $\hat{\rho}_{g_{1}, g_{2}}-1$. 
Remark 8 When $\tau_{1} \in B$ and $\tau_{2} \in N_{0}$,

$$
D F_{g_{1}, g_{2}}^{t}=\left(\frac{\sum_{j=\tau_{1}}^{\tau_{2}} \tilde{X}_{j-1}^{* 2}}{\hat{\sigma}^{2}}\right)^{1 / 2}\left(\hat{\rho}_{g_{1}, g_{2}}-1\right)=O_{p}\left(T^{1-\alpha / 2}\right) \rightarrow+\infty
$$

when $\tau_{1} \in N_{1}$ and $\tau_{2} \in C$,

$$
D F_{g_{1}, g_{2}}^{t}=\left(\frac{\sum_{j=\tau_{1}}^{\tau_{2}} \tilde{X}_{j-1}^{* 2}}{\hat{\sigma}^{2}}\right)^{1 / 2}\left(\hat{\rho}_{g_{1}, g_{2}}-1\right)=\left\{\begin{array}{ll}
O_{p}\left(T^{1 / 2}\right) \rightarrow+\infty & \text { if } \alpha>\beta \\
O_{p}\left(T^{1-\beta / 2}\right) \rightarrow+\infty & \text { if } \alpha<\beta
\end{array} .\right.
$$

when $\tau_{1} \in N_{1}$ and $\tau_{2} \in B$,

$$
D F_{g_{1}, g_{2}}^{t}=\left(\frac{\sum_{j=\tau_{1}}^{\tau_{2}} \tilde{X}_{j-1}^{* 2}}{\hat{\sigma}^{2}}\right)^{1 / 2}\left(\hat{\rho}_{g_{1}, g_{2}}-1\right) \sim_{a}\left\{\begin{array}{ll}
O_{p}\left(T^{\alpha / 2}\right) \rightarrow+\infty & \text { if } \alpha>\beta \text { and } 1+\beta<2 \alpha \\
O_{p}\left(T^{(1-\alpha+\beta) / 2}\right) \rightarrow-\infty & \text { if } \alpha>\beta \text { and } 1+\beta>2 \alpha \\
O_{p}\left(T^{(1-\beta+\alpha) / 2}\right) \rightarrow-\infty & \text { if } \alpha<\beta \text { and } 1+\alpha>2 \beta \\
O_{p}\left(T^{\beta / 2}\right) \rightarrow-\infty & \text { if } \alpha<\beta \text { and } 1+\alpha<2 \beta
\end{array} .\right.
$$

for all other cases

$$
D F_{g_{1}, g_{2}}^{t}=\left(\frac{\sum_{j=\tau_{1}}^{\tau_{2}} \tilde{X}_{j-1}^{* 2}}{\hat{\sigma}^{2}}\right)^{1 / 2}\left(\hat{\rho}_{g_{1}, g_{2}}-1\right) \sim_{a}\left\{\begin{array}{ll}
O_{p}\left(T^{\alpha / 2}\right) \rightarrow+\infty & \text { if } \alpha>\beta \text { and } 1+\beta<2 \alpha \\
o_{p}\left(T^{(1-\alpha+\beta) / 2}\right) & \text { if } \alpha>\beta \text { and } 1+\beta>2 \alpha \\
o_{p}\left(T^{(1-\beta+\alpha) / 2}\right) & \text { if } \alpha<\beta \text { and } 1+\alpha>2 \beta \\
O_{p}\left(T^{\beta / 2}\right) \rightarrow-\infty & \text { if } \alpha<\beta \text { and } 1+\alpha<2 \beta
\end{array} .\right.
$$

Given that $g_{2}=g$ and $g_{1} \in\left[0, g-g_{0}\right]$, the asymptotic behavior of the backward sup DF statistic under the alternative hypothesis are:

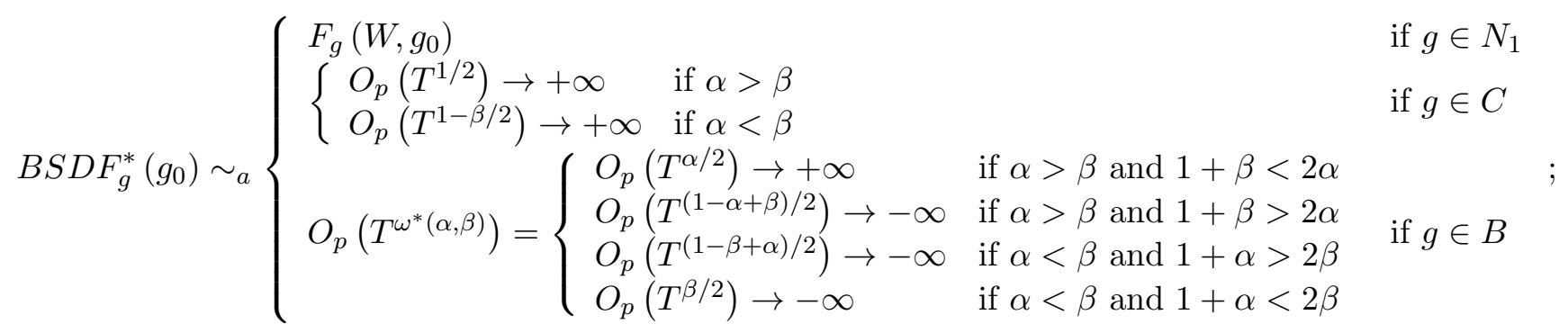

This proves Theorem 3.4. Following the standard probability arguments (see PSY), we deduce that $\operatorname{Pr}\left\{\left|\hat{g}_{e}-g_{e}\right|>\eta\right\} \rightarrow 0$ and $\operatorname{Pr}\left\{\left|\hat{g}_{c}-g_{c}\right|>\gamma\right\} \rightarrow 0$ for any $\eta, \gamma>0$ as $T \rightarrow \infty$, provided that

$$
\left\{\begin{array}{ll}
\frac{T^{\alpha / 2}}{s c v^{*}\left(\beta_{T}\right)}+\frac{s c v^{*}\left(\beta_{T}\right)}{T^{1 / 2}} \rightarrow 0 & \text { if } \alpha>\beta \text { and } 1+\beta<2 \alpha \\
\frac{T^{(1-\alpha+\beta) / 2}}{s c v^{*}\left(\beta_{T}\right)}+\frac{s c v^{*}\left(\beta_{T}\right)}{T^{1 / 2}} \rightarrow 0 & \text { if } \alpha>\beta \text { and } 1+\beta>2 \alpha \\
\frac{T^{(1-\beta+\alpha) / 2}}{s c v^{*}\left(\beta_{T}\right)}+\frac{s c v^{*}\left(\beta_{T}\right)}{T^{1-\beta / 2}} \rightarrow 0 & \text { if } \alpha<\beta \text { and } 1+\alpha>2 \beta \\
\frac{T^{\beta / 2}}{s c v^{*}\left(\beta_{T}\right)}+\frac{s v^{*}\left(\beta_{T}\right)}{T^{1-\beta / 2}} \rightarrow 0 & \text { if } \alpha<\beta \text { and } 1+\alpha<2 \beta
\end{array} .\right.
$$

Therefore, $\hat{f}_{r}=1-\hat{g}_{e}$ and $\hat{f}_{c}=1-\hat{g}_{c}$ are consistent estimators of $f_{r}$ and $f_{c}$. This proves Theorem 3.5 . 